\title{
1 The role of maternal pioneer factors in predefining first 2 zygotic responses to inductive signals
}

\author{
3 George E. Gentsch ${ }^{1}$, Thomas Spruce ${ }^{2}$, Nick D. L. Owens ${ }^{3}$ \& James C. Smith ${ }^{1}$ \\ $4 \quad{ }^{1}$ Developmental Biology Laboratory, The Francis Crick Institute, London NW1 1AT, UK \\ $5 \quad{ }^{2}$ Centre for Genomic Regulation, Barcelona Institute for Science and Technology, 08003 Barcelona, Spain \\ $6 \quad{ }^{3}$ Department of Stem Cell and Developmental Biology, Pasteur Institute, 75015 Paris, France \\ 7 Correspondence should be addressed to G.E.G (george.gentsch@crick.ac.uk) or J.C.S (jim.smith@crick.ac.uk)
}

\section{ABSTRACT}

9 Embryonic development yields many different cell types in response to just a few families of inductive signals.

10 The property of a signal-receiving cell that determines how it responds to such signals, including the activation 11 of cell type-specific genes, is known as its competence. Here, we show how maternal factors modify chromatin to specify initial competence in the frog Xenopus tropicalis. We identified the earliest engaged regulatory DNA sequences, and inferred from them critical activators of the zygotic genome. Of these, we showed that the pioneering activity of the maternal pluripotency factors Pou5f3 and Sox 3 predefines competence for germ layer formation by extensively remodeling compacted chromatin before the onset of signaling. The remodeling includes the opening and marking of thousands of regulatory elements, extensive chromatin looping, and the co-recruitment of signal-mediating transcription factors. Our work identifies significant developmental principles that inform our understanding of how pluripotent stem cells interpret inductive signals.

\section{INTRODUCTION}

The specification of different cell types during embryonic development is achieved through the repeated use of a small number of highly conserved intercellular signals. The property of a cell that defines the way it responds to such signals (if it responds at all) is known as its competence ${ }^{1}$. Classical experiments with amphibian embryos show that competence is regulated in both space and time. For example, head ectoderm of the tailbud embryo responds to the underlying optic vesicle by forming a lens, while other surface ectoderm is unable to do $\mathrm{so}^{2}$. Similarly, on a temporal scale, naïve pluripotent (animal cap) tissue of the early Xenopus embryo loses the ability to form muscle in response to Nodal signaling by the mid-gastrula stage ${ }^{3}$.

How is competence regulated at the molecular level? A simple mechanism would involve the acquisition or loss of components of a signal transduction pathway, but this fails to explain differences in context-dependent responses to the same signals. In addition, although there are some tissue-specific signal receptor isoforms, transduction to the nucleus is limited to just a few signal mediators with transcriptional activity such as $\beta$-catenin (canonical Wnt signaling), Smad2 (Nodal signaling) and Smad1 (BMP signaling). Thus, a more plausible and common way in which tissue-specific competence might arise is through the recruitment of these signal mediators to different gene regulatory sites which in turn would drive the specification of different cell types.

A series of experiments indicates that cell lineage determinants such as sequence-specific transcription factors (TFs) may play a role in determining competence. For lens induction, the responding cells of the head ectoderm depend on the presence of the eye-regulating TF Pax6 ${ }^{4}$. However, although we know that certain TFs can act as competence factors, these TFs have not been 
identified in a systematic fashion, and their modes of action remain largely unknown. Our understanding of how chromatin interprets inductive signals is especially important because the generation of therapeutically relevant cells like insulin-producing pancreatic $\beta$-cells frequently relies on the deployment of signal modulators at different stages of cell differentiation in vitro ${ }^{5}$. Moreover, the initiation and progression of tumors is often associated with a change in their competence which, in some instances, is correlated with the erroneous re-activation of embryonic TFs stimulating excessive cell proliferation ${ }^{6}$.

In an effort to analyze systematically the molecular basis of competence, we chose, for the following reasons, to analyze the first inductive signaling events in the embryo of Xenopus tropicalis. First, like most multicellular organisms, X. tropicalis begins development with a transcriptionally silent genome (Fig. 1a). After fertilization, twelve rapid cleavages convert the egg into a mid-blastula embryo with little transcription and little diversification among its 4,096 cells ${ }^{7}$. Both the low levels of transcription and the cellular homogeneity assist in the interpretation of whole-embryo and loss-of-function chromatin profiling. Second, TFs involved in zygotic (or embryonic) genome activation (ZGA) are likely to occupy top-level positions within gene regulatory networks, and their binding should thus correlate strongly with gene target expression ${ }^{8}$. This makes them more amenable to study. Third, based on the nuclear accumulation of signal mediators ${ }^{9}$ (Fig. 1a), we know a great deal about where and when inductive signaling occurs during ZGA. And finally, in vitro fertilization yields thousands of synchronously developing embryos, which greatly facilitates temporal chromatin profiling.

Taking advantage of these features of $X$. tropicalis, we have used transcriptional, translational and multi-level chromatin profiling to help identify the earliest regulatory DNA sequences (hereafter called putative cis-regulatory modules, pCRMs). From these we infer the critical maternal activators of the zygotic genome as potential competence factors. We next compared the DNA binding of several maternal/zygotic TFs and signal mediators across the mid-blastula transition (MBT) to reveal the effect of TF co-expression on chromatin recruitment in vivo. Finally, we have demonstrated how maternal TFs of the pluripotency network structure the chromatin landscape, which in turn determines the signal-mediated regionalization of gene activity and the specification of the three germ layers: ectoderm, mesoderm and endoderm.

\section{RESULTS}

\section{DNA motifs at pCRMs correlate with the affinities of frequently translated TFs during ZGA}

In an effort to understand how early chromatin dynamics influence the recruitment of signal mediators to the genome (Fig. 1b), we first identified $\sim 27,000 \mathrm{pCRMs}$ from the 32-cell to the late gastrula stage by mapping focal RNA polymerase II (RNAPII) depositions on a genome-wide scale by means of ChIP-Seq (Fig. 1c and Supplementary Tables 1 and 2). RNAPII has no DNA sequence preference and its chromatin engagement is a reliable and objective indicator of pCRM usage ${ }^{10}$. The number of RNAPIIengaged (RNAPII ${ }^{+}$) pCRMs increased from $\sim 650$ at the 32-cell stage to $>10,000$ at the 1,024-cell and later developmental stages (Supplementary Fig. 1a). The largest changes to RNAPII ${ }^{+}$pCRMs, as calculated by pairwise Spearman correlations, were detected between the 1,024-cell stage and the MBT (Fig. 1d), with most pCRMs being engaged only transiently before MBT (e.g., 6,145 from the 128-cell to the 1,024-cell stage) and more persistently after MBT (Supplementary Fig. 1a). The analysis of enriched DNA motifs among RNAPII ${ }^{+}$pCRMs suggests that pre-MBT recruitment is predominantly directed by members of the FOXH, POU, SOX and T domain TF families (Fig. 1e and Supplementary Fig. 1b).

The discovery of RNAPII ${ }^{+}$pCRMs is difficult in promoters and gene bodies where extended RNAPII depositions associated with transcript elongation might hamper the correct detection of RNAPII ${ }^{+}$pCRMs. MBT-staged pCRMs were therefore further characterized for chromatin accessibility and for the enhancer-associated histone mark H3K4me1. In our hands, the high yolk content in early $X$. tropicalis embryos made it impossible to use transposition ${ }^{11}$ to probe chromatin accessibility. Instead, we used an approach involving DNase I mediated digestion followed by deep sequencing (DNase-Seq), in which we selected small accessible fragments of DNA (see Methods and Supplementary Fig. 1c for exemplar comparison with other chromatin features). 
pCRMs (compared with $\sim 16 \%$ and $\sim 38 \%$ of pCRMs detected by RNAPII peak calling) were found in promoters and gene bodies, respectively (Supplementary Fig. 1e).

Enriched DNA motifs among accessible/RNAPII ${ }^{+} / \mathrm{H} 3 \mathrm{~K}_{4} \mathrm{me}^{+}$pCRMs were then correlated with maternally inherited and translated sequence-specific factors identified by egg-staged mass spectrometry ${ }^{12}$ and pre-MBT ribosome footprinting ${ }^{13}$ to identify members of the TF families that may play a role in the ZGA (Fig. 2a, Supplementary Fig. 2a and Supplementary Table 4). It proved that the binding preferences of the most frequently translated maternal TFs and signal mediators matched the most significantly enriched DNA motifs (Fig. 2b). These were POU-SOX (Pou5f3-Sox3 heterodimer), Krüppel-like zinc finger (ZF; Sp1 and several Klf), POU (Pou5f3), SOX (Sox3), bZIP (Max), FOXH (Foxh1), ETS (Ets2), NFY (NFYa/b/c), SMAD (Smad1/2), $\mathrm{T}$ (mVegT, a maternal VegT isoform), TCF (Tcf/ß-catenin), basic helix-span-helix domain (bHSH; Tfap2), and OTX (Otx1). Interestingly, the enrichment level of these motifs was independent of pCRM sequence conservation (based on phastCons scoring) detected among vertebrates (Figs. 1f and 2b).

Of the most frequently translated TFs with cognate pCRM-enriched DNA recognition motifs, we selected Pou5f3, Sox3 and $\mathrm{mVegT}$ as potential competence factors of canonical Wnt, Nodal and BMP signaling. Sox3 and presumably also Pou5f3 (based on the spatial distribution of its maternal transcripts Pouff3.2 and Pou $5 f 3.3^{14}$ ) are detected ubiquitously (Fig. 2c), while mVegT is restricted to the vegetal hemisphere ${ }^{15}$. The zygotic isoform of VegT (zVegT) is expressed within the marginal zone (Fig. 2d and Supplementary Fig. 2e). With respect to signal mediators, nuclear $\beta$-catenin begins to accumulate weakly on the dorsal side of the embryo (first detected at the 32-cell stage ${ }^{16}$ ) before spreading more prominently around the upper lip of the forming blastopore after the $\mathrm{MBT}^{9}$ (Fig. 2d). Signal-induced nuclear accumulation of Smad1 and Smad2 is first detected around the MBT, with Smad1 being preferentially ventral and Smad2 being vegetal and within the marginal zone ${ }^{9,17}$ (Fig. 2d and Supplementary Fig. 2h). the MBT (Fig. 3a and Supplementary Table 1) to ask whether the recruitment of $\beta$-catenin, Smad1 and Smad2 might be influenced by co-expressed maternal TFs like Sox3, mVegT and Foxh $1{ }^{18}$ or vice versa (see Fig. 2c and Supplementary Fig. 2b$\mathbf{n}$ for ChIP antibody verification). As an outgroup control, we selected the binding profiles of several zygotic T-box TFs including Eomes (Eomesodermin), zVegT, Tbxt (Brachyury) ${ }^{19}$ and Tbx6, which collectively regulate the neuro-mesodermal cell lineage during and beyond gastrulation ${ }^{20}$. The profiled developmental stages and chromatin factors are color-coded as illustrated in Fig. 3a and ChIP-Seq peak call coordinates are listed in Supplementary Table 5.

Maternal and zygotic TFs and signal mediators shared many chromatin characteristics: (1) DNA occupancy levels followed a log-normal distribution with $<1,000$ RNAPII-transcribed genes receiving high and super-enhancer-like ${ }^{21}$ input (i.e. clusters of occupied pCRMs separated by $\leq 25 \mathrm{~kb}$ ). Similar distributions were also observed for chromatin accessibility and RNAPII engagement (see examples in Fig. 3a and Supplementary Fig. 3a,b and systematic analysis in Supplementary Fig. 4a). (2) From the 1,024-cell to the late gastrula stage, TF-bound super-enhancers were linked through the gene ontologies of nearby zygotic genes ( $\leq 5 \mathrm{~kb}$ ) to early embryonic processes including germ layer and body axis formation (Fig. 3b). (3) The binding sites of TFs and signal mediators were also frequently defined by RNAPII deposition (Fig. 3c). (4) Among all zygotic genes, promoterproximal regions were more consistently bound than other pCRMs (Supplementary Fig. 4 b).

Differences in DNA occupancy levels at different developmental stages, compared by pairwise Spearman correlations and principal component (PC) analysis, suggest that the recruitment to chromatin of signal mediators and TFs is driven both by their individual properties and by the developmental stage (Fig. 3d and Supplementary Fig. 5a). For example, chromatin recruitment of $\mathrm{mVegT}$ at stages of pluripotency resembled that of other sequence-specific factors at the same developmental stage but differed from the binding of its zygotic isoform and of related T-box TFs at later developmental stages (highlighted in Supplementary Fig. 5a). The importance of developmental stage in driving patterns of chromatin recruitment was further revealed by the changing DNA binding patterns of sequence-nonspecific RNAPII to pCRMs (Fig. 3e).

The identification of enriched DNA recognition motifs at occupied pCRMs confirmed known TF/signal mediator properties such as the sequence-specificity of their DNA binding domains, oligomerization tendencies and protein-protein interactions 
(Supplementary Fig. 5b). For example, in contrast to other T-box TFs, Tbxt recognizes palindromic T motifs due to its propensity to form homodimers ${ }^{22}$; and Smad2 chromatin recruitment is frequently associated with the FOXH motif because Smad2 interacts with Foxh $1^{23}$ (Supplementary Fig. 5b). Other DNA motifs such as the POU or POU-SOX motifs were consistently co-enriched from the 1,024-cell to the late gastrula stage in most binding profiles. This is indicative of the pluripotent state, a developmental context associated with co-expression of Pou5f (Oct4) and SoxB1 (e.g. Sox2 or Sox3) proteins as previously observed in vitro ${ }^{24}$ (Fig. 3g and Supplementary Fig. 5b).

The hierarchical clustering of DNA occupancy levels for the selected factors at different developmental stages revealed specific DNA motif combinations. For example, pCRMs showing 'unique' binding of mVegT (that is, binding that is not shared with the other profiled factors) show a high frequency of T, OTX and SOX motifs (Supplementary Fig. 6).

With respect to the chromatin recruitment of TFs versus signal mediators, we note that, first, Smads and/or $\beta$-catenin were frequently detected at Sox3, Foxh1 or VegT binding sites (top 2,000 peaks shown in Fig. 3f) and, second, Smad- and/or $\beta$-cateninbound pCRMs (top 2,000 peaks) were significantly enriched for SOX, POU-SOX, POU, FOXH and T motifs (red field \#1 in Fig. 3g) suggesting that corresponding TFs affect the recruitment of these signal mediators. The reverse was much less the case as shown by the low significance of SMAD and bHSH motif enrichments at TF-bound pCRMs (with the exception of Smad2interactor Foxh $1^{23}$ ) (blue field \#2 in Fig. 3g).

\section{TF co-expression drives the dynamics of chromatin recruitment}

To explore the suggested importance of TF co-expression on pCRM engagement, we ectopically expressed an HA-tagged version of the muscle determinant MyoD (MyoD-HA) in animal cap cells and profiled pCRMs for MyoD-HA as well as for endogenous Sox3 and RNAPII at the early gastrula stage (Fig. 4a and Supplementary Tables 1 and 6). MyoD was chosen because its canonical E-box recognition motif is normally not significantly enriched before or during gastrulation (green field \#3 in Fig. $\mathbf{3 g}$ and Supplementary Fig. 5b) so its effect on chromatin engagement ought to be clearly discernible, while Sox3 and RNAPII were selected because they are ubiquitously expressed and represent sequence-specific and nonspecific DNA binding factors, respectively. The ectopic expression elevated the Spearman correlations of Sox3 and RNAPII with MyoD-HA (Supplementary Fig. 7a) and shifted the first and second PC of Sox3 and RNAPII toward MyoD-HA (Fig. 4b) suggesting that MyoD-HA altered the binding of both Sox3 and RNAPII. Differential binding analysis on a genome-wide (heat map in Fig. 4c) and locus-specific (pileup track in Fig. 4d) scale confirms that MyoD-HA co-recruits endogenous Sox3 and RNAPII to its gene targets like actc1 and myll. The E-box motif of MyoD-HA emerged as a significantly enriched motif of Sox3 and RNAPII binding, while MyoDHA binding itself seemed to be influenced by endogenous TFs as judged by the developmental stage-characteristic enrichment of SOX, POU-SOX and FOX motifs at MyoD ${ }^{+}$pCRMs (Fig. 4e). However, this opportunistic recruitment to non-canonical binding sites, such as MyoD-HA to functional Sox3 gene targets (e.g. otx2 and sox2 activated by Sox3-HA), did not affect transcription in animal caps (Fig. $\mathbf{4 f - h})$.

157 The influence of TF co-expression on DNA occupancy was further substantiated by profiling chromatin for Sox 3 in different 158 anterior-posterior regions of the central nervous system (CNS) (Fig. 5a and Supplementary Tables $\mathbf{1}$ and 7). The analysis of enriched DNA motifs suggested that Sox3 binding was affected by differentially expressed homeodomain TFs such as orthodenticle homeobox (OTX) in the brain (head) and caudal homeobox (CDX) in the spinal cord (trunk, bud) (Fig. 5c and Supplementary Fig. 7c,d; see Fig. 5d for graphical illustration of TF co-expression at the posterior end of the embryo). This was particularly apparent in Sox3 binding to the colinear HoxD cluster defining anterior-posterior cell identity (Fig. 5b). Similar influences on chromatin engagement were observed within anterior and posterior mesoderm marked by Eomes in gastrula embryos and Tbxt/Tbx6 in gastrula and early tailbud embryos, respectively (Fig. 5c,d and Supplementary Fig. 7b,c). On a temporal scale, the influence of FOXH and POU motif-recognizing TFs in recruiting Sox3 and T-box TF was more pronounced early than late in development (Fig. 5c and Supplementary Figs. 5b and 7c). 
To ask whether signal-mediated ZGA requires maternal TFs, as suggested by the observed chromatin dynamics (Fig. 3), we next compared the effects of loss of maternal Sox3/PouV (Pou5f3.2 and Pou5f3.3; mPouV) or VegT (mVegT), and canonical Wnt, Nodal or BMP signal transduction, on zygotic transcription from MBT to late blastula and early gastrula stages (Fig. 6c and Supplementary Table 1). The high translation frequencies (Fig. 2a and Supplementary Fig. 2a) coupled with fairly steady or even transient (mVegT and Pou5f3.3) protein levels around MBT (Fig. 2d), suggest that maternal TFs and signal mediators have short half-lives. Consistent with this idea, the injection of antisense morpholino oligonucleotides (MOs) blocking the translation of maternal transcripts such as $S o x 3$ or $m V e g T$ was effective in reducing protein levels (Fig. 2c and Supplementary Fig. 2c,e,f). We used previously-validated MOs to knock down $\mathrm{mPouV}^{25}$ and $\beta$-catenin ${ }^{26}$. Although MOs can cause off-target mis-splicing and the induction of an immune response, the effects on splicing do not affect mature maternal transcripts and the immune response is only detectable beyond gastrula stages ${ }^{27}$. The nuclear accumulation of Smad1 and Smad2 in response to BMP and Nodal signaling was inhibited with the small molecules LDN193189 28,29 and SB431542 30,31 , respectively. The morphological defects of these loss-of-function (LOF) treatments ranged from undetectable (Sox3), to weak (mVegT, BMP), to moderate (mPouV), to severe (mPouV/Sox3, Nodal, $\beta$-catenin) (Fig. 6a and Supplementary Fig. 8a,c). Moderate and severe defects affected gastrulation, while weak ones were only obvious later. All phenotypes were either in line with previous publications $\left(\beta-\right.$ catenin $^{26}$, $\mathrm{Nodal}^{32}$ and $\mathrm{BMP}^{33}$ ) or could be rescued at the morphological (mPouV/Sox3; Fig. 6b) or transcriptional level (mVegT; Supplementary Fig. 8b) by co-injecting cognate mRNA. Interestingly, the role of maternal Sox3 could only be detected by knocking it down together with mPouV. In contrast to single Sox3 or mPouV LOF, double LOF embryos failed to close the blastopore (Fig. 6a and Supplementary Movie 1).

Transcriptome analysis of TF and signal LOF embryos was confined to the 3,687 zygotic genes for which $\geq$ two-fold reductions in exonic and/or intronic transcript level (see Online Methods) could be detected following injection of the RNAPII inhibitor $\alpha$ amanitin (Supplementary Table 9). About $83 \%$ of these genes had a maternal contribution of $\geq 1$ per 10 million transcripts as detected within first hour post-fertilization when the genome is quiescent (Fig. 6e). $\alpha$-Amanitin prevented any transcriptional changes and thus blocked the gastrulation movements that are normally initiated by ZGA (Fig. 6d and Supplementary Fig. 8d). The LOF-mediated reduction of ZGA ranged from $\sim 2 \%$ for BMP to $\sim 25 \%$ for mPouV/Sox 3 (Supplementary Fig. 11a). We note that the additional loss of Sox3 in mPouV LOF embryos further reduced ZGA of developmentally relevant genes, including those that are expressed ubiquitously, like mir427, and those that are activated in a subset of $\mathrm{mPouV} / \mathrm{Sox} 3^{+}$cells, like ventx and tbxt (Fig. 6f,g).

Spatial analysis of reduced gene activation confirmed that many of the $918 \mathrm{mPouV} / \mathrm{Sox} 3$-dependent genes showed enriched expression along the animal-vegetal or dorso-ventral axes (Fig. 7a,e). More specifically, comparison with signal LOFs revealed that 268 of the 708 genes induced by Wnt, Nodal or BMP also depended on mPouV/Sox3 (Fig. 7b and Supplementary Fig. 9 and 10b). Similarly, 175 of the $239 \mathrm{mVegT-dependent} \mathrm{genes,} \mathrm{including} \mathrm{such} \mathrm{as} \mathrm{foxal,} \mathrm{nodal5/6} \mathrm{and} \mathrm{sox} 17 a / b$, were activated in response to signaling (Supplementary Figs. 9 and 10a). Loss of mVegT and mPouV/Sox3 jointly affected 71 genes, 57 of which also depended on Wnt, Nodal or BMP signaling (Supplementary Fig. 10b).

Remarkably, the requirement for particular maternal TFs varied even among related genes with similar expression patterns that are activated by the same signals. For instance, tbxt and eomes are both activated by Nodal signaling in the marginal zone between the animal and vegetal hemispheres, but only tbxt requires mPouV/Sox3 for its signal responsiveness (Fig. 7a-c). Significantly, DNA occupancy levels did not explain the difference in gene regulation between $t b x t$ and eomes, because both gene loci showed high levels of Sox3 binding (see Sox3 track in Fig. 10a,b). Similar dependencies were found for Wnt-responsive genes like foxb1 and zicl (Supplementary Fig. 10c). We confirmed these mPouV/Sox3-dependent signal inductions by treating control and mPouV/Sox3-depleted animal cap tissue with Nodal (Activin) and Wnt (CHR99021) agonists (Fig. 7d and Supplementary Fig. 10d). Interestingly, mPouV/Sox 3 also facilitated basal low-level expression of (especially) tbxt and foxbl without Nodal or Wnt stimulation.

Together, our selected maternal TFs and signals activated 1,473 of the 3,687 ( 40\%) zygotic genes. These included substantial percentages of genes with preferential expression in the animal $(\sim 62 \%)$, the vegetal $(\sim 89 \%)$, the dorsal $(\sim 95 \%)$ and the ventral 

tend to affect biological functions for which signal-induced regionalization of ZGA is essential, such as the formation of the main body axes and the segregation of the three germ layers (Supplementary Fig. 11b). We also note that LOF of mPouV/Sox 3 caused significant increases in transcript levels of genes encoding gamete-specific biological processes, suggesting that reprogramming towards embryonic pluripotency was compromised (Supplementary Fig. 11a,b).

\section{Pioneering activity of pluripotency TFs predefines signal-mediated gene induction}

To discover how maternal TFs allow cell type-specific genes to be signal-induced, we compared various chromatin features from genome-wide accessibility (DNase-Seq; Fig. 8a and Supplementary Fig. 12a) and DNA occupancy (ChIP-Seq for H3K4me1, RNAPII, Smad2 and $\beta$-catenin; Fig. 8a) to the high-resolution conformation contacts (next-generation capture-C; Fig. 9a, LOF embryos at MBT (Supplementary Table 1). We selected mPouV/Sox3 rather than $\mathrm{mVegT}$ because of their stronger effect on the ZGA (Supplementary Fig. 11a). Genome-wide analysis showed that mPouV/Sox3 LOF strongly reduced chromatin accessibility as measured by the significant (FDR $\leq 10 \%$ ) loss of DNase cleavages in 6,738 of the 16,637 ( 41\%) pCRMs (Fig. 8b,c and Supplementary Table 11). Sorting of these pCRMs according to the significance of lost accessibility (Fig. 8d) suggests that chromatin opening depends on the pioneering activity of mPouV and Sox 3 to recognize their canonical motifs in compacted chromatin (Fig. 8e). By comparison, unaffected pCRMs contained canonical POU/SOX motifs less frequently, and were strongly enriched for promoter-centric motifs of the Krüppel ZF, bZIP and NFY protein families (Fig. 8e,f). At the extreme end of affected loci (e.g. $t b x t$, foxb1, $c d c 25 b$ and $z i c 1$ ) entire or large proportions of super-enhancers became inaccessible upon mPouV/Sox $3 \mathrm{LOF}$ (Figs. 9d and 10a and Supplementary Figs. 13 and 14).

The loss of accessibility triggered further profound changes to chromatin in situ (see arrowheads at bottom of Figs. 9d and 10a and Supplementary Figs. 13 and $\mathbf{1 4}$ for strongly affected pCRMs). First, the deposition of H3K4me1, RNAPII and signal mediators Smad 2 and $\beta$-catenin was substantially reduced (Fig. 8d and 9d and Supplementary Fig. 13 and 14). Second, in contrast to unaffected pCRMs, 32 pCRMs with compromised accessibility—most of which are part of super-enhancers-also showed significantly (FDR $\leq 10 \%$ ) reduced promoter contacts (e.g., tbxt, foxbl, $c d c 25 b$ and $z i c 1$ ) (Figs. 9c,d and 10a, Supplementary Figs. 13 and 14 and Supplementary Table 12). Third, at the transcriptional level, lower usage of (clustered) pCRMs coincided with the reduction of coding (Fig. 8g) as well as local non-coding RNA (last column in Fig. 8d and 'low RNA' track in Fig. 9d and Supplementary Figs. 13 and 14). Importantly, the profiling of LOF embryos revealed how chromatin predetermines signal induction and why, for instance, Nodal-induced transcription of $t b x t$ was strongly affected by the loss of mPouV/Sox 3 and that of eomes less so. In contrast to those of eomes, all promoter-tied pCRMs (super-enhancer) of $t b x t$ contain canonical POU/SOX motifs, so were not accessible to Smad2 in mPouV/Sox3 LOF embryos (Fig. 10a,b). Thus, Smad2 interactions with critical Nodal responsive CRMs of eomes remained intact, but those of $t b x t$ were impeded by compacted chromatin. Pioneer-initiated competence also applied to Wnt signaling with $\beta$-catenin failing to engage with Wnt responsive CRMs of foxb1 in the absence of mPouV/Sox3 (Supplementary Fig. 13). Other transcriptional deficiencies in signal-responsive or non-responsive genes that were triggered by mPouV/Sox3 LOF also coincided with significant reductions (FDR $\leq 10 \%$ ) in chromatin accessibility (Fig. 10c and Supplementary Figs. 15, 16 and 17), suggesting that mPouV/Sox3 facilitates expression by making critical clusters of CRMs accessible to signal and other transcriptional mediators.

\section{DISCUSSION}

Our results allow us to propose a model (Fig. 10d) of pioneer-initiated chromatin remodeling, or priming, that unlocks contextspecific CRMs, some of which contain signal-responsive elements that enhance and regionalize transcription. Our observations are in line with recent reports of CRM priming for tissue-specific gene expression ${ }^{34}$ and signal interpretation ${ }^{35,36}$. Thus, cell typespecific TFs pre-determine signal interpretation by shifting the binding of signal mediators and so causing the induction of different genes. These, ultimately, result in different cell fate transitions. Developmental context is imprinted on chromatin by co-expressed 
partial DNA motifs exposed on nucleosome surfaces can be enough to initiate chromatin access. Subsequent displacement of nucleosomes may be driven by cooperation among several TFs and the higher affinity of TFs for free genomic DNA. In the context of establishing pluripotency before MBT, we show that the pioneering activity of maternal Pou5f3 (mPouV) and Sox3 recognizing their POU/SOX motifs (Fig. 10d, left branch in top panel) triggers extensive chromatin remodeling in situ. This includes the H3K4me1 marking of CRM-flanking nucleosomes and chromatin looping with promoters. Newly primed CRMs can initiate further canonical as well as opportunistic (sequence-nonspecific) binding. Thus, irrespective of their preference for certain DNA sequences, chromatin factors (e.g. TFs, signal mediators, RNAPII) can appear on accessible CRMs with no canonical DNA binding motifs (Fig. 10d, right branch in top panel). However, the opportunistic recruitment of mPouV and Sox3, at least, seems to have little to no effect on changing the chromatin landscape and its transcriptional readout. This may be also true for other TFs as shown for ectopic MyoD in our study. Such recruitment behaviors can be difficult to detect without multi-level analysis of chromatin under LOF conditions. Overall, the canonical binding of mPouV/Sox3 strongly contributes to the pioneering of two fifths of all accessible pCRMs at MBT which permits or instructs one quarter of the ZGA (Fig. 8b,g). We often observe that the usage of pCRMs also coincides with the low-level activation of proximal non-coding RNA.

Although we have not performed chromatin profiling at a single-cell level, we suggest for three reasons that this priming occurs in every embryonic cell before the onset of regional Wnt, Nodal or BMP signaling. First, the nuclear accumulation of maternal TFs (e.g., mPouV, Sox3, Foxh1 and mVegT) occurs ubiquitously (or within the vegetal hemisphere in case of mVegT) and precedes that of signal mediators. Second, mPouV/Sox3 LOF affects chromatin similarly at zygotic genes with (e.g. foxbl) or without (e.g. $c d c 25 b$ ) tissue-specific expression. And finally, dissected animal caps replicate the in vivo response of the marginal zone tissue to Nodal and Wnt signaling. Importantly, and consistent with the idea of context-dependent signal interpretation, our findings suggest that signal mediators have inferior pioneering activity and thus rely on cooperating pioneer factors like $\mathrm{mPouV}$ and Sox 3 to make their signal response elements accessible. Analysis of the related genes eomes and tbxt, both of which are induced by Nodal signaling, but with only tbxt depending on $\mathrm{mPouV} / \mathrm{Sox} 3$, reveals that the same context-dependent competence can be differentially encoded in the genome (through the presence or absence of POU/SOX motifs in signal-responsive CRMs) (Fig. 10d, lower panel). Based on transcriptional dependencies shown here and in the spinal cord ${ }^{38}$, this regulation of competence is complemented by other TFs like mVegT and also applies to Wnt signaling and other signaling pathways such as BMP and Sonic hedgehog.

Our work has focused on the pioneering activity of mPouV and Sox3. The enrichment of DNA recognition motifs at pCRMs in use before the MBT suggests that only a few additional maternal TF families, including those of T-box, FoxH and Krüppel-like ZF families, are involved in the bulk acquisition of competence at the MBT (Fig. 2b). Indeed, many of these TF family members are rapidly turned over and are most abundant around the $\mathrm{MBT}^{39,40}$, suggesting dynamic and strong TF activity ${ }^{41}$. Their activity generates a signature of accessible DNA motifs of pluripotency or early cell lineage determination that is conserved among vertebrates and is reminiscent of the chromatin footprints in embryonic stem cells in vitro ${ }^{42}$. Remarkably, as in the human genome $^{42}$, POU/SOX motifs populate distal CRMs, while Krüppel ZF motifs are frequently found in promoter-proximal CRMs

(Fig. 8f). This creates a functional separation among the pluripotency factors where PouV/SoxB1 and Krüppel-like factors remodel enhancers and promoters, respectively. Collectively, we show that $\mathrm{mPouV} / \mathrm{Sox} 3$ predetermine cell fate decisions by initiating access to signal-responsive CRMs via POU/SOX motifs. The use of these permissive CRMs is most prominent in pluripotent cells $\mathrm{s}^{24,43}$ and proves to be critical in vivo to the induction of zygotic genes with functions in germ layer formation and primary axis determination.

The findings presented here confirm that core pluripotency TFs contribute substantially to ZGA as previously reported for SoxB1, PouV and Nanog TFs for zebrafish ZGA ${ }^{44,45}$. However, in contrast to zebrafish or humans, a true nanog gene has not been found in the Xenopus genome, which has led to the suggestion that zygotic Ventx TFs with their Nanog-like DNA binding characteristics could operate as the gatekeepers of pluripotency instead ${ }^{46}$. This notwithstanding, we anticipate that signal competence is controlled across vertebrate embryonic stem cells in a manner resembling that in the early Xenopus embryo.

The approach we adopt here can be applied to any other cell type to discover the molecular basis of its competence. Ultimately, this will generate a lexicon of competence that outlines which pioneer factors unlock which (signal-responsive) CRMs and gene 
303 loci. For example, the motif compositions of CRMs later engaged in the Xenopus tailbud embryo points at the potential of OTX

304 and CDX TFs in conveying competence in anterior and posterior compartments, respectively (Fig. 5c). Interestingly, various 305 tumors are associated with the mis-expression of pioneer factors ${ }^{47}$ and thus may display different competencies from the

306 surrounding host tissue. Conversely, knowing which pioneers are required to permit a specific signal response will increase the success of engineering patient-specific tissue for transplantation therapy. On a broader level, our profiling of chromatin states under LOF conditions discriminates functional from non-functional binding and provides a promising avenue for deciphering the non-coding part of the genome for basic and therapeutic research.

\section{METHODS}

312 Methods and any associated references are available in the online version of the paper.

313 Accession code. Sequencing data are deposited in the Gene Expression Omnibus (GEO) database with accession number 314 GSE113186

\section{ACKNOWLEDGMENTS}

317 We thank Abdul Sesay, Leena Bhaw, Harsha Jani, Deborah Jackson and Meena Anissi for deep sequencing; Mike Klymkowsky,

318 Manolis Gialitakis and John Gurdon for supplying antibodies; Stefan Hoppler and Yukio Nakamara for discussing $\beta$-catenin ChIP

319 protocol; Jim Hughes and Damien Downes for sharing pre-publication results of an improved next-generation capture-C method;

320 Ying Wang for providing sequence conservation tracks; Mareike Thompson, Rita Monteiro and Clara Collart for critical reading of the manuscript; and the Smith lab for discussions and advice. G.E.G and J.C.S. were supported by the Medical Research Council (program number U117597140) and are now supported by the Francis Crick Institute, which receives its core funding from Cancer Research UK (FC001-157), the UK Medical Research Council (FC001-157), and the Wellcome Trust (FC001-157).

\section{AUTHOR CONTRIBUTIONS}

326 Conceptualization, G.E.G.; Methodology, G.E.G.; Software, G.E.G.; Formal Analysis, G.E.G. and N.D.L.O.; Investigation,

\section{COMPETING FINANCIAL INTERESTS}

331 The authors declare no competing financial interests. 
a

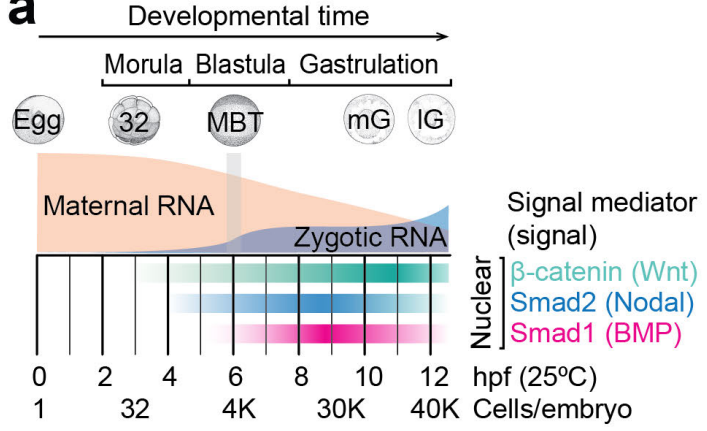

\section{d}

Correlation of RNAPII+ pCRMs

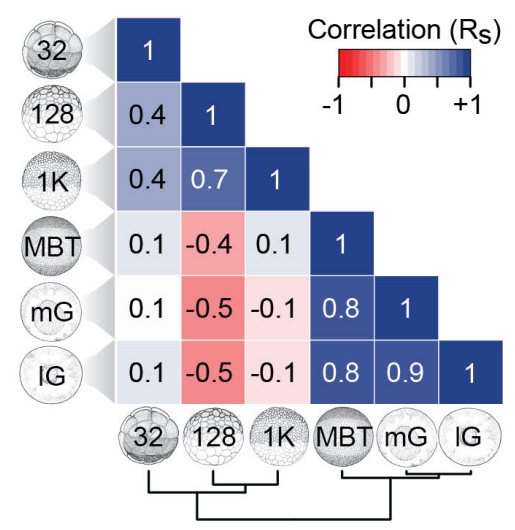

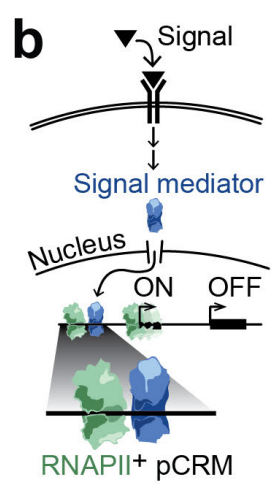

e

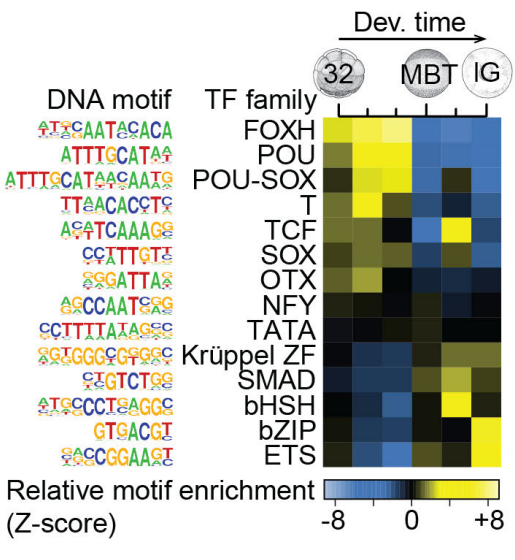

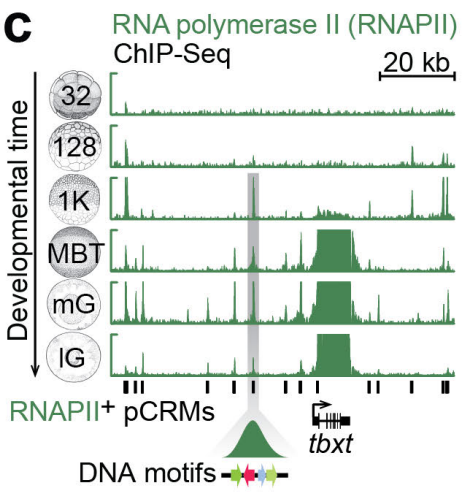

f

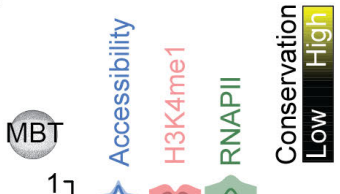

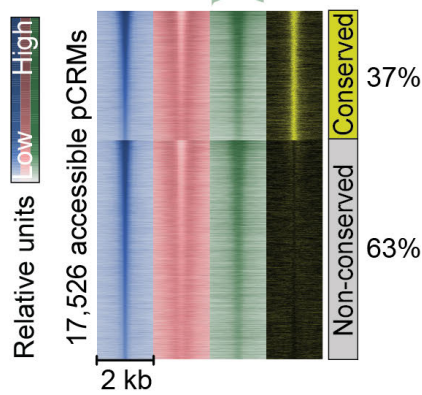

Figure 1 Characterization of pCRMs instructing ZGA. (a) Timeline (hpf, hours post-fertilization, at $25^{\circ} \mathrm{C}$ ) of the maternalto-zygotic transition and earliest signaling events (nuclear accumulation of Wnt, Nodal and Bmp signal mediators $\beta$-catenin, Smad2 and Smad1, respectively) during early X. tropicalis development up to the late gastrula embryo (12 hpf) with $\sim 40,000$ (40K) cells. (b) Signal transduction pathway causing signal mediators to enter the nucleus and engage with pCRMs (e.g. marked by RNAPII). (c) Snapshot of RNAPII recruitment to pCRMs of the zygotic gene locus tbxt from the 32-cell to the late gastrula stage. The underlying DNA sequence of RNAPII ${ }^{+}$pCRMs are used to discover enriched DNA motifs de novo (illustrated as colored arrows for one RNAPII ${ }^{+}$pCRM). (d) Spearman correlations $\left(\mathrm{R}_{\mathrm{s}}\right)$ of RNAPII binding levels across $\sim 27,000$ pCRMs (Supplementary Table 2) between the indicated developmental stages. (e) Temporal enrichment (Z-score) of consensus DNA motifs known to be recognized by indicated TF families among RNAPII ${ }^{+}$pCRMs. (f) MBT-staged heat map of DNase-probed chromatin accessibility ( $\mathrm{n}=2)$, RNAPII binding and H3K4me1 ( $\mathrm{n}=2)$ marking across $\sim 17,500 \mathrm{pCRMs}$ (Supplementary Table 3) grouped by sequence conservation levels (phastCons) and sorted by the statistical significance of pCRM accessibility. Abbreviations used for the developmental timeline: 32, 128 and 1K, 32-, 128- and 1,024-cell stage; MBT, mid-blastula transition; eG, $\mathrm{mG}$ and $\mathrm{lG}$, early, mid- and late gastrula stage. 
bioRxiv preprint doi: https://doi.org/10.1101/306803; this version posted June 3, 2019. The copyright holder for this preprint (which was not certified by peer review) is the author/funder, who has granted bioRxiv a license to display the preprint in perpetuity. It is made available under aCC-BY 4.0 International license.

a

Sequence-specific TFs \& signal mediators Other RNAPII-regulating factors

Remaining proteins

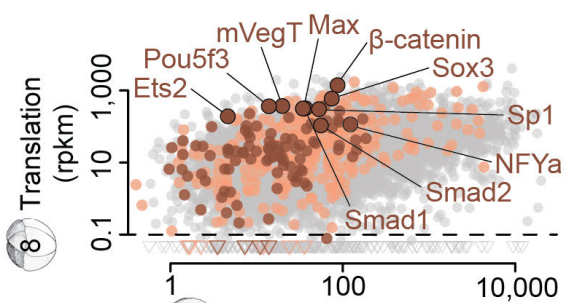

Egg Protein concentration (nM)

\section{b}

\begin{tabular}{|c|c|}
\hline $\begin{array}{c}\text { Most enriched DNA } \\
\text { motifs at pCRMs }\end{array}$ & \begin{tabular}{|l} 
Highest rates of protein \\
synthesis before MBT
\end{tabular} \\
\hline TF family & Member \\
\hline $\begin{array}{ll} & \text { POU-SOX } \\
- & \text { Krüppel ZF } \\
: & \text { POU } \\
: \text { SOX } \\
: \text { bZIP } \\
: \text { FOXH } \\
: \text { ETS } \\
: \text { NFY } \\
: \text { SMAD } \\
: \text { TCF } \\
\therefore \text { THSH } \\
: \text { OTX }\end{array}$ & $\begin{array}{l}\text { Pou5f3-Sox3 } \\
\text { Sp1, several KIf } \\
\text { Pou5f3 } \\
\text { Sox3 } \\
\text { Max } \\
\text { Foxh1 } \\
\text { Ets2 } \\
\text { NFYa/b/c } \\
\text { Smad1/2 } \\
\text { mVegT } \\
\text { Tcf/ } \beta \text {-catenin } \\
\text { Tfap2 } \\
\text { Otx1 } \\
\end{array}$ \\
\hline $\begin{array}{l}\% \text { coverage: } \\
510 \\
-10\end{array}$ & $\begin{array}{l}\text { ved pCRMs } \\
\text { nserved pCRMs } \\
\text { ound regions }\end{array}$ \\
\hline
\end{tabular}

C

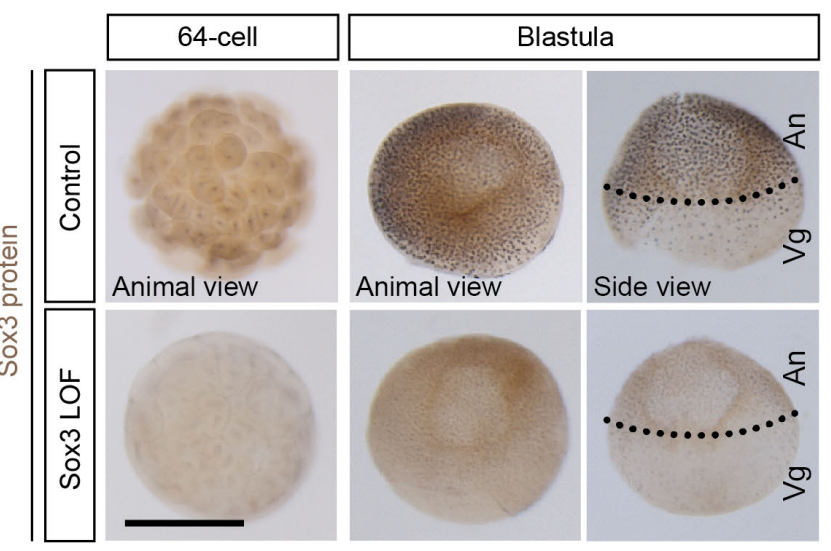

d

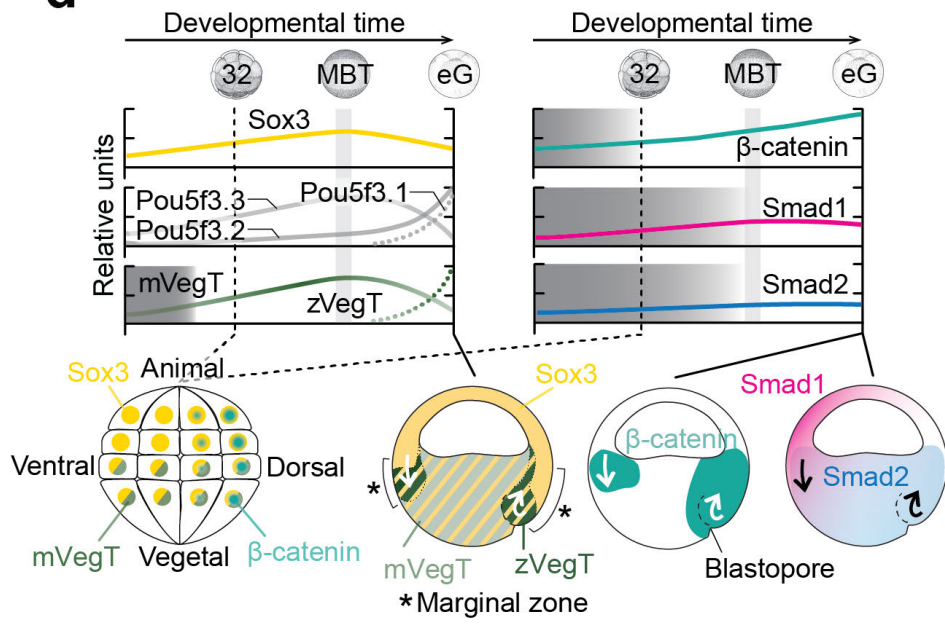

Figure 2 Search for ZGA-critical proteins based on their significantly enriched DNA recognition motifs at accessible and engaged (RNAPII ${ }^{+} / \mathrm{H} 3 \mathrm{~K} 4 \mathrm{me}^{+}$) pCRMs and their high translation frequency before MBT. (a) Maternal protein concentrations in the egg $^{12}$ versus ribosome footprint (translation) levels at the 8 -cell stage ${ }^{13}$. Most frequently translated representatives of various TF families are labeled. (b) Matching canonical pCRM-enriched DNA motifs (sorted by statistical significance) with frequently translated TFs and signal mediators. (c) WMIHC of Sox 3 protein in control and Sox3 loss-offunction (LOF) embryos at the 64-cell and blastula stage. Nuclear accumulation of Sox3 protein was detected in both the animal (An) and vegetal (Vg) hemisphere of control embryos. Scale bar, $0.5 \mathrm{~mm}$. (d) Graphical illustration of protein levels (derived from mass spectrometry data ${ }^{40}$ ) and nuclear localizations (mainly derived from WMIHC, see references below) of selected TFs and signal mediators based on our and previously published results: Sox3 (this study and ref. ${ }^{48}$ ), mPouV (Pou5f3.2 and Pou5f3.3) and (zygotic) Pou5f3.1 (deduced from transcript data ${ }^{14}$ ), mVegT and zVegT ${ }^{15}, \beta$-catenin ${ }^{9,16}$, Smad1 $^{1}$ (this study and ref. ${ }^{9,17}$ ) and Smad2 (this study and ref. ${ }^{9,17}$ ). Shaded boxes indicate periods of non-nuclear protein localization. Arrows indicate tissue movements of gastrulation. Abbreviations used for the developmental timeline: 8 and 32 , 8-cell and 32-cell stage; MBT, mid-blastula transition; and eG, early gastrula stage. 
bioRxiv preprint doi: https://doi.org/10.1101/306803; this version posted June 3, 2019. The copyright holder for this preprint (which was not certified by peer review) is the author/funder, who has granted bioRxiv a license to display the preprint in perpetuity. It is made available under aCC-BY 4.0 International license.

a

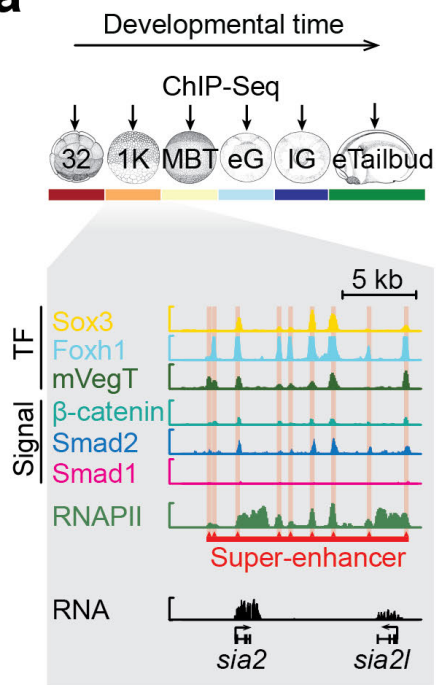

b

Biological functions of super-enhancer ${ }^{+}$genes

\begin{tabular}{ll|l|l|} 
Dev. time & \multicolumn{2}{l}{ Number of zygotic genes } \\
\hline $5-10|11-50| 51-100$ & $101-500$ \\
Embryonic morphogenesis \\
Cell fate commitment \\
Pattern specification process \\
Anterior/posterior pattern specification \\
Dorsal/ventral pattern formation \\
Gastrulation \\
Stem cell differentiation \\
Animal organ morphogenesis \\
Formation of primary germ layer \\
Enithelium development \\
Mesoderm development \\
Nodal signaling pathway \\
Response to BMP \\
$10-60$ Significance (p-value)
\end{tabular}

C

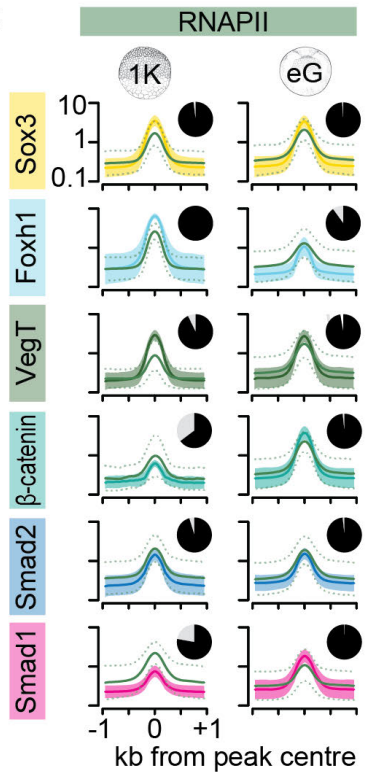

d

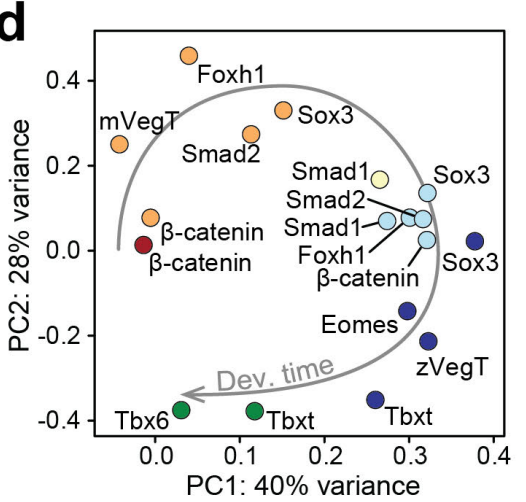

e

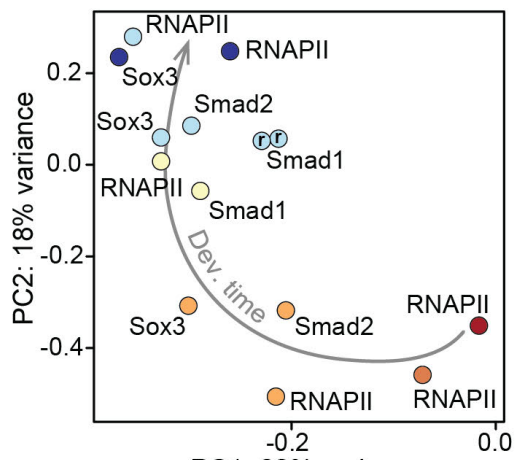

PC1: $60 \%$ variance f

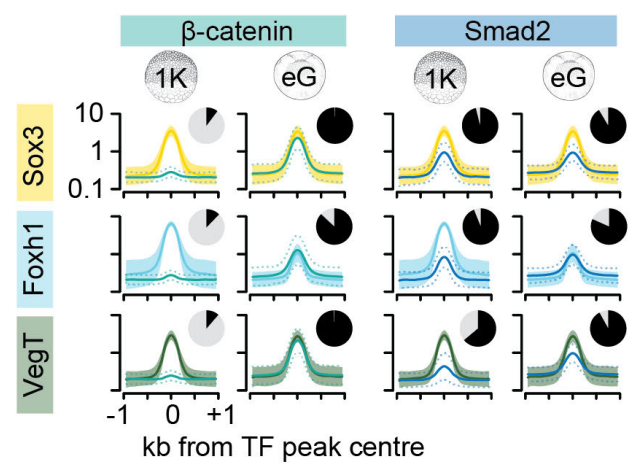

g

$\mathrm{kb}$ from TF peak centre

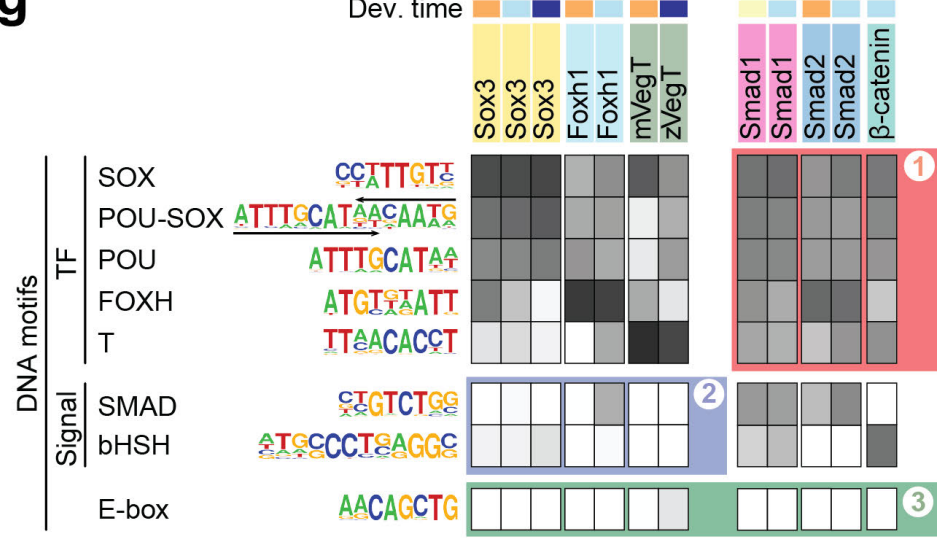

Dev. time $\frac{\text { TF }}{\square-\square}$
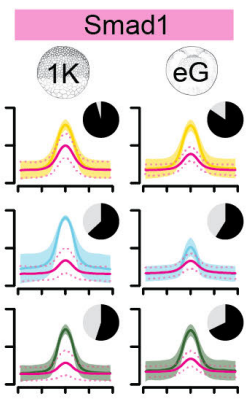

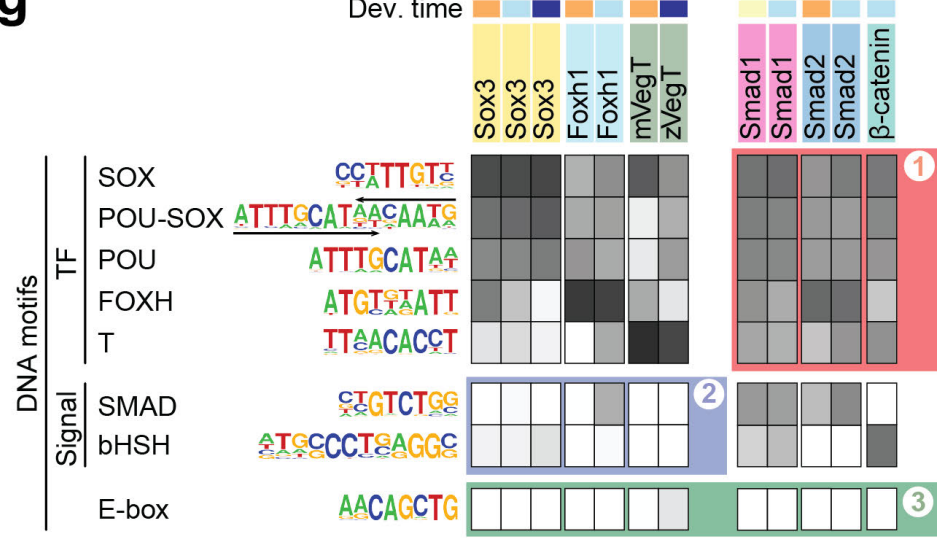

Significance of motif enrichment Low_High

Figure 3 Chromatin engagement of TFs and signal mediators during the maternal-to-zygotic transition. (a) Chromatin profiling (ChIP-Seq, $n=1-3$ ) of selected TFs and signal mediators from the 32-cell to the early tailbud stage. In all subsequent figure panels, the chromatin factors and developmental stages profiled are consistently color-coded as illustrated here. The excerpt of multiple chromatin tracks shows the binding of maternal TFs (Sox3, Foxh1 and VegT) and signal mediators ( $\beta$ catenin, Smad2 and Smad1) to the siamois2 (sia2 and sia2l) super-enhancer at the 1,024-cell stage (see Supplementary Fig. 3a for the temporal progression of chromatin engagement to the siamois 2 and ventx super-enhancers). (b) Bubble plot shows significantly enriched biological processes associated with zygotic super-enhancer ${ }^{+}$genes (i.e. genes possessing engaged super-enhancers $\leq 5 \mathrm{~kb}$ from their active TSS at indicated developmental stages). (c) Meta-plots (mean [solid line] $\pm \mathrm{SD}$ [dotted 
bioRxiv preprint doi: https://doi.org/10.1101/306803; this version posted June 3, 2019. The copyright holder for this preprint (which was not certified by peer review) is the author/funder, who has granted bioRxiv a license to display the preprint in perpetuity. It is made available under aCC-BY 4.0 International license.

371 line or polygon]) summarize the level of RNAPII engagement across 2,000 pCRMs most frequently occupied by the indicated 372 TFs or signal mediators at the 1,024-cell and early gastrula stage, respectively. The pie chart next to each meta-plot shows the 373 percentage of these $\mathrm{TF}^{+}$or signal mediator ${ }^{+}$pCRMs bound by RNAPII (ChIP $\geq 2 \mathrm{x}$ input tag density): 98\% (1,024-cell stage) 374 and $99 \%$ (early gastrula stage) of Sox $3^{+}$pCRMs, $100 \%$ and $90 \%$ of Foxh $1^{+}$pCRMs, $93 \%$ and $97 \%$ of $\mathrm{VegT}^{+} \mathrm{pCRMs} 65 \%$ 375 and $98 \%$ of $\beta$-catenin ${ }^{+}$pCRMs, $96 \%$ and $98 \%$ of Smad $2^{+}$pCRMs, and $78 \%$ and $99 \%$ of Smad $1^{+}$pCRMs. (d) Biplot of principal 376 component (PC) 1 (accounting for $40 \%$ variance) and 2 (28\% variance) shows the similarity (or dissimilarity) of TF (Sox3, $377 \mathrm{mVegT}$, Foxh1 ${ }^{18,25}$, Eomes ${ }^{19}, \mathrm{zVegT}^{19}, \mathrm{Tbxt}^{19}$ and Tbx6) and signal mediator ( $\beta$-catenin, Smad1, Smad2) binding levels across $378 \sim 12,500$ highly engaged pCRMs (compiled from the 2,000 pCRMs with the highest DNA occupancy levels detected per 379 protein and developmental stage) over several developmental stages. Note that developmental time (arrow) separates these 380 profiles best. (e) Biplot of PC1 (accounting for 60\% variance) and PC2 (18\% variance) for the temporal progression of RNAPII 381 and TF binding levels across the same set of pCRMs as in (d). Abbreviation: r, biological replicates. (f) Meta-plots (mean 382 [solid line] \pm SD [dotted line or polygon]) summarizes the level of signal mediator engagement across 2,000 pCRMs most 383 frequently occupied by the indicated $\mathrm{TF}$ at the 1,024-cell and early gastrula stage, respectively. The pie chart next to each 384 meta-plot shows the percentage of these $\mathrm{TF}^{+}$pCRMs bound by signal mediators (ChIP $\geq 2 \mathrm{x}$ input tag density): $\beta$-catenin at 385 Sox $3^{+}$pCRMs ( $10 \%$ at the 1,024 -cell stage and $100 \%$ at the early gastrula stage), Smad 2 at Sox $3^{+}$pCRMs (96\% and $\left.91 \%\right)$, 386 Smad1 at Sox $3^{+}$pCRMs (96\% and 85\%), $\beta$-catenin at Foxh $1^{+}$pCRMs (12\% and 87\%), Smad 2 at Foxh $1^{+}$pCRMs $(94 \%$ and

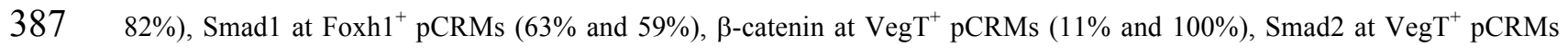
388 (64\% and 92\%) and Smad1 at $\mathrm{VegT}^{+} \mathrm{pCRMs}$ (55\% and 68\%). (g) Heat map shows the statistical significance of finding TF389 and signal-specific DNA consensus motifs (y-axis) across 2,000 pCRMs most frequently occupied by the indicated TFs or 390 signal mediators (x-axis). Abbreviations used for the developmental timeline: 32 and 1K, 32-cell and 1,024-cell stage; MBT, 391 mid-blastula transition; eG and $1 \mathrm{G}$, early and late gastrula stage; and eTailbud, early tailbud. 
a

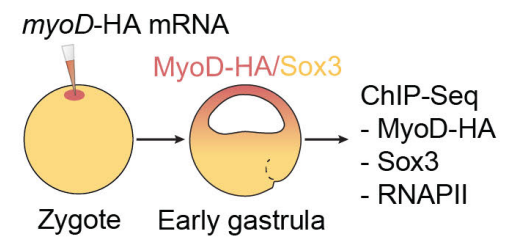

b

$\Delta$ Sox3 RNAPII $\square$ ectopic MyoD

$\rightarrow$ Endogenous dynamics

$\longrightarrow$ Enforced changes by MyoD

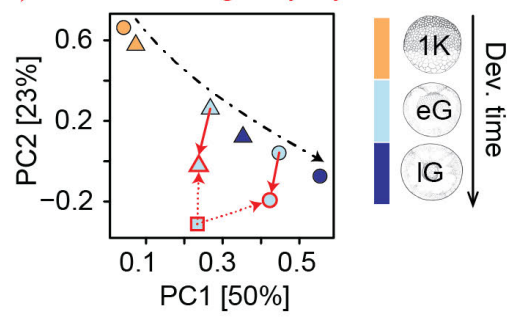

d

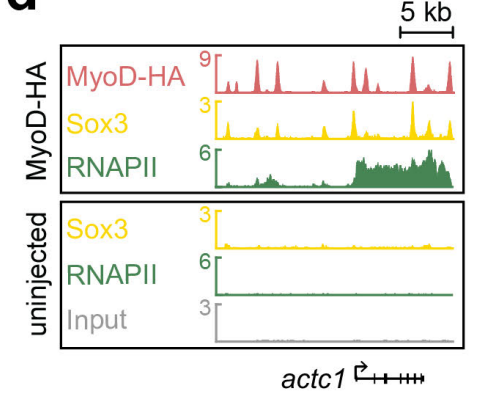

g

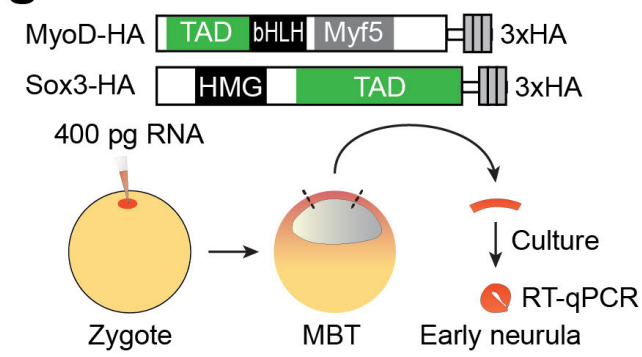

C

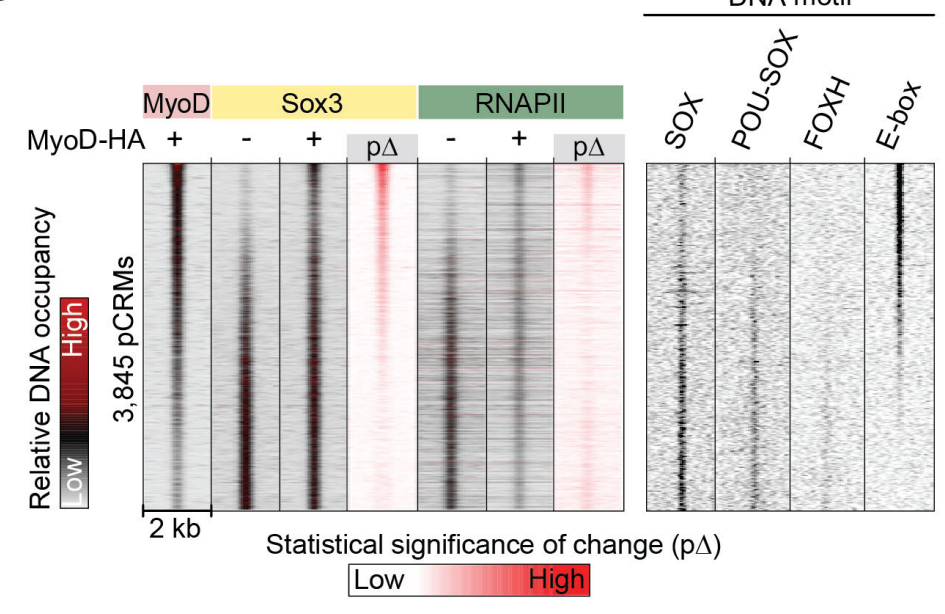

e

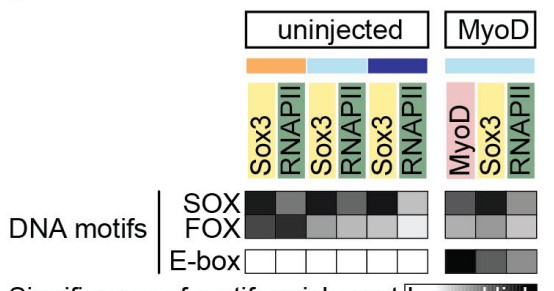

Significance of motif enrichment Low High f

h

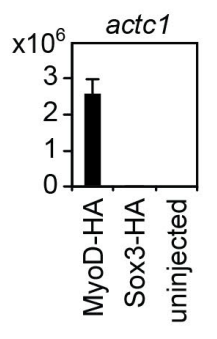

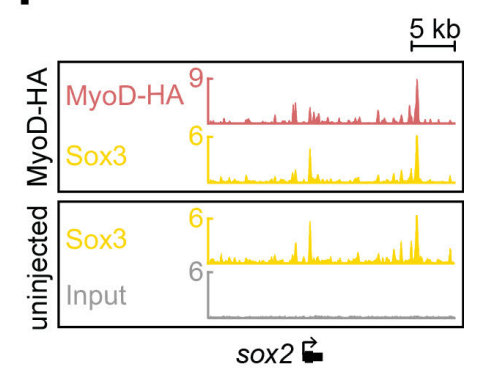

Figure 4 Ectopic expression of the muscle determinant MyoD reveals the effect of co-expressed TFs on chromatin engagement and gene expression. (a) Experimental design: Ectopic expression of the MyoD-HA mRNA construct injected into the animal hemisphere followed by the genome-wide chromatin profiling (ChIP-Seq; n=2) of MyoD-HA, Sox3 and RNAPII in early gastrula embryos. (b) Biplot of principal component (PC) 1 (accounting for 50\% variance) and 2 (23\% variance) shows the relationship of Sox3 (triangle), RNAPII (circle) and MyoD-HA (square) binding levels across MyoD ${ }^{+}$ and/or Sox $3^{+}$pCRMs. Arrows show the normal temporal dynamics of Sox3 and RNAPII binding (black dash-dotted line) and the MyoD-enforced (red dotted line) changes to them (red solid line) at early gastrula stage. Fill color of symbols represents the developmental stage as indicated, while line colors refer to whether MyoD-HA was expressed (red) or not (black). Abbreviations used for the developmental timeline: $1 \mathrm{~K}, 1,024$-cell stage; eG and $1 \mathrm{G}$, early and late gastrula stage. (c) Heat map of the DNA occupancies of 3,845 pCRMs and enriched DNA motifs sorted by the significance of MyoD-HA-enforced changes $(\mathrm{p} \Delta)$ to Sox 3 binding levels. (d,f) Snapshot of chromatin co-recruitment to the super-enhancers of canonical MyoD and Sox 3 target genes actc1 and sox2, respectively. (e) Heat map shows the significance of DNA motif enrichments (y-axis) at 10,000 pCRMs most frequently occupied by the indicated proteins in uninjected and MyoD-HA injected embryos (x-axis). 
bioRxiv preprint doi: https://doi.org/10.1101/306803; this version posted June 3, 2019. The copyright holder for this preprint (which was not certified by peer review) is the author/funder, who has granted bioRxiv a license to display the preprint in perpetuity. It is made available under aCC-BY 4.0 International license. animal cap assay. Error bars, mean+SD $(\mathrm{n}=2)$.

a

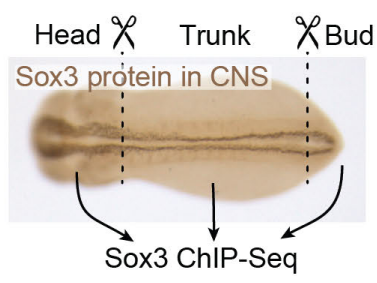

b

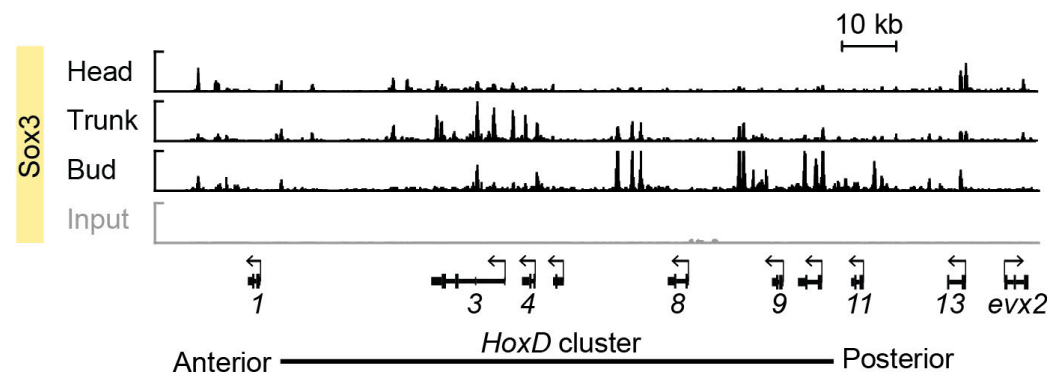

C

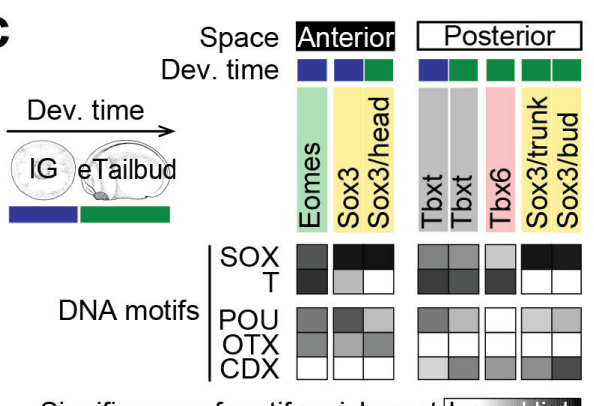

d

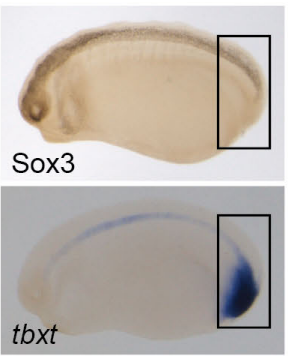

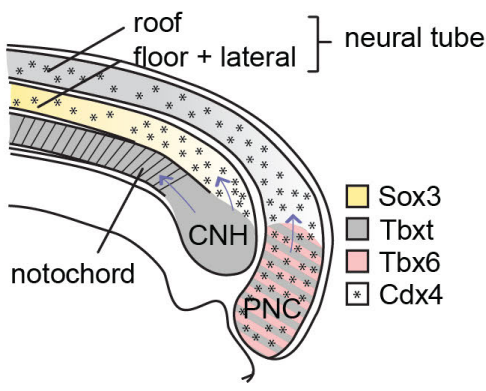

Figure 5 Profiling chromatin for Sox 3 in different anterior-posterior compartments of the central nervous system (CNS). (a)

410 Experimental design: Genome-wide Sox3 profiling of head, trunk and bud dissected from early tailbud embryos after tissue

411 fixation. (b) Snapshot of Sox3 binding to HoxD cluster in the head, trunk and bud. Note the differential binding of Sox3 with

412 'anterior', 'middle' and 'posterior' Hox genes being preferentially occupied in head, trunk and bud, respectively. (c) Heat map

413 shows the significance of DNA motif enrichments (y-axis) across 10,000 pCRMs most frequently occupied in each chromatin

414 profile (x-axis). Profiles are grouped depending on whether the cells expressing the TF of interest preferentially contribute to 415 the anterior or posterior compartment. Abbreviations used for the developmental timeline: 1G, late gastrula; and eTailbud, 416 early tailbud. (d) Anatomical map of TF expression at the posterior end of an early tailbud embryo to explain the co-enrichment 417 of 'posterior' TF-specific DNA recognition motifs in (c). Sox3-expressing cells of the posterior neural tube originate from the 418 chordoneural hinge $(\mathrm{CNH})$ and posterior wall of the neurenteric canal (PNC) expressing Brachyury and Brachyury/Tbx6, respectively ${ }^{20}$. Both neural tube and PNC are also exposed to the expression of $\mathrm{Cdx}$ such as $\mathrm{Cdx} 4^{49}$. 
bioRxiv preprint doi: https://doi.org/10.1101/306803; this version posted June 3, 2019. The copyright holder for this preprint (which was

not certified by peer review) is the author/funder, who has granted bioRxiv a license to display the preprint in perpetuity. It is made available under aCC-BY 4.0 International license.

a
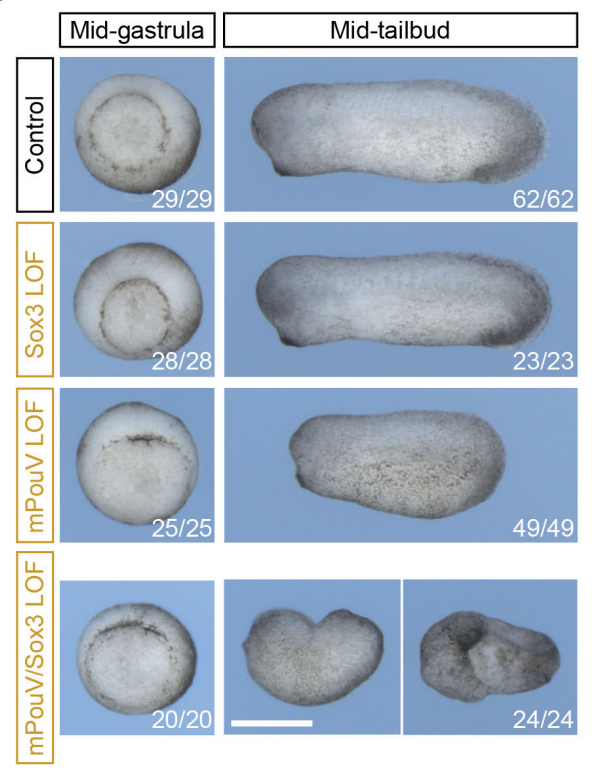

b
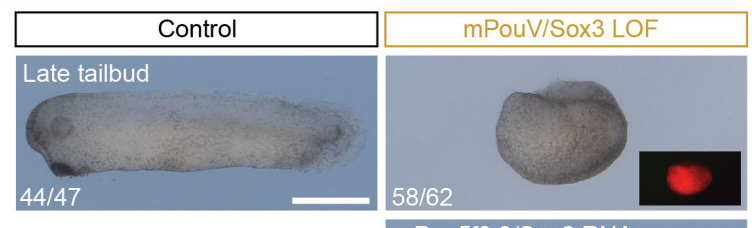

C

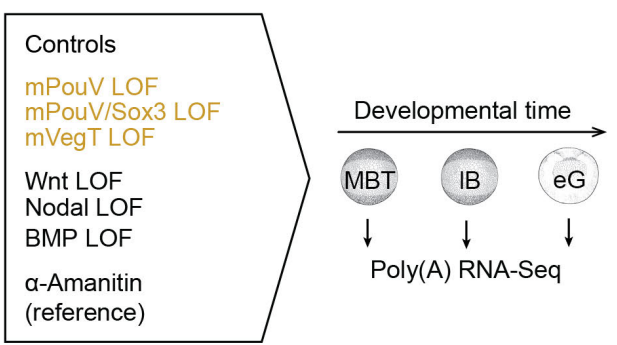

d
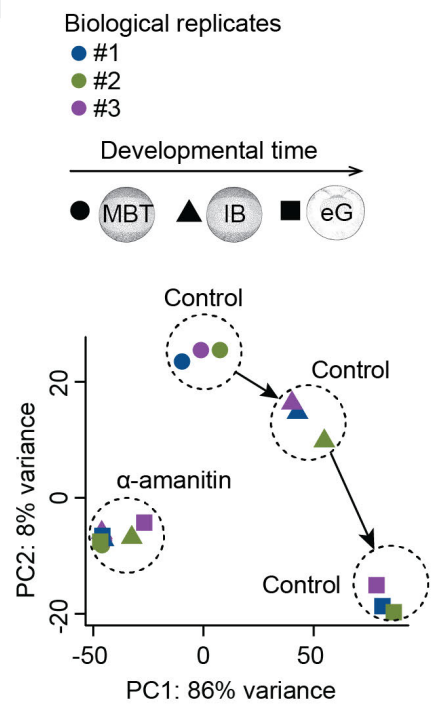

f

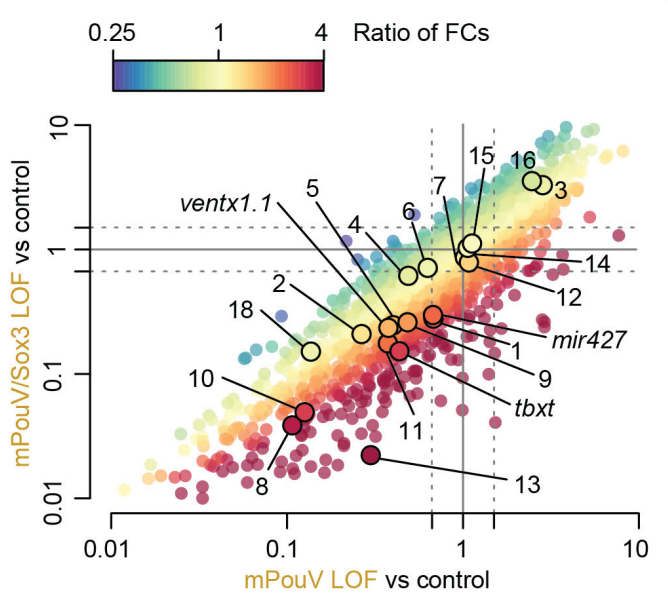

e

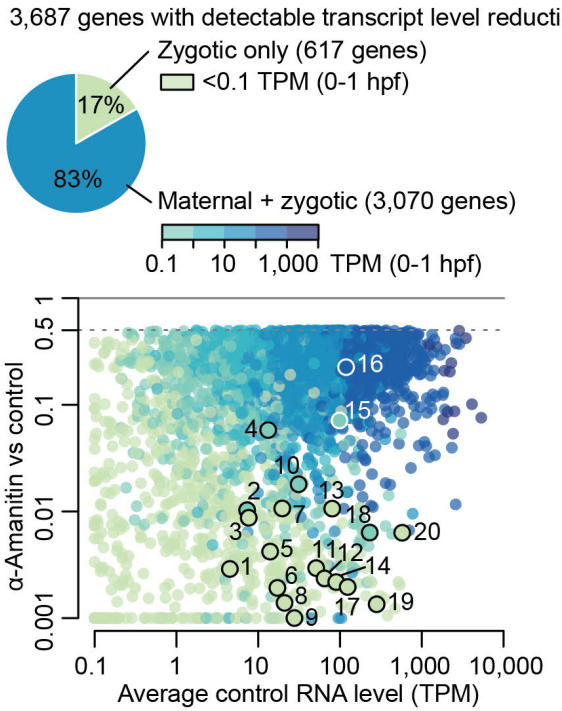

(1) foxh1.2

(2) fgf20

(3) sia2

(4) $h n f 1 b$

(5) foxd3

(6) nodal3

(7) gata2

(8) zeb2

(9) foxa1

(10) szl

(11) foxi4. 2

(12) eomes

(13) $c d x 4$

(14) chrd

(15) otx2

(16) id2

(17) ventx1.1

(18) sox2

(19) tbxt

(20) mir427

g

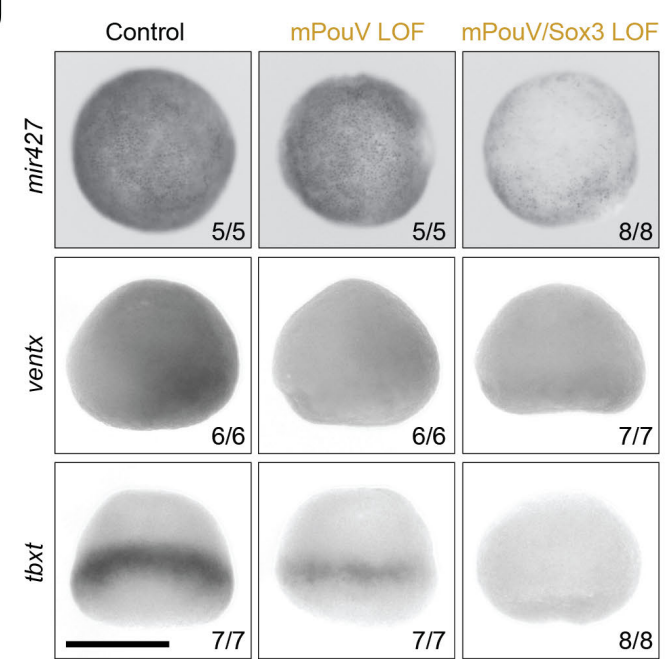

Figure 6 Synergistic relationship between maternal Pou5f3 (mPouV) and Sox3. (a) Morphological phenotypes caused by

single and combined LOFs of Sox 3 and $\mathrm{mPouV}$ when control embryos reached mid-gastrula and mid-tailbud stage. See also 
bioRxiv preprint doi: https://doi.org/10.1101/306803; this version posted June 3, 2019. The copyright holder for this preprint (which was not certified by peer review) is the author/funder, who has granted bioRxiv a license to display the preprint in perpetuity. It is made available under aCC-BY 4.0 International license.

423 Supplementary Movie 1. (b) Phenotypical rescue of mPouV/Sox3 LOF embryos by the co-injection of both $X$. laevis

424 Pou5f3.3 and Sox 2 mRNA alongside $m$ Cherry mRNA as a tracer. (c) Experimental design: Profiling the poly(A) RNA

425 transcriptome $(n=3)$ over three consecutive developmental stages under indicated conditions. Abbreviations used for the

426 developmental timeline: MBT, midblastula transition; 1B, late blastula; and eG, early gastrula. (d) Biplot of PC1 (accounting

427 for $86 \%$ variance) and PC2 (8\% variance) shows the relationship of developmental stage-specific poly(A) RNA

428 transcriptomes of control and $\alpha$-amanitin-injected embryos in biological triplicates (\#1-3). (e) Detection of 3,687 zygotic

429 genes with reduced transcript levels $(\geq 50 \%$, FDR $\leq 10 \%)$ in $\alpha$-amanitin-injected embryos. These genes were used as

430 reference for all other LOFs. Dots in scatterplot are colored according to the maternal contribution ${ }^{50}$ to the transcript level of

431 each of these zygotic genes. (f) Scatterplot of transcript fold changes (FCs) caused by mPouV and mPouV/Sox3 LOFs with

432 dots colored according to the ratio of FCs. Numbered dots refer to some developmentally relevant genes listed in (e). (g)

433 Early gastrula-staged WMISH: mir427, animal view; ventx, lateral view, ventral side facing right; and tbxt, dorsal view.

434 Numbers in the right bottom corner of each image refer to the count of embryos detected with the displayed WMISH

435 staining among all embryos analyzed per condition and in situ probe. Scale bars, $0.5 \mathrm{~mm}$. 
bioRxiv preprint doi: https://doi.org/10.1101/306803; this version posted June 3, 2019. The copyright holder for this preprint (which was not certified by peer review) is the author/funder, who has granted bioRxiv a license to display the preprint in perpetuity. It is made available under aCC-BY 4.0 International license.

a

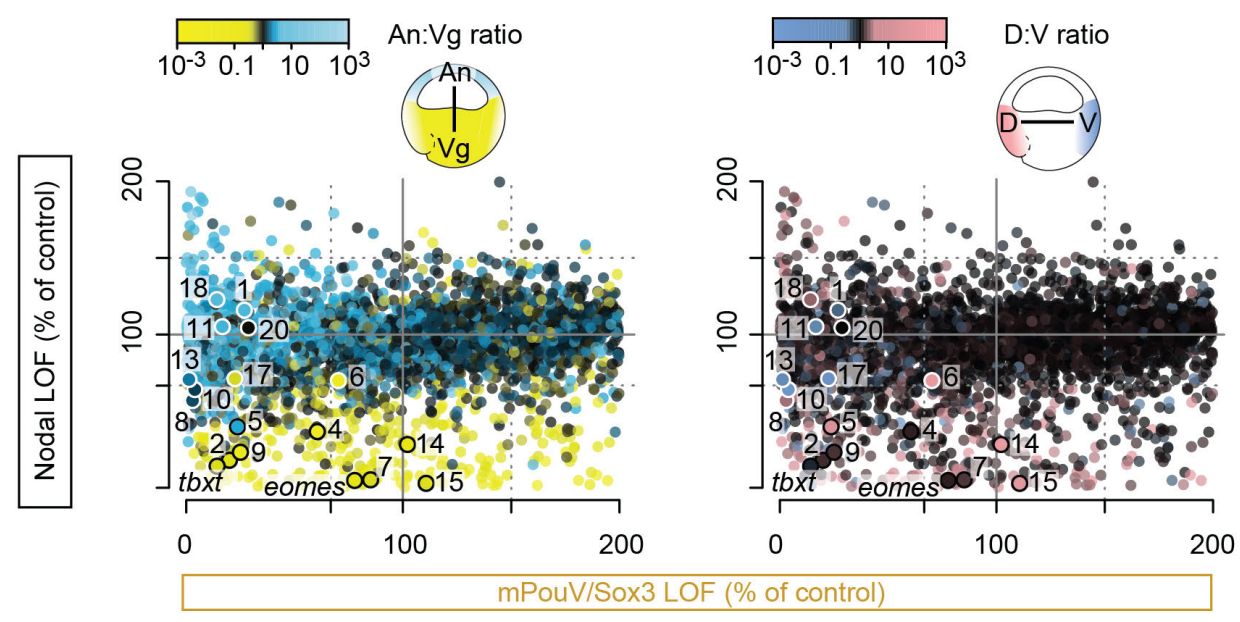

b

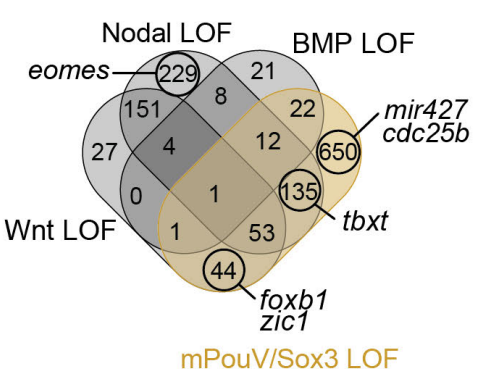

C

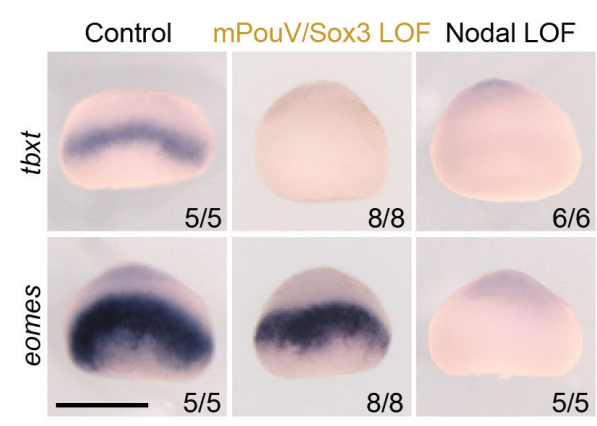

d

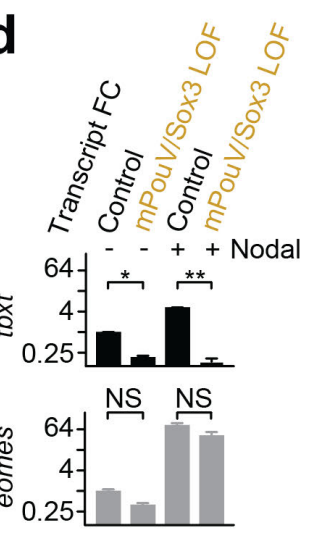

e

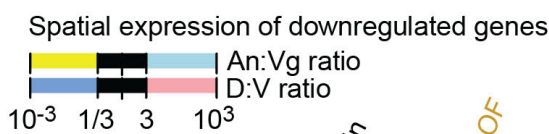

$\begin{array}{llll}10^{-3} & 1 / 3 & 3 & 10^{3}\end{array}$
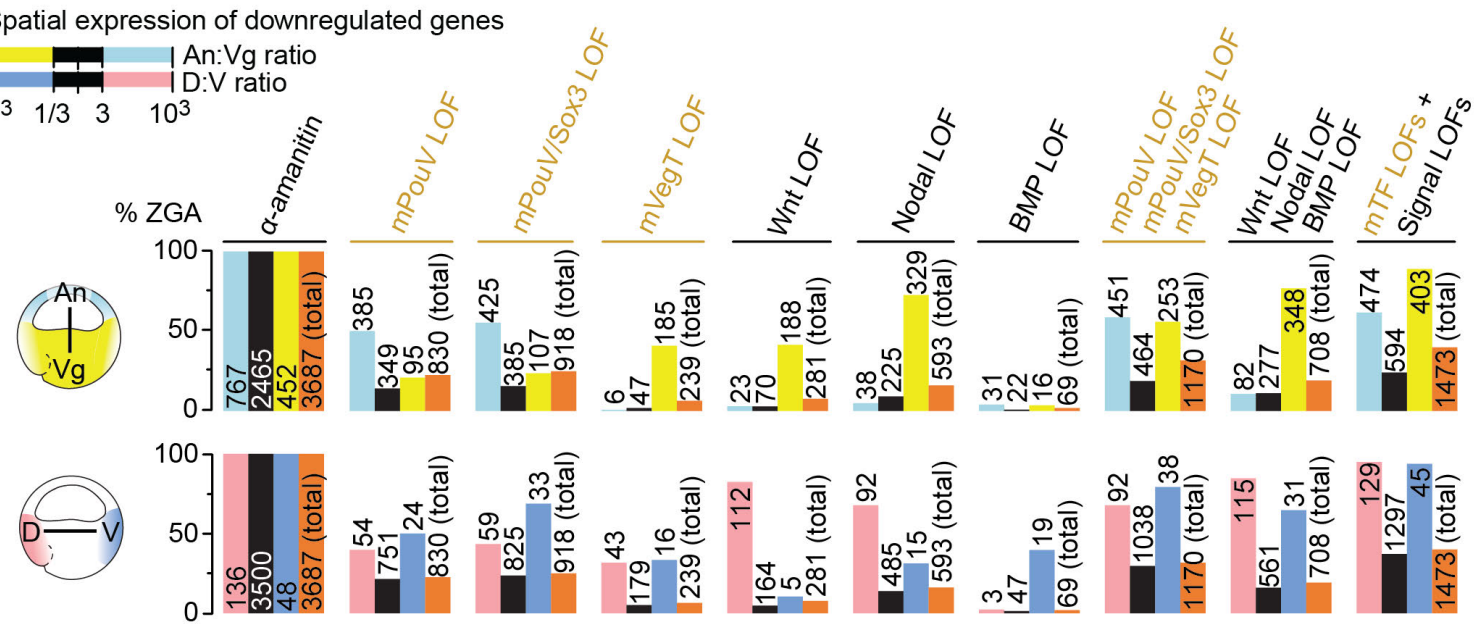

Figure 7 Signal-induced regionalization of ZGA depends on maternal TFs. (a) Transcriptional comparison of zygotic genes between the LOFs of maternal PouV/Sox3 and Nodal signaling. Dots are colored according to the normal ratio of transcript levels (regional expression) across the animal-vegetal (An: $\mathrm{Vg}$ ) or dorso-ventral (D:V) axis ${ }^{51}$. Numbered dots refer to genes listed in Fig. 6e. (b) Venn diagram of genes downregulated by the mPouV/Sox3 LOF (orange) or the LOF of single signal transduction pathways (black). (c) Early gastrula-staged WMISH for tbxt and eomes under indicated LOFs. Scale bar, $0.5 \mathrm{~mm}$. (d) Quantification of tbxt and eomes transcript levels in control and mPouV/Sox3 LOF animal caps with or without stimulated Nodal signaling. Error bars, mean+SD $(n=2)$. Two-tailed Student's t-test: *, $\mathrm{p}=0.014 ; * *, \mathrm{p}=0.005$; and NS, not significant $(\mathrm{p} \geq 0.02)$. (e) Bar graphs show the percentage (and number) of downregulated zygotic genes (\% ZGA under indicated LOFs) grouped by the normal ratio of transcript levels (regional expression) across the animal-vegetal (An: $\mathrm{Vg}$ ) and dorso-ventral (D:V) axis. 
bioRxiv preprint doi: https://doi.org/10.1101/306803; this version posted June 3, 2019. The copyright holder for this preprint (which was

not certified by peer review) is the author/funder, who has granted bioRxiv a license to display the preprint in perpetuity. It is made available under aCC-BY 4.0 International license.

a

MBT vs. MBT $\quad \begin{aligned} & \text { Differential chromatin profiling } \\ & \text { - Chromatin accessilibity (DNase-Seq) }\end{aligned}$

- Control m mPouV/Sox3 LOF - H3K4me1, RNAPII, Smad2 and $\beta$-catenin (ChIP-Seq)

b

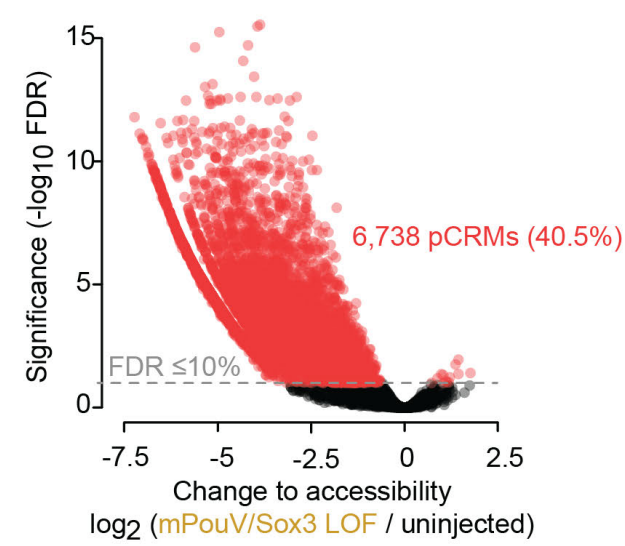

C

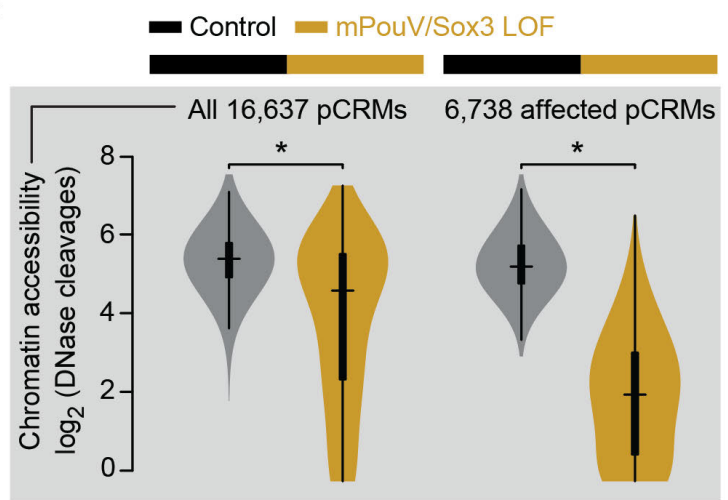

d

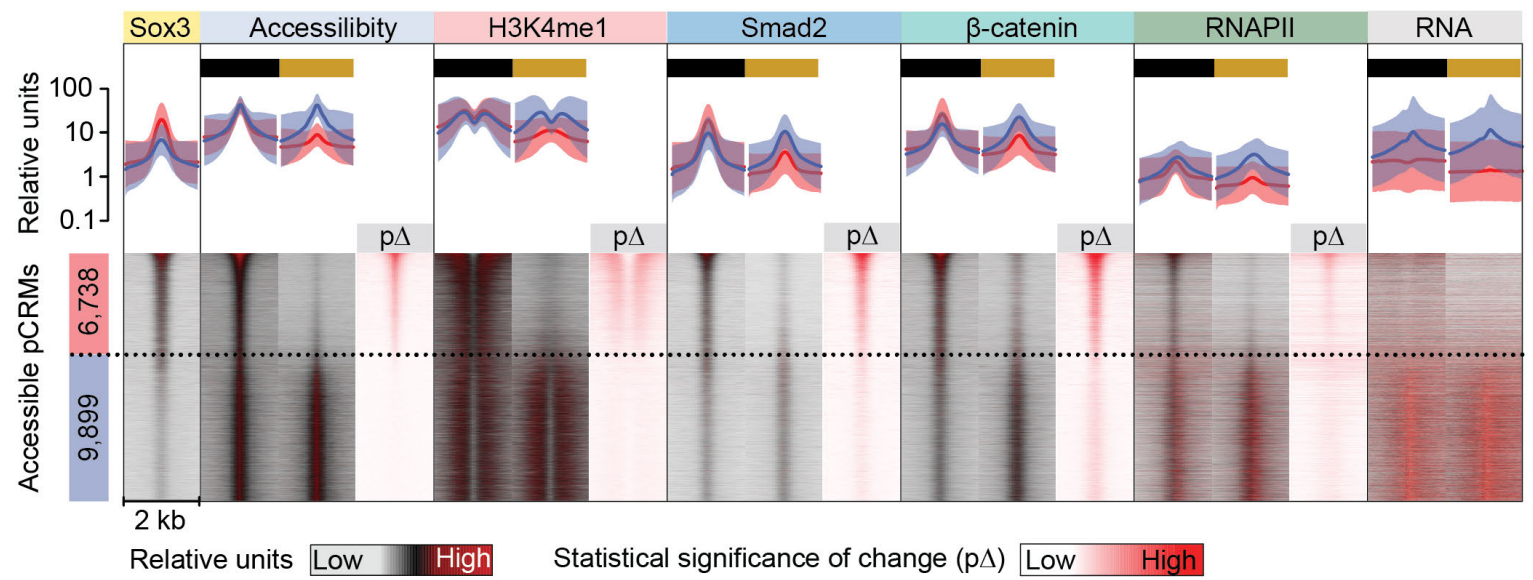

e

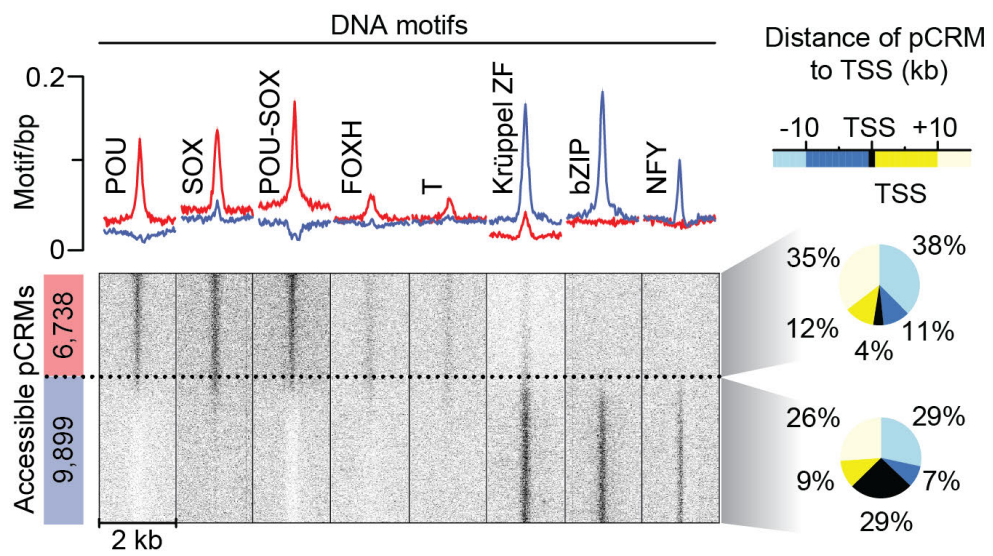

g mPouV/Sox3 LOF vs control pCRM accessibility $\rightarrow$ Gene activation unaffected $\begin{array}{lllll}\text { reduced } & 150 \%\end{array}$ (FDR $\leq 10 \%) \quad \overline{\text { FDR }}$

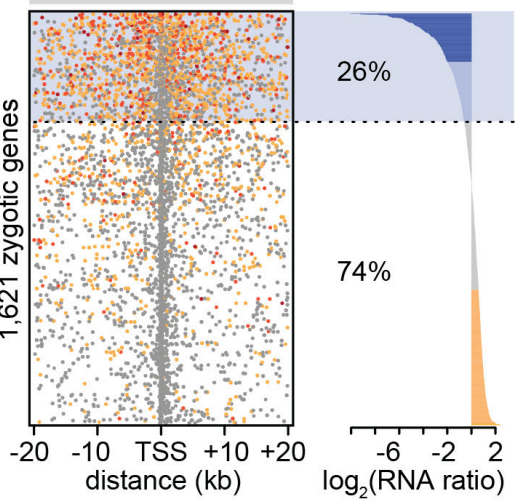

Figure 8 Maternal pluripotency TFs mPouV and Sox3 remodel 40\% of the accessible chromatin landscape to contribute to one quarter of ZGA. (a) Used approach to reveal the effect of mPouV/Sox3 on chromatin accessibility (DNase-Seq; $n=2$ ) and chromatin composition (ChIP-Seq; $\mathrm{n}=2$ ) at MBT. (b) Double-logarithmic volcano plot shows massive chromatin accessibility 
bioRxiv preprint doi: https://doi.org/10.1101/306803; this version posted June 3, 2019. The copyright holder for this preprint (which was not certified by peer review) is the author/funder, who has granted bioRxiv a license to display the preprint in perpetuity. It is made available under aCC-BY 4.0 International license.

$452 \leq 10 \%$ ) are marked in red. (c) Violin plots show the comparison of chromatin accessibility (DNase cleavages) between 453 uninjected and mPouV/Sox 3 LOF embryos at all and affected (FDR $\leq 10 \%$ ) pCRMs. Wilcoxon test: *, p $<2.2 \times 10^{-16}$. (d) 454 Normalized meta-plots (top row, mean \pm SD) and heat maps (bottom row) show the level of chromatin accessibility, chromatin 455 engagement (Sox3, H3K4me1, $\beta$-catenin, Smad2 and RNAPII) and RNA ( $n=3)$ across accessible pCRMs in uninjected and $456 \mathrm{mPouV} / \mathrm{Sox} 3 \mathrm{LOF}$ embryos. The $\beta$-catenin binding profile was generated at late blastula stage rather than the 1,024-cell stage 457 (Sox3) or MBT (all others). RNA was profiled at and beyond MBT as shown in Fig. 6c. In the heat map, the pCRMs are sorted 458 and grouped by significantly reduced DNase cleavages under mPouV/Sox 3 LOF: red group, affected (FDR $\leq 10 \%)$ and blue 459 group, unaffected (FDR $>10 \%$ ). These groups are represented in the meta-plots. Each heat map under mPouV/Sox $3 \mathrm{LOF}$ are 460 followed by a heat map showing the statistical significance of changes $(\mathrm{p} \Delta$ ) caused by mPouV/Sox 3 LOF. (e) Heat map shows 461 the occurrence of DNA motifs at accessible pCRMs sorted and grouped as in (d). (f) Pie charts summarize the distribution of 462 distances $(\mathrm{kb})$ to nearest zygotic TSSs of affected (top pie chart) and unaffected (bottom pie chart) pCRMs. (g) Panel compares 463 the effect of mPouV/Sox3 LOF on chromatin accessibility and RNAPII-mediated gene expression. Plot to the left shows the 464 localization of accessible pCRMs (affected, dot colored in orange to red with FDR decreasing from 10\%; and unaffected, grey 465 dot) relative to the zygotic TSSs that are active by the $\mathrm{MBT}^{52}$ and produce enough RNA transcripts to show significant $\geq$ two466 fold reductions upon $\alpha$-amanitin injection (Fig. 6e). Gene loci are sorted by mPouV/Sox3 LOF-induced transcript fold changes 467 as shown in the log-scaled bar graph to the right. 


\section{a}

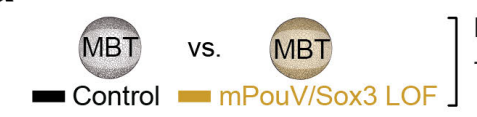

b

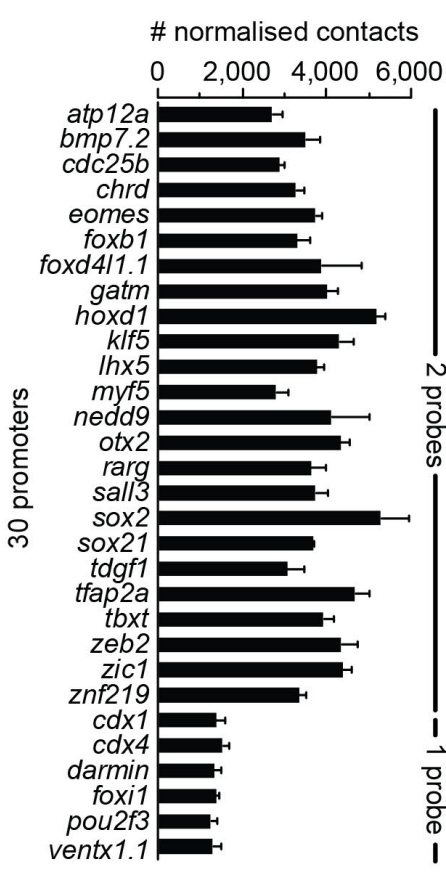

C

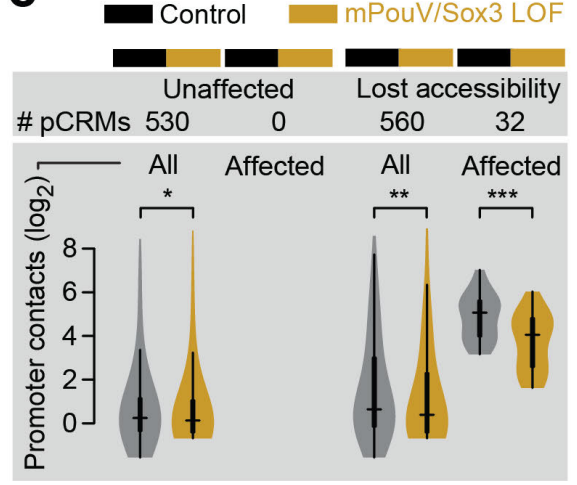

Differential chromatin profiling pCRM contacts with 30 promoters (next-generation capture-C)

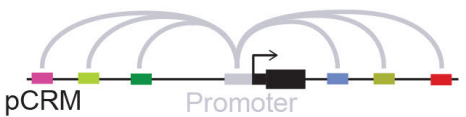

$$
\text { d - Control }-\mathrm{mPouv/So \times 3} \mathrm{LOF}
$$
Statistical significance of change $(\mathrm{p} \Delta)$ Low High
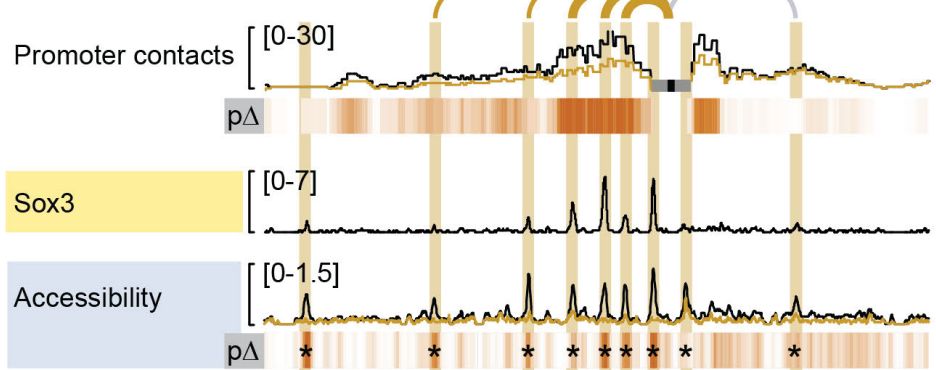

Smad2

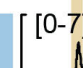

7]<smiles></smiles>

$\mathrm{p} \Delta$

$\beta$-catenin

$[0-6]$

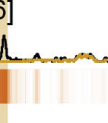<smiles>[Mg][Mg][Mg]</smiles>

$\mathrm{p} \Delta$

H3K4me1

[0-2]

$\mathrm{p} \Lambda$

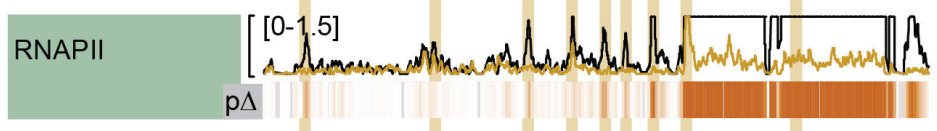

RNA
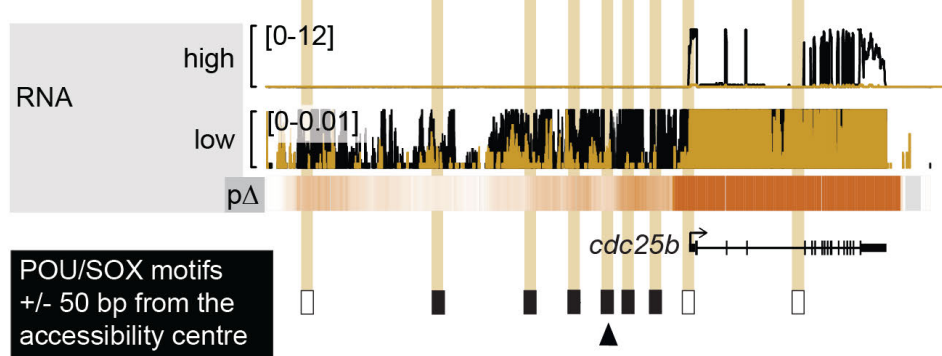

Figure 9 Pioneering activity of $\mathrm{mPouV} / \mathrm{Sox} 3$ initiates extensive chromatin remodeling such as the chromatin looping of distal pCRMs with promoters. (a) Used approach to reveal the effect of $\mathrm{mPouV} / \mathrm{Sox} 3$ on chromatin conformation between 30 promoters and distal pCRMs (next-generation capture-C; $n=3$ ) at MBT. (b) Bar graph shows the number of normalized contacts (mean+SD; $n=6$ ) derived from non-redundant capture-reporter FLASH ${ }^{53}$ reads (Supplementary Fig. 12b) for each promoter captured with one or two probes (Supplementary Table 10). (c) Violin plots compare the number of promoter contacts with accessible pCRMs between uninjected and mPouV/Sox3 LOF embryos. The comparison is stratified into pCRMs with stable and lost accessibility upon $\mathrm{mPouV} / \mathrm{Sox} 3 \mathrm{LOF}$ and shown for both all and affected (FDR $\leq 10 \%$ ) promoter contacts. Wilcoxon tests and effect size estimates: ${ }^{*}, \mathrm{p}=5 \times 10^{-5}$ and $\mathrm{r}_{\text {effect }}=0.12$ (small effect); ${ }^{* *}, \mathrm{p}=5 \times 10^{-30}$ and $\mathrm{r}_{\text {effect }}=0.34$ (medium effect); and ***, $\mathrm{p}=5 \times 10^{-10}$ and $\mathrm{r}_{\text {effect }}=0.78$ (large effect). (d) Superimposed line tracks show the level of promoter-tied chromatin conformations, chromatin accessibility and various chromatin components (Smad2, $\beta$-catenin, H3K4me1 and RNAPII) at the $c d c 25 b$ gene locus between control (uninjected) and mPouV/Sox3 LOF embryos. The RNA track is split into a high (0-12) and low (0-0.01) expression window. Note that the low-expression window shows that locally transcribed noncoding super-enhancer RNA depend on $\mathrm{mPouV} / \mathrm{Sox} 3$ as well as the gene $c d c 25 b$. Heat maps $(\mathrm{p} \Delta)$ below each superimposed line plot show the statistical significance of changes caused by mPouV/Sox3 LOF. The footer highlights the occurrences of canonical POU/SOX motifs (black filled rectangles) at accessible pCRMs ( \pm 50 bp from the accessibility centre) and one 
bioRxiv preprint doi: https://doi.org/10.1101/306803; this version posted June 3, 2019. The copyright holder for this preprint (which was not certified by peer review) is the author/funder, who has granted bioRxiv a license to display the preprint in perpetuity. It is made available under aCC-BY 4.0 International license.

484 strongly affected pCRM with an arrowhead. Asterisks on the $\mathrm{p} \Delta$ heat map mark significant (FDR $\leq 10 \%$ ) reductions to $\mathrm{pCRM}$

485 accessibility. pCRMs are boxed in and their frequency of contacts with the $c d c 25 b$ promoter are illustrated with an arc of varying strength. Boxes of affected pCRM and arcs of promoter contacts are colored orange. 
bioRxiv preprint doi: https://doi.org/10.1101/306803; this version posted June 3, 2019. The copyright holder for this preprint (which was

not certified by peer review) is the author/funder, who has granted bioRxiv a license to display the preprint in perpetuity. It is made available under aCC-BY 4.0 International license.

a

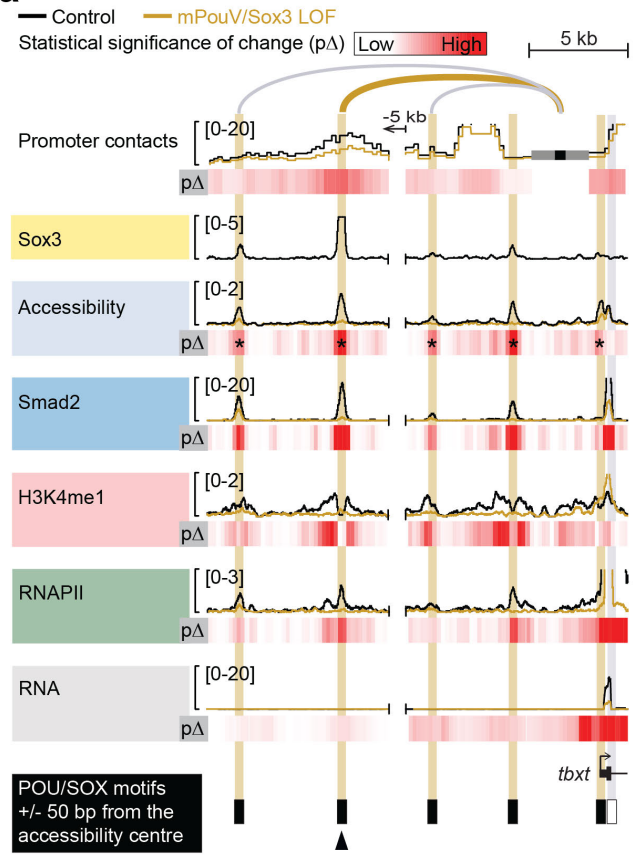

b

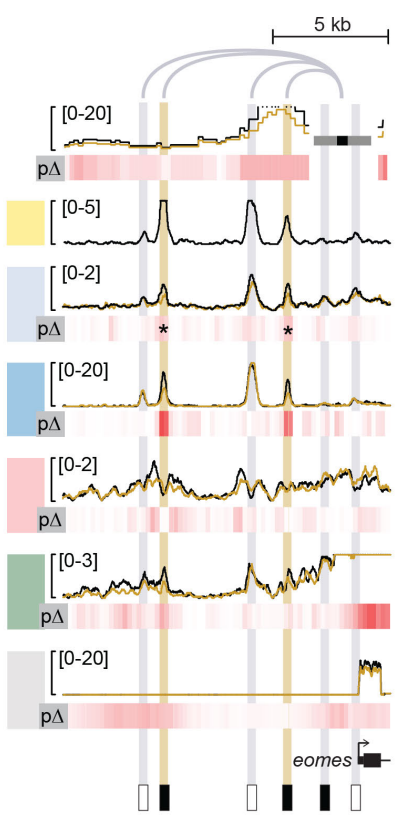

C

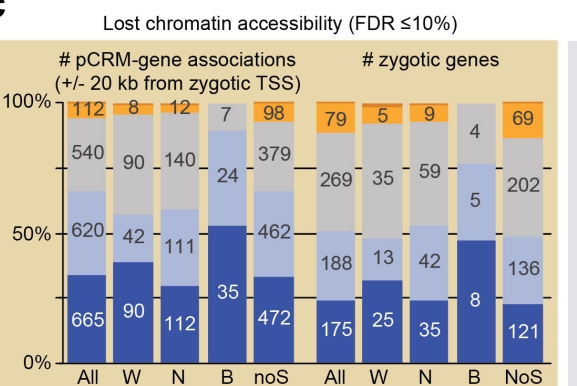

Unaffected chromatin accessibility (FDR $\leq 10 \%$ ) \# zygotic genes $(+/-20 \mathrm{~kb}$ from zygotic TSS)

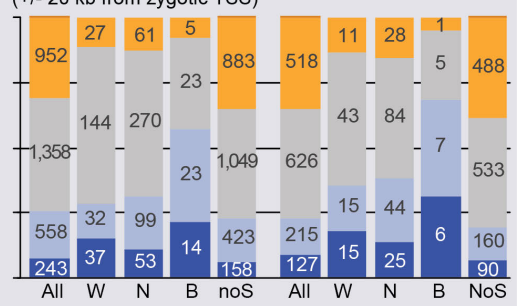

RNA: MPouV/SOX3 LOF vs control
$0 \quad 25 \quad 67150400 \%$

All: all zygotic genes

$\mathrm{W}:$ Wnt-responsive genes

$\mathrm{N}$ : Nodal-responsive genes

B: Bmp-responsive genes

NoS: Wnt/Nodal/Bmp-independent genes

d

All Xenopus embryonic cells before inductive signalling (pre-MBT)

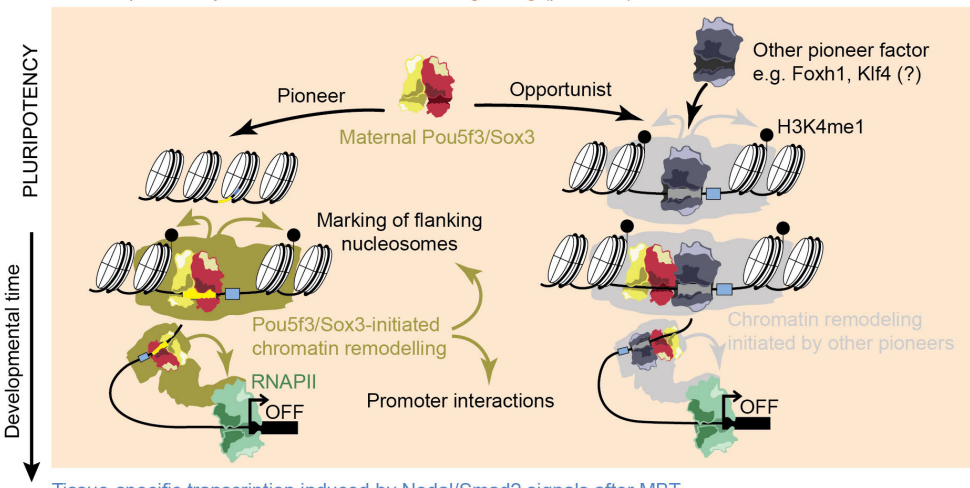

Tissue-specific transcription induced by Nodal/Smad2 signals after MBT

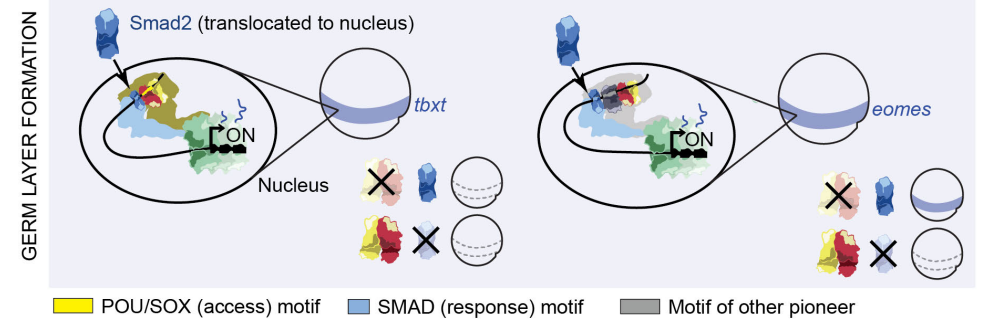


bioRxiv preprint doi: https://doi.org/10.1101/306803; this version posted June 3, 2019. The copyright holder for this preprint (which was not certified by peer review) is the author/funder, who has granted bioRxiv a license to display the preprint in perpetuity. It is made available under aCC-BY 4.0 International license.

488 Figure 10 Maternal Pou5f3/Sox3-initiated chromatin remodeling to prime the first transcriptional response to inductive signals during ZGA. (a,b) Superimposed line tracks show the level of promoter-tied chromatin conformations, chromatin accessibility and various chromatin components (Smad2, H3K4me1 and RNAPII) at the Nodal-responsive mesoderm determinants $t b x t$ (a) and eomes (b) between control (uninjected) and mPouV/Sox3 LOF embryos. Heat maps ( $\mathrm{p} \Delta$ ) below each superimposed line track show the statistical significance of changes caused by mPouV/Sox3 LOF. The footer highlights the occurrences of canonical POU/SOX motifs (black filled rectangles) at accessible pCRMs ( $\pm 50 \mathrm{bp}$ from the accessibility centre) and one strongly affected pCRM with an arrowhead. Asterisks on the $\mathrm{p} \Delta$ heat map mark significant (FDR $\leq 10 \%)$ reductions to pCRM accessibility. pCRMs are boxed in and their frequency of contacts with the promoter are illustrated with an arc of varying strength. Boxes of affected pCRM and arcs of promoter contacts are colored orange. (c) Stacked bar graphs summarize the correlation of unaffected and significantly reduced (FDR $\leq 10 \%$ ) pCRM accessibility with RNAPII-mediated expression of all, signal responsive and non-responsive zygotic genes in mPouV/Sox3 LOF embryos at MBT. These correlations and corresponding numbers (placed on the stacked bars) are shown for pCRM-gene associations and zygotic genes. TSS-centric maps of reduced chromatin accessibility are shown for signal responsive and non-responsive genes in Supplementary Figs. 15-17. (d) Model of chromatin pioneering and opportunistic engagement to predefine first zygotic responses to inductive signals. 
bioRxiv preprint doi: https://doi.org/10.1101/306803; this version posted June 3, 2019. The copyright holder for this preprint (which was not certified by peer review) is the author/funder, who has granted bioRxiv a license to display the preprint in perpetuity. It is made available under aCC-BY 4.0 International license.

a Counts of pCRMs detected by RNAPII ChIP-Seq Developmental time

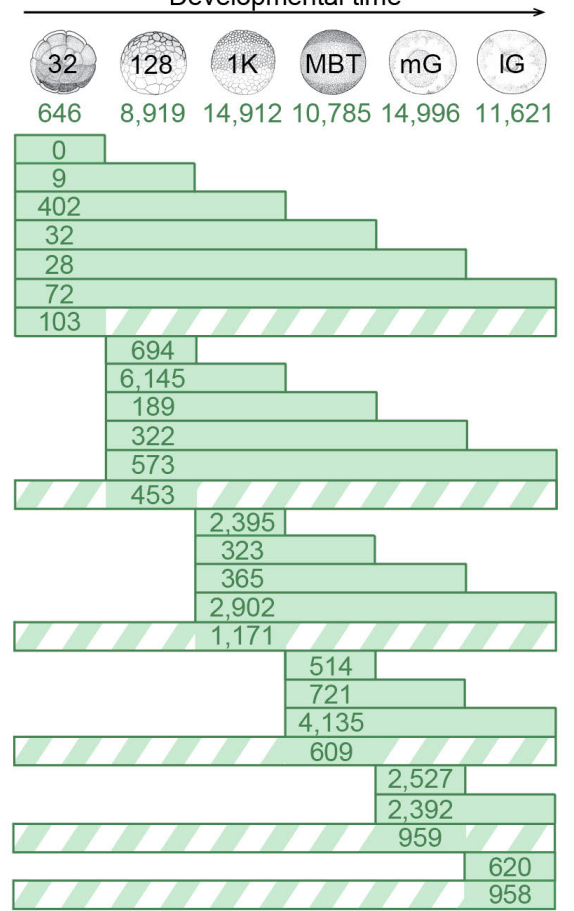

C

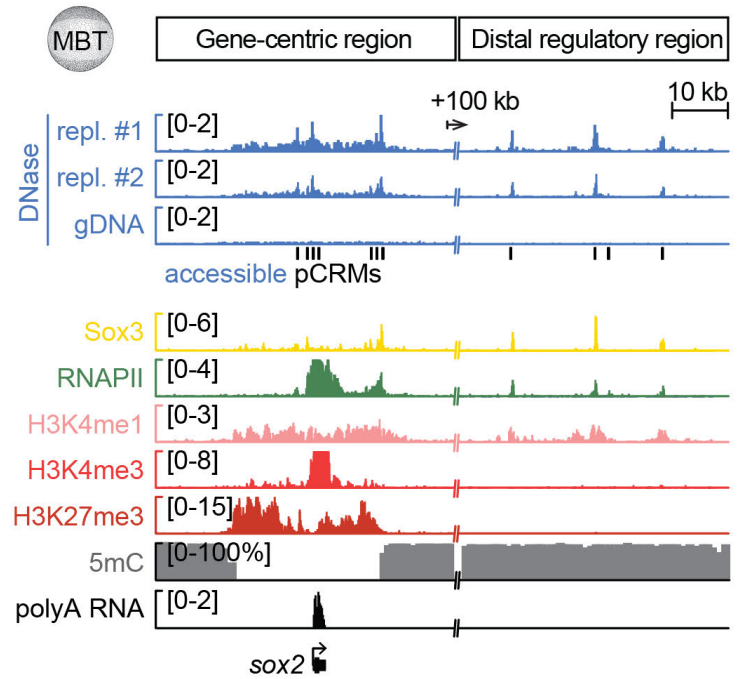

b
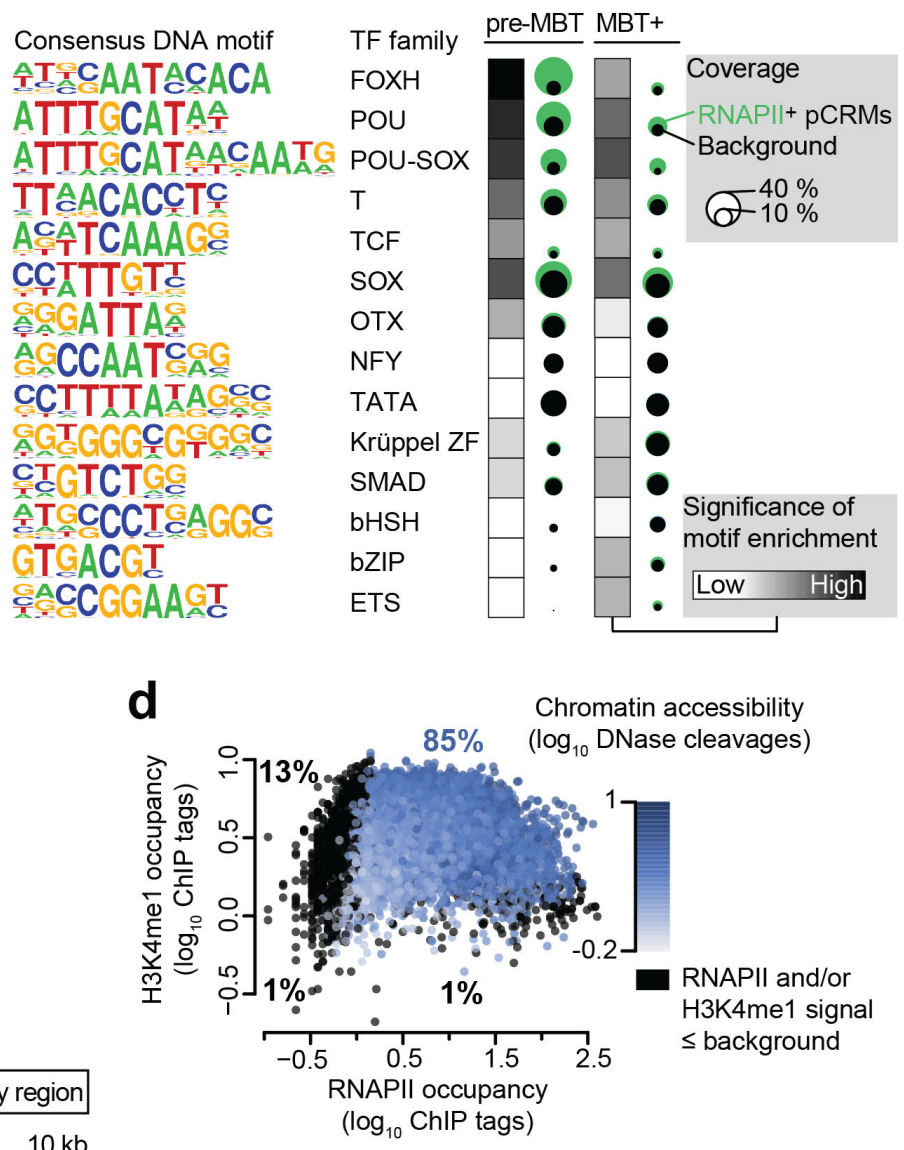

e

pCRMs detected by RNAPII ChIP-Seq

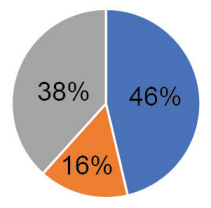

pCRMs detected by DNase-Seq

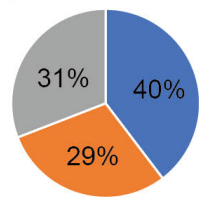

Intergenic

Promoter-TSS

Genebody

Supplementary Figure 1 Characterization of pCRMs instructing ZGA. (a) Graph shows the temporal dynamics of RNAPIIengaged $\left(\mathrm{RNAPII}^{+}\right)$pCRMs ( $\geq 1 \mathrm{ChIP}$ tag/million) from the 32-cell to the late gastrula stage. Striped bars refer to RNAPII ${ }^{+}$ pCRMs fragmentarily detected across consecutive developmental stages. Abbreviations used for the developmental timeline: 32, 128 and $1 \mathrm{~K}, 32-, 128$ - and 1,024-cell stage; MBT, mid-blastula transition; mG and 1G, mid- and late gastrula stage. (b) Heat map and bubble plot show the respective statistical significance and coverage of DNA motifs enriched among the top 5,000 RNAPII $^{+}$pCRMs detected from the 32-cell to the 1,024-cell stage (pre-MBT) and from the MBT to the late gastrula stage $(\mathrm{MBT}+)$. This analysis included 39,785 (pre-MBT) and 41,898 (MBT+) genomic 'background' regions matching the overall GC contents of the selected RNAPII ${ }^{+}$pCRMs. (c) Validation of our DNase-Seq method: DNase-probed chromatin accessibility (biological replicates \#1 and \#2) at the sox2 locus (gene-centric region and downstream distal regulatory region) is shown alongside with various other chromatin features (Sox3, RNAPII, H3K4me1, poly(A) RNA from this study and 
bioRxiv preprint doi: https://doi.org/10.1101/306803; this version posted June 3, 2019. The copyright holder for this preprint (which was not certified by peer review) is the author/funder, who has granted bioRxiv a license to display the preprint in perpetuity. It is made available under aCC-BY 4.0 International license.

515 H3K4me3, H3K27me3, 5-methylcytosine [5mC] from ref. ${ }^{54}$ ) detected around the MBT. DNase-treated naked genomic DNA

516 (gDNA) was used as a negative control for DNase-probed chromatin accessibility. (d) Biplot shows the DNA occupancy levels

517 of RNAPII and H3K4me1 at accessible pCRMs. pCRMs (dots) are colored according to their chromatin accessibility except

518 for the pCRMs that showed RNAPII and/or H3K4me1 signals piled up over a distance of $1 \mathrm{~kb}$ below background. (e) Pie

519 charts show the genomic distribution of pCRMs detected by RNAPII ChIP-Seq and DNase-Seq, respectively. 
bioRxiv preprint doi: https://doi.org/10.1101/306803; this version posted June 3, 2019. The copyright holder for this preprint (which was

not certified by peer review) is the author/funder, who has granted bioRxiv a license to display the preprint in perpetuity. It is made available under aCC-BY 4.0 International license.

a

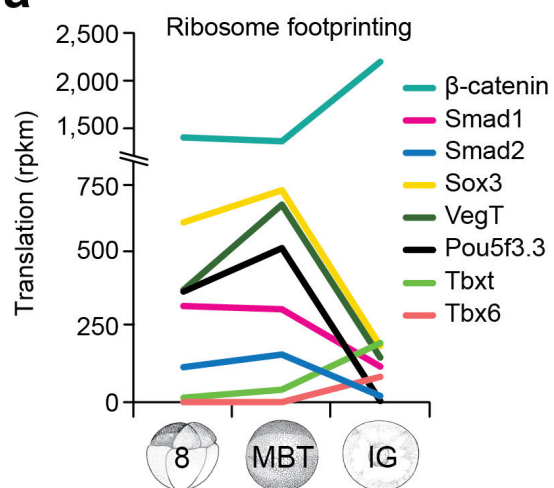

e

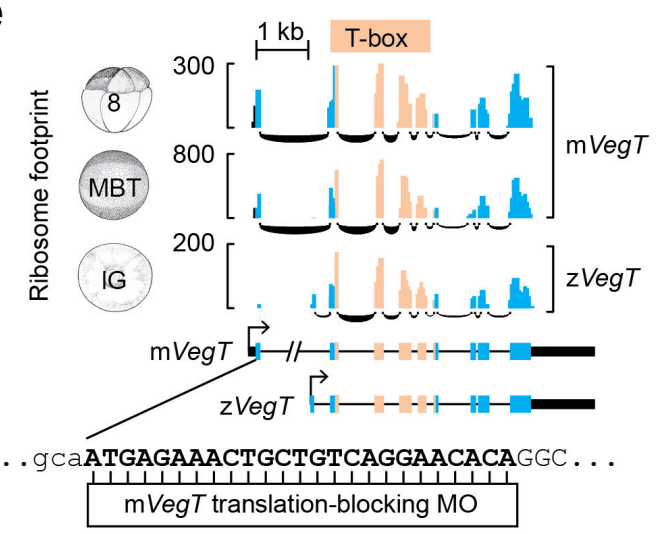

h

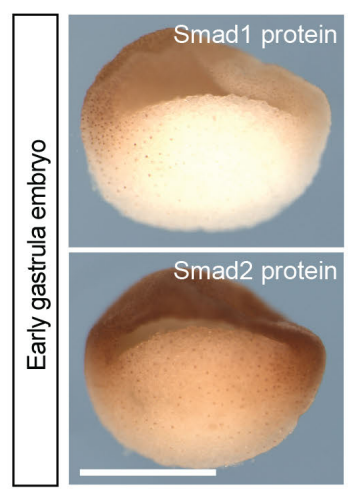

k

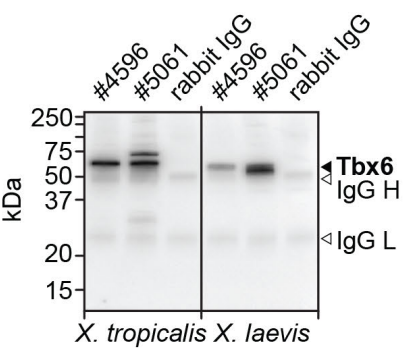

I

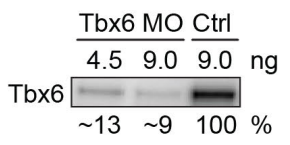

a-tubulin b

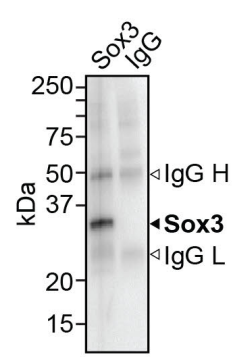

C

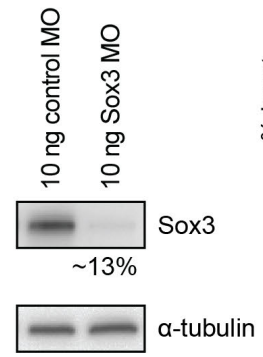

d

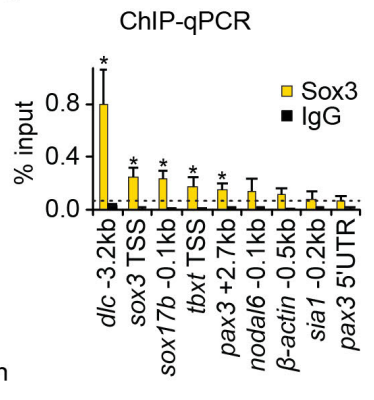

f

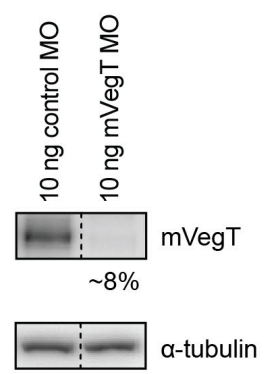

g

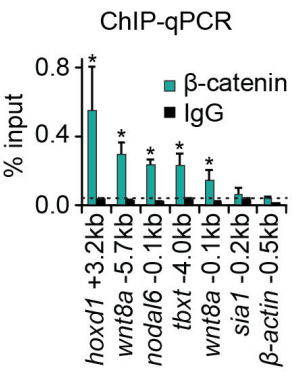

i

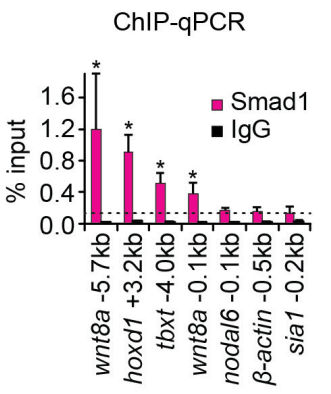

m

n

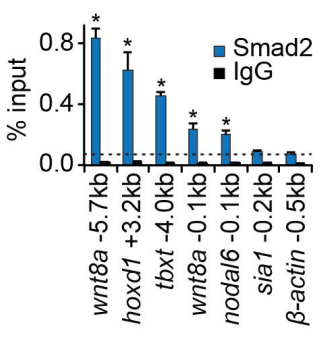

Supplementary Figure 2 Verification of antibodies against frequently translated TFs and signal mediators. (a) Line chart shows the level of ribosome footprints ${ }^{13}$ of selected TFs and signal mediators across MBT in rpkm (reads per kilobase of 
bioRxiv preprint doi: https://doi.org/10.1101/306803; this version posted June 3, 2019. The copyright holder for this preprint (which was not certified by peer review) is the author/funder, who has granted bioRxiv a license to display the preprint in perpetuity. It is made available under aCC-BY 4.0 International license.

523 transcript per million mapped reads). Abbreviations used for the developmental timeline: 8, 8-cell stage; MBT, mid-blastula 524 transition; 1G, late gastrula stage. (b) Western blot shows the immunoprecipitation (IP) of Sox3 protein extracted from early 525 gastrula embryos. IgG H and L, detected IgG heavy and light chains of the IP antibody. (c) Western blot shows the level of 526 Sox3 protein immunoprecipitated from control (control MO) and Sox3-depleted (Sox3 MO) blastula embryos (Sox3 LOF). $\alpha$ 527 tubulin, IP input control. (d, g,i,j, ,n) Bar graphs show the ChIP-qPCR results as a percentage of ChIP input (mean+SD; n=2-3) 528 for Sox3, $\beta$-catenin, Smad1 and Smad2 at the early gastrula stage and for Tbx6 at the early neurula stage. One-tailed Student's 529 t-test (comparing to IgG control): *, $\mathrm{p} \leq 0.1$ and $\geq 2$-fold enrichment relative to the lowest DNA recovery with the ChIP antibody 530 (dotted line). (e) Ribosome footprinting tracks show the post-MBT switch of translation from the maternal (m) to the zygotic 531 (z) VegT transcript. The VegT translation-blocking MO was designed to block translation of the maternal transcript only. (f) 532 Western blot shows the level of mVegT protein immunoprecipitated from control (control MO) and mVegT-depleted (mVegT 533 MO) blastula embryos (mVegT LOF). Dotted line indicates the elimination of irrelevant lanes from the western blot. $\alpha$-tubulin, 534 IP input control. (h) WMIHC shows the spatial distribution of nuclear Smad1 and Smad2 protein on bisected early gastrula 535 embryos. Scale bar, $0.5 \mathrm{~mm}$. (k) Western blot shows the level of Tbx6 immunoprecipitated from X. tropicalis and X. laevis 536 late gastrula embryos. Antibody \#4596 was chosen for subsequent IP and ChIP experiments, and \#5061 for Western blotting. 537 (I) Western blot (WB) shows the level of Tbx6 protein immunoprecipitated from standard control and Tbx6 morphants at the 538 early tailbud stage. $\alpha$-tubulin, IP input control. (m) Line charts show the relative level of $t b x 6$ transcripts (RT-qPCR) and Tbx6 539 protein (IP/WB) from the early blastula to the early tailbud stage. $\alpha$-tubulin, IP input control. 
bioRxiv preprint doi: https://doi.org/10.1101/306803; this version posted June 3, 2019. The copyright holder for this preprint (which was

not certified by peer review) is the author/funder, who has granted bioRxiv a license to display the preprint in perpetuity. It is made available under aCC-BY 4.0 International license.

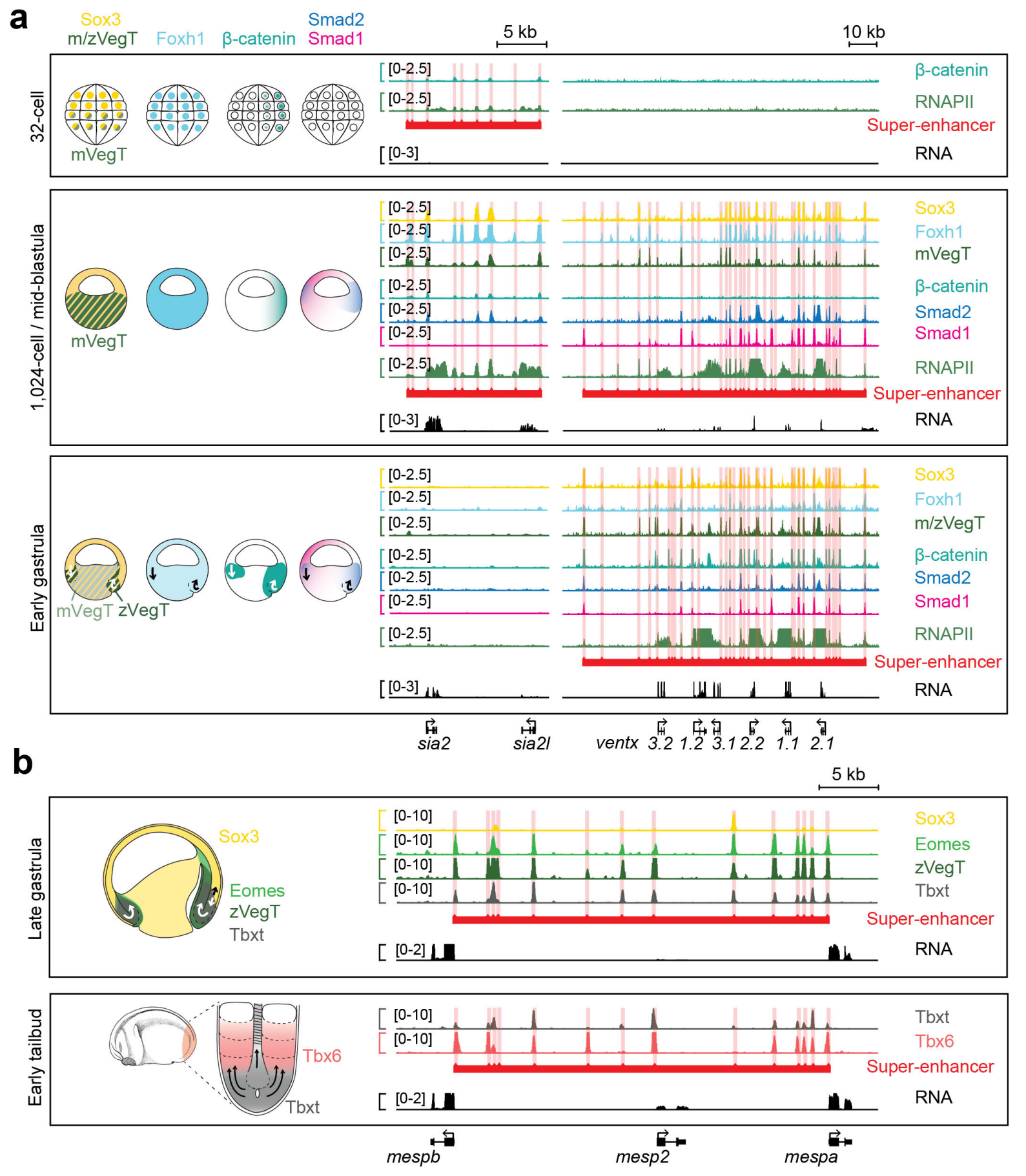

Supplementary Figure 3 Snapshots of TF and signal mediator binding to super-enhancers during early embryogenesis. (a)

542 Dynamic chromatin engagement of endogenous Sox3, Foxh1 ${ }^{18}$, VegT, $\beta$-catenin, Smad2, Smad1 and RNAPII to putative super-enhancers of the siamois and ventx gene cluster from the 32-cell to the early gastrula stage. Illustrated sagittal sections (dorsal side is right) show the nuclear localization of the selected TFs with arrows pointing to the tissue movements of gastrulation. (b) Snapshot of endogenous Sox3, Eomes, zVegT, Tbxt and Tbx6 binding to the putative super-enhancer of the mesp gene cluster at the late gastrula and/or early tailbud stage. Illustrated sagittal sections (dorsal side is right) show the nuclear localization of selected TFs in the late gastrula embryo and at the caudal end of the early tailbud embryo with arrows pointing to the tissue movements during axial elongation ${ }^{20}$. All super-enhancers were formed by stitching together engaged pCRMs that are $\leq 25 \mathrm{~kb}$ apart ${ }^{21}$. 
bioRxiv preprint doi: https://doi.org/10.1101/306803; this version posted June 3, 2019. The copyright holder for this preprint (which was

not certified by peer review) is the author/funder, who has granted bioRxiv a license to display the preprint in perpetuity. It is made available under aCC-BY 4.0 International license.

a
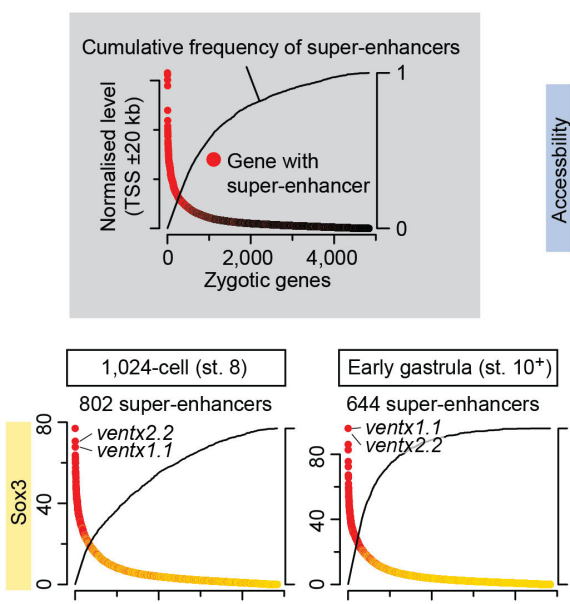
644 super-e
- ventx 1.1
ventx 2.2
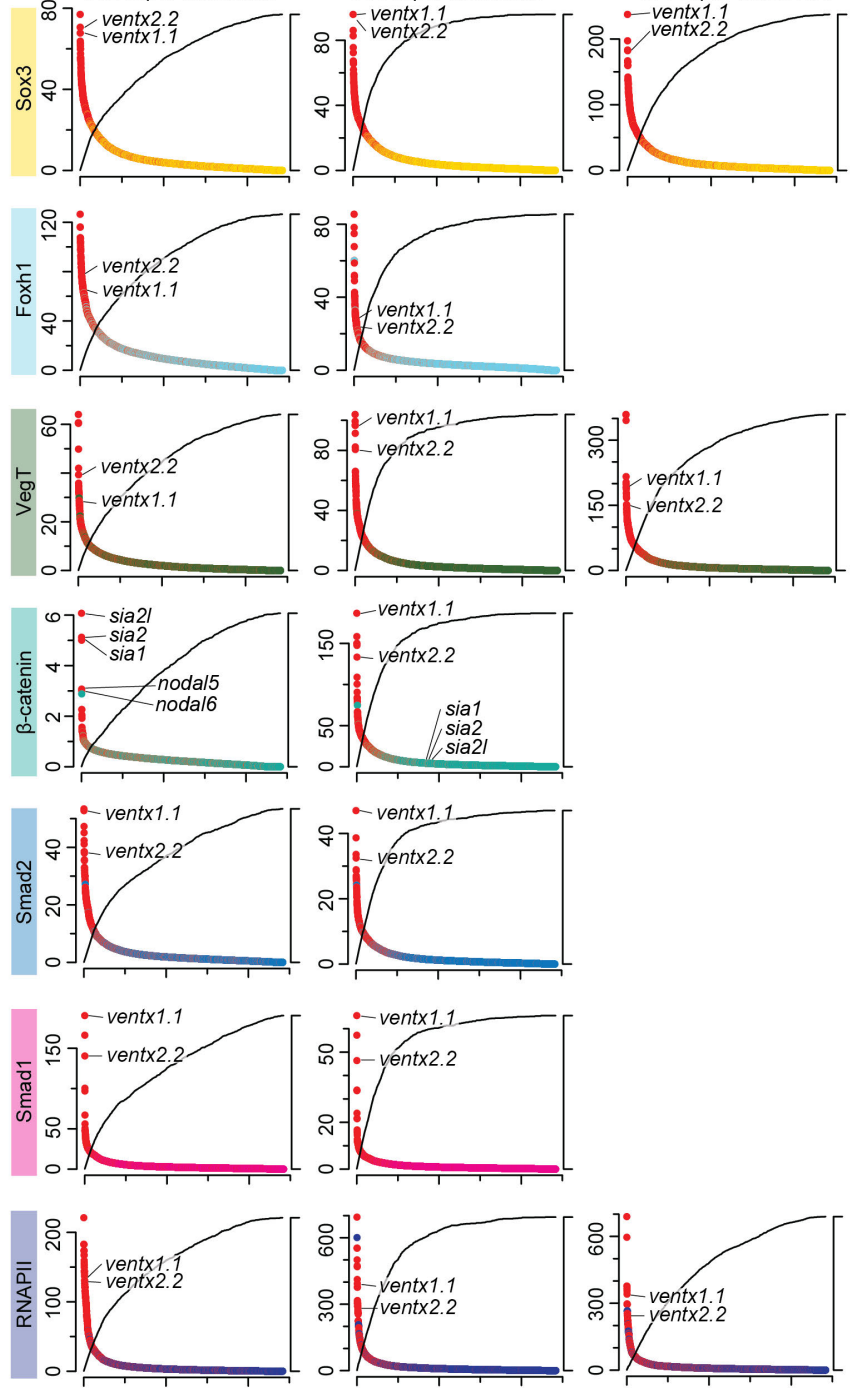

b
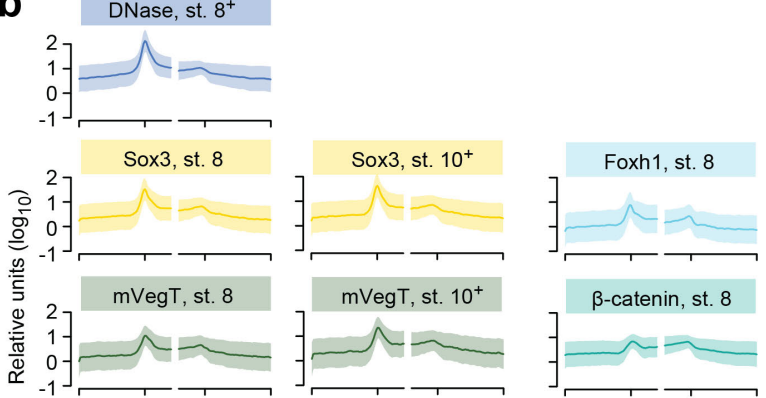

Foxh1, st. $10^{+}$
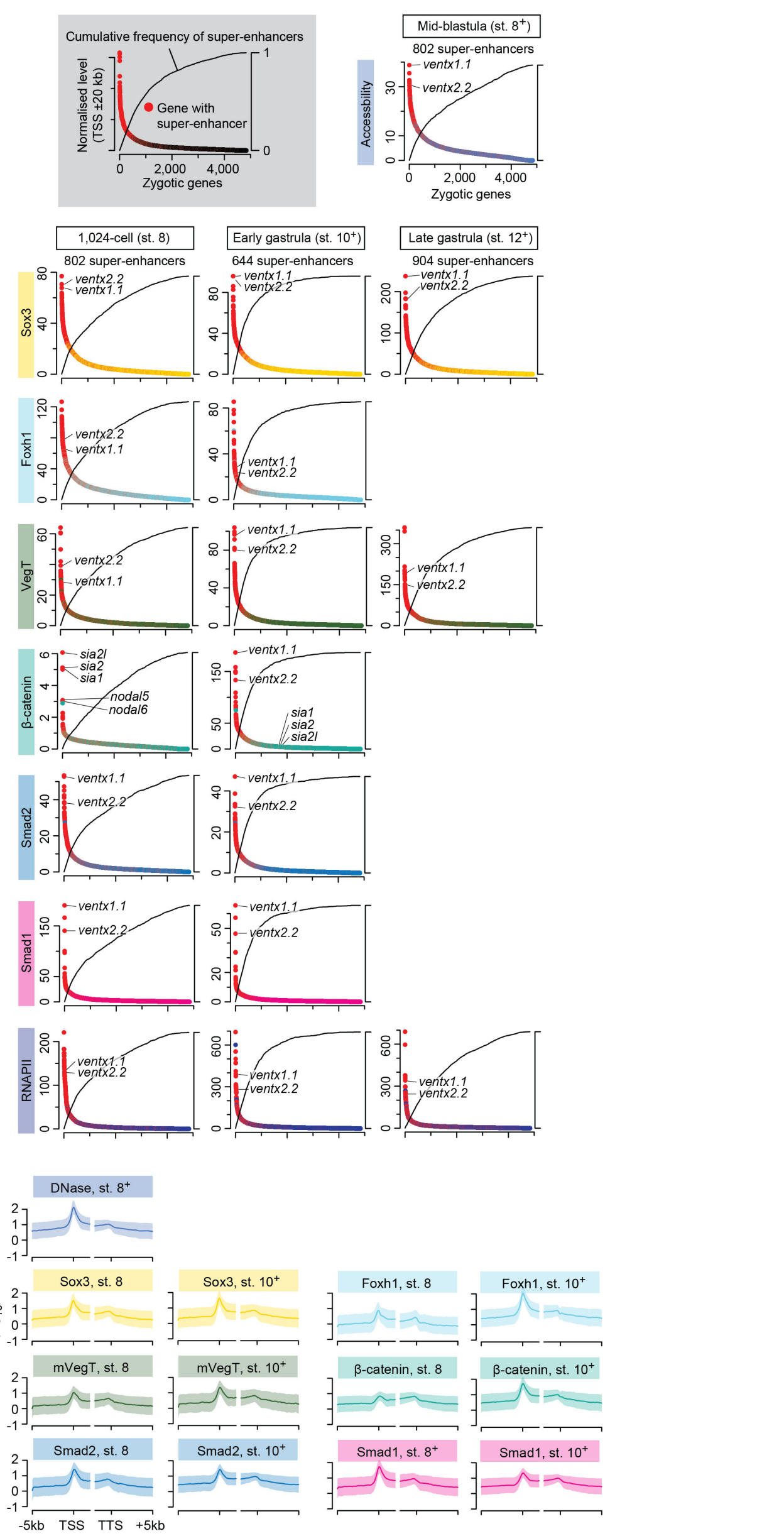
bioRxiv preprint doi: https://doi.org/10.1101/306803; this version posted June 3, 2019. The copyright holder for this preprint (which was not certified by peer review) is the author/funder, who has granted bioRxiv a license to display the preprint in perpetuity. It is made available under aCC-BY 4.0 International license.

551 Supplementary Figure 4 Characterization of genome-wide chromatin engagement and chromatin accessibility in early Xenopus embryos. (a) The grey box explains the composition of the following dot plots: the x-axis displays zygotic genes

553 (detected by RNAPII profiling from the 32-cell to the late gastrula stage ${ }^{52}$ ) ranked by the total level of pCRM accessibilities

554 or pCRM occupancies (normalized to 1 million mapped reads; primary y-axis) that are nearest ( $\leq 20 \mathrm{~kb}$ ) to corresponding 555 transcription start sites (TSSs). The secondary y-axis shows the cumulative frequency of gene-associated super-enhancers $(\leq 5$

$556 \mathrm{~kb}$ from TSSs). Genes with super-enhancers are highlighted in red. (b) Meta-summary (mean \pm SD; $\log _{10}$ scale) of chromatin 557 accessibility or DNA occupancy levels across zygotic TSSs and transcription termination sites (TTSs). For each developmental 558 stage, super-enhancers were formed by stitching together pCRMs that are $\leq 25 \mathrm{~kb}$ apart and engaged by RNAPII or at least one 559 TF or signal mediator. 
a

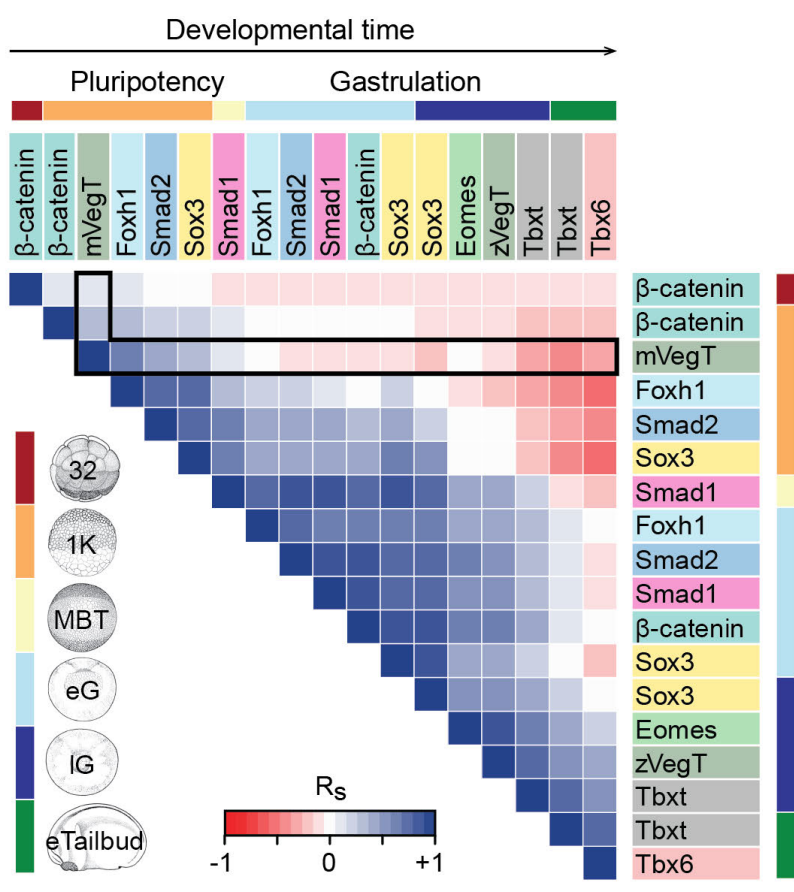

b

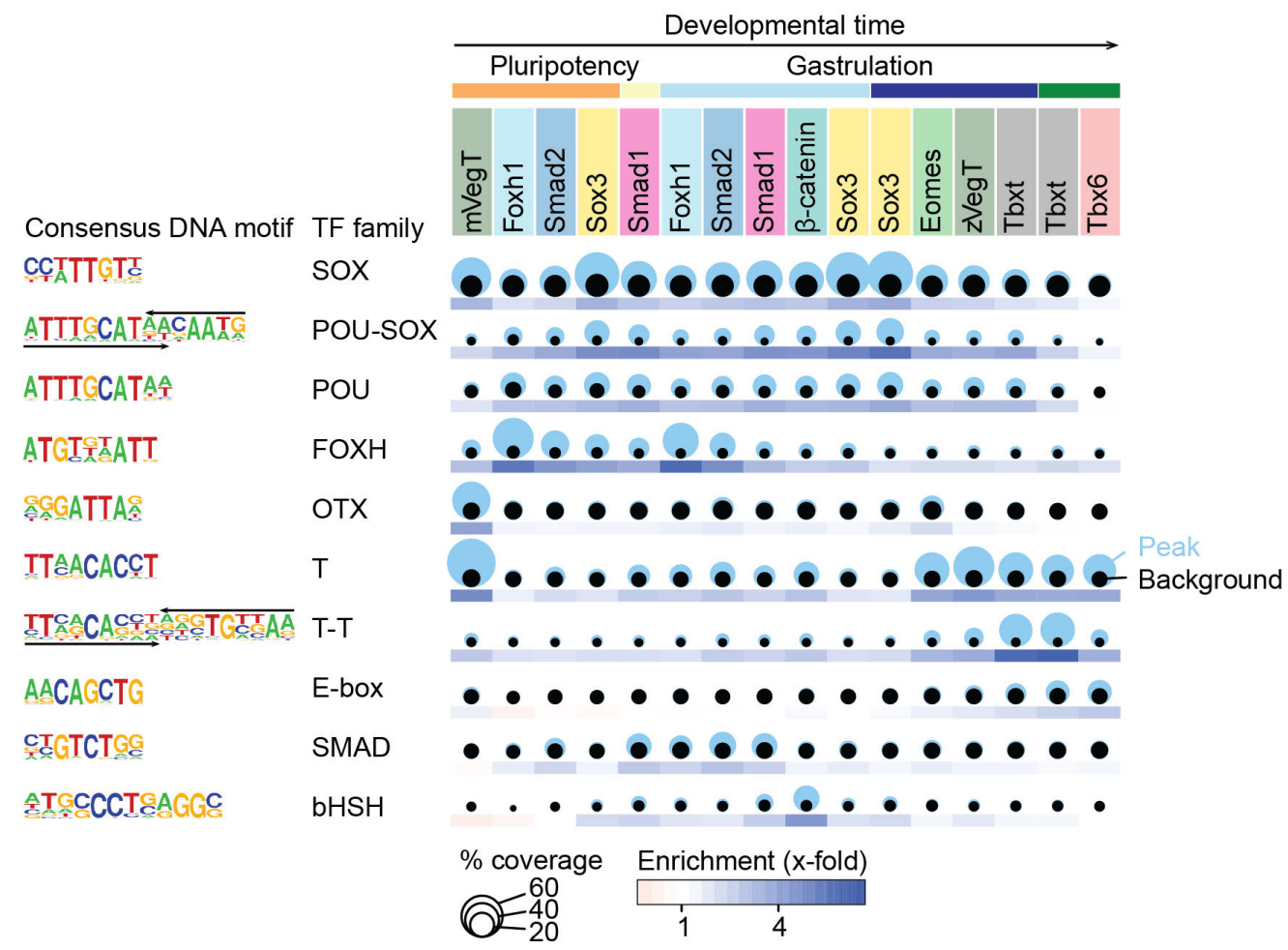

561 Supplementary Figure 5 The relationship of chromatin recruitment among TFs and signal mediators and its underlying preferences for specific DNA recognition motifs in pCRMs of pluripotent and neuro-mesodermal embryonic cells. (a) Heat map shows pairwise Spearman correlations $\left(\mathrm{R}_{\mathrm{s}}\right)$ of DNA occupancy levels at $\sim 12,500$ pCRMs among TFs (Sox3, mVegT, Foxh1 ${ }^{18,25}$, Eomes ${ }^{19}, \mathrm{zVegT}^{19}, \mathrm{Tbxt}^{19}$ and Tbx6) and signal mediators ( $\beta$-catenin, Smad1, Smad2) at indicated developmental stages. The pCRM coordinates were generated by collating the strongest 2,000 peaks of each binding profile. Abbreviations used for the developmental timeline: 32 and 1K, 32- and 1,024-cell stage; MBT, mid-blastula transition; eG and 1G, early and late gastrula stage; eTailbud, early tailbud stage. (b) Bubble plots and heat maps show the coverage and enrichment of DNA recognition motifs, respectively, among the top 2,000 pCRMs in each chromatin profile. 


$\underset{\text { Developmental time }}{\stackrel{\text { Gastrulation }}{\text { Pluripotency }}}$

DNA motifs

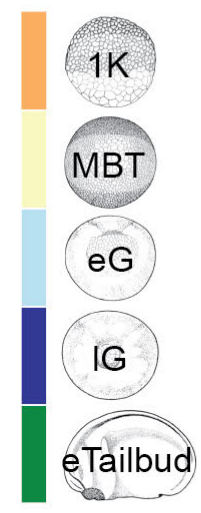

Supplementary Figure 6 pCRM binding patterns correlate with the occurrence of specific DNA motifs. Heat map to the left shows the relative DNA occupancy levels across $\sim 12,500$ pCRMs for a selection of TFs (mVegT, Sox3, Foxh $1^{25}$, Eomes ${ }^{19}$, $\mathrm{zVegT}^{19}, \mathrm{Tbxt}^{19}$ and Tbx6) and signal mediators ( $\beta$-catenin, Smad1, Smad2) from the 1,024-cell to the early tailbud stage. pCRM were hierarchically clustered according to the binding levels of all TFs and signal mediators. The pCRM coordinates were generated by collating the strongest 2,000 peaks of each binding profile. Heat map to the right shows the occurrence of specific DNA motifs $\pm 50 \mathrm{bp}$ from the pCRM centre as well as the flanking $50 \mathrm{bp}$. The consensus sequences of these DNA motifs are shown in Supplementary Fig. 5b. Abbreviations used for the developmental timeline: 1K, 1,024-cell stage; MBT, mid-blastula transition; eG and $1 \mathrm{G}$, early and late gastrula stage; eTailbud, early tailbud stage. 
a

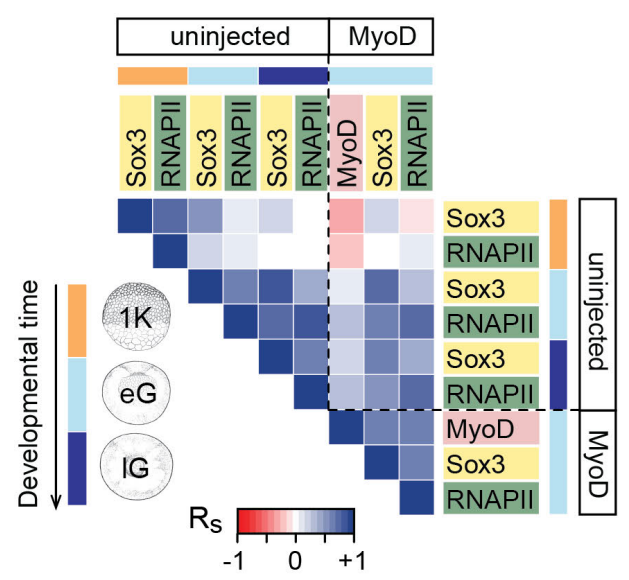

b

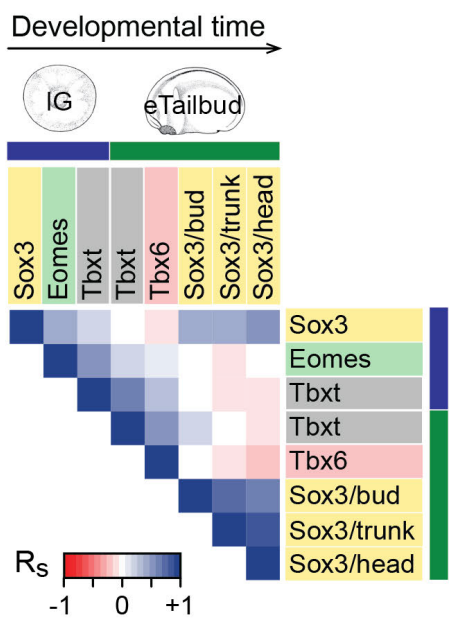

C

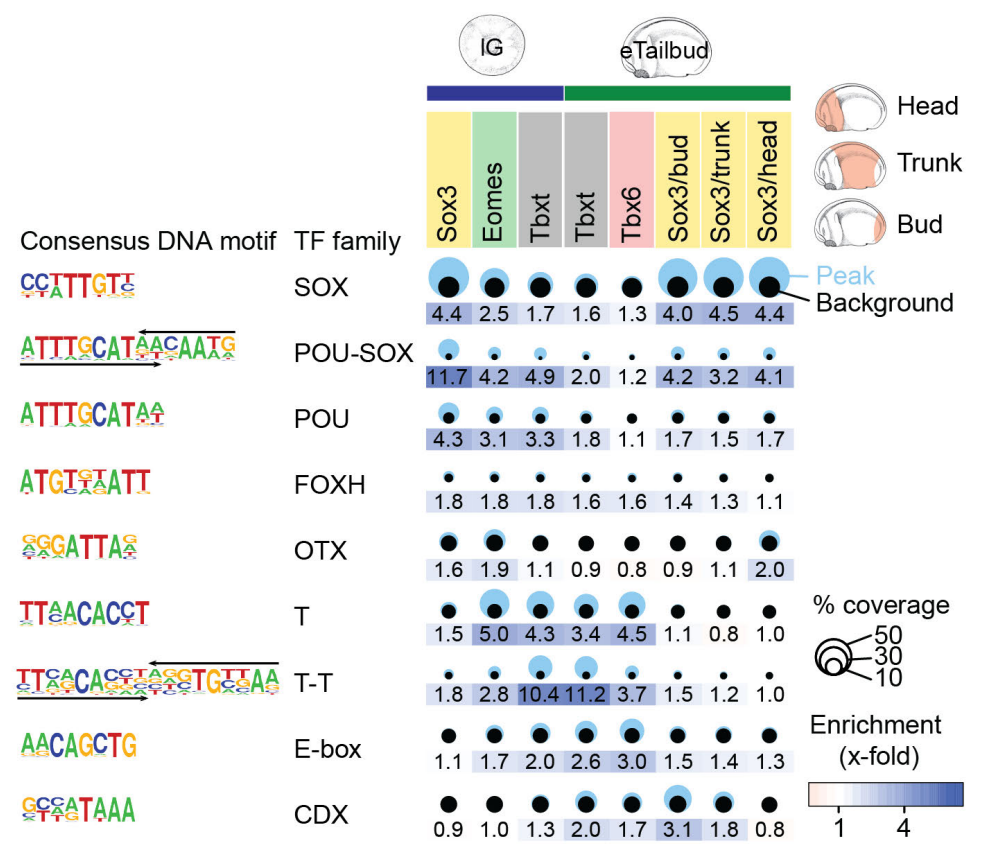

d

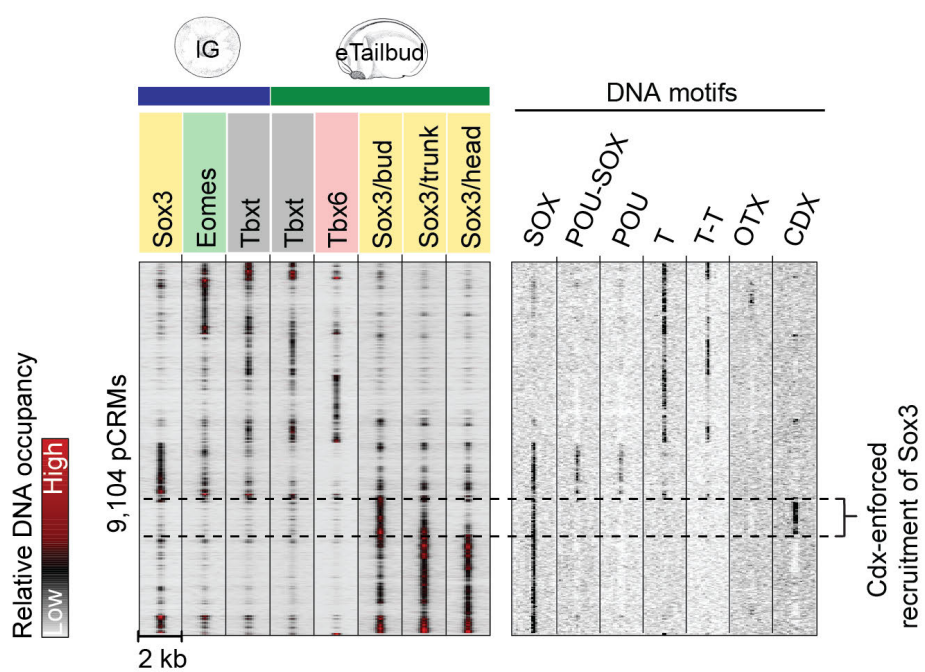

Supplementary Figure 7 TF co-expression influences chromatin engagement. (a,b) Heat maps show Spearman correlations

$580 \quad\left(\mathrm{R}_{\mathrm{s}}\right)$ of DNA occupancy levels of indicated factors in different developmental contexts. (c) Bubble plot and heat map show the 
bioRxiv preprint doi: https://doi.org/10.1101/306803; this version posted June 3, 2019. The copyright holder for this preprint (which was not certified by peer review) is the author/funder, who has granted bioRxiv a license to display the preprint in perpetuity. It is made available under aCC-BY 4.0 International license.

581 respective coverage and enrichment of DNA motifs in pCRMs bound by Sox3, Eomes ${ }^{19}$, bbxt $^{19}$ and Tbx6 in late gastrula and 582 early tailbud embryos. For Sox3 profiling, the embryo was dissected into three parts: head, trunk and tailbud. (d) Heat map 583 shows the hierarchical clustering of 9,104 pCRMs according to relative DNA occupancy levels. The pCRM coordinates were 584 generated by collating the strongest 2,000 peaks of each binding profile. The pCRM coordinates were generated by collating 585 the strongest 2,000 peaks of each binding profile. Abbreviations of the developmental timeline: 1K, 1,024-cell (early blastula); 586 eG, early gastrula; $1 \mathrm{G}$, late gastrula; and eTailbud, early tailbud. 
bioRxiv preprint doi: https://doi.org/10.1101/306803; this version posted June 3, 2019. The copyright holder for this preprint (which was not certified by peer review) is the author/funder, who has granted bioRxiv a license to display the preprint in perpetuity. It is made available under aCC-BY 4.0 International license.

587

a

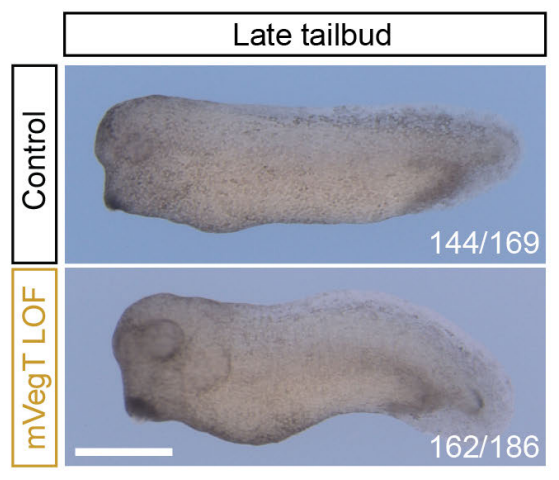

b

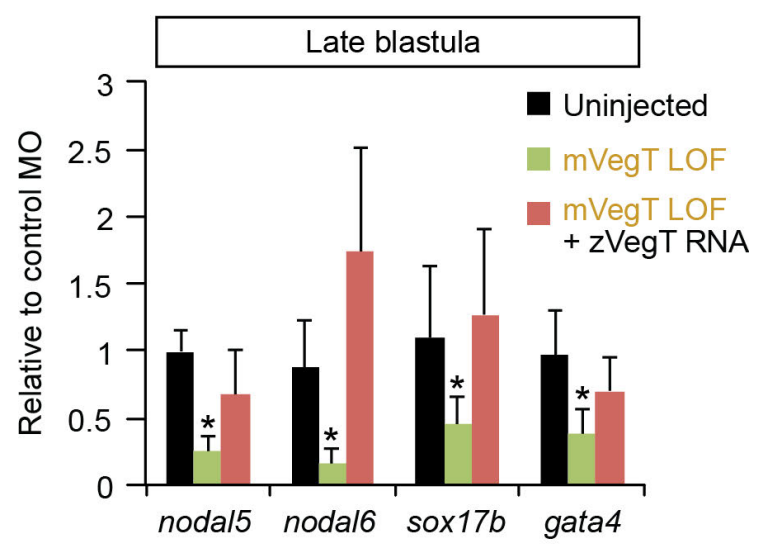

d

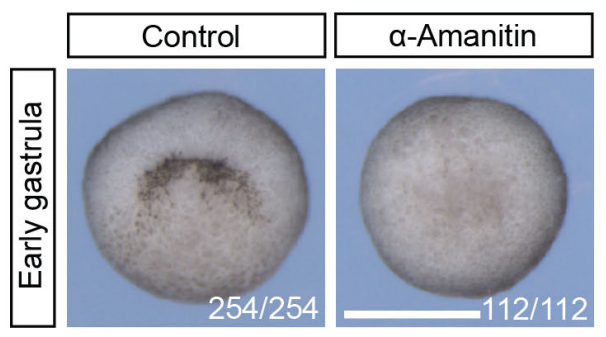

C

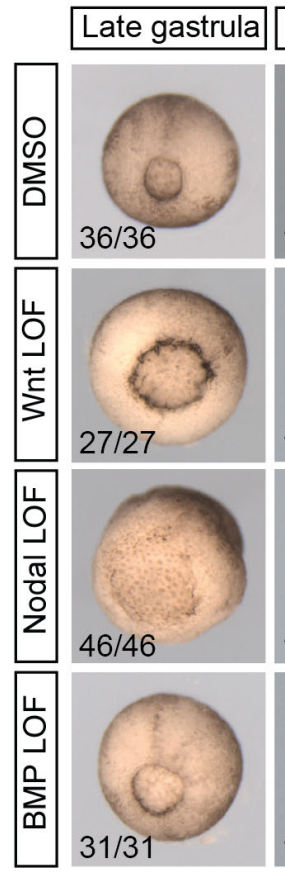

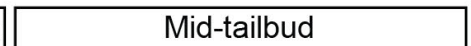
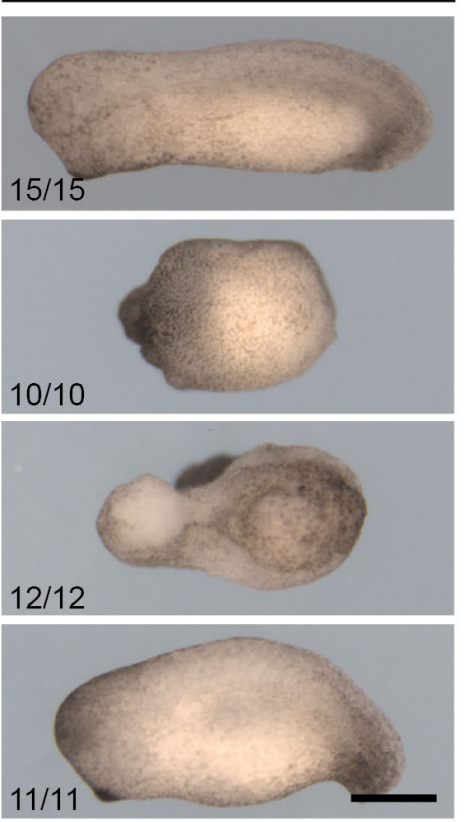

Supplementary Figure 8 Morphological defects caused by the functional loss of maternal TFs and signal mediators (a) Morphological phenotype caused by the LOF of mVegT at the late tailbud stage. (b) Bar graph shows the RT-qPCR results of rescuing reduced nodal5, nodal6, sox $17 b$ and gata4 transcript levels in mVegT LOF embryos by the injection of $X$. tropicalis zVegT mRNA. Error bars, mean+SD (n=5). Two-tailed Student's t-test: *, p <0.02. (c) Morphological phenotypes caused by the single LOF of canonical Wnt, Nodal or BMP signaling when control embryos reached the late gastrula and mid-tailbud stage. (d) Morphological phenotype caused by the injection of $\alpha$-amanitin when control embryos reached the early gastrula stage. Numbers in the right or left bottom corner of each image refer to the count of embryos detected with the displayed morphological phenotype. Scale bars, $0.5 \mathrm{~mm}$. 
597
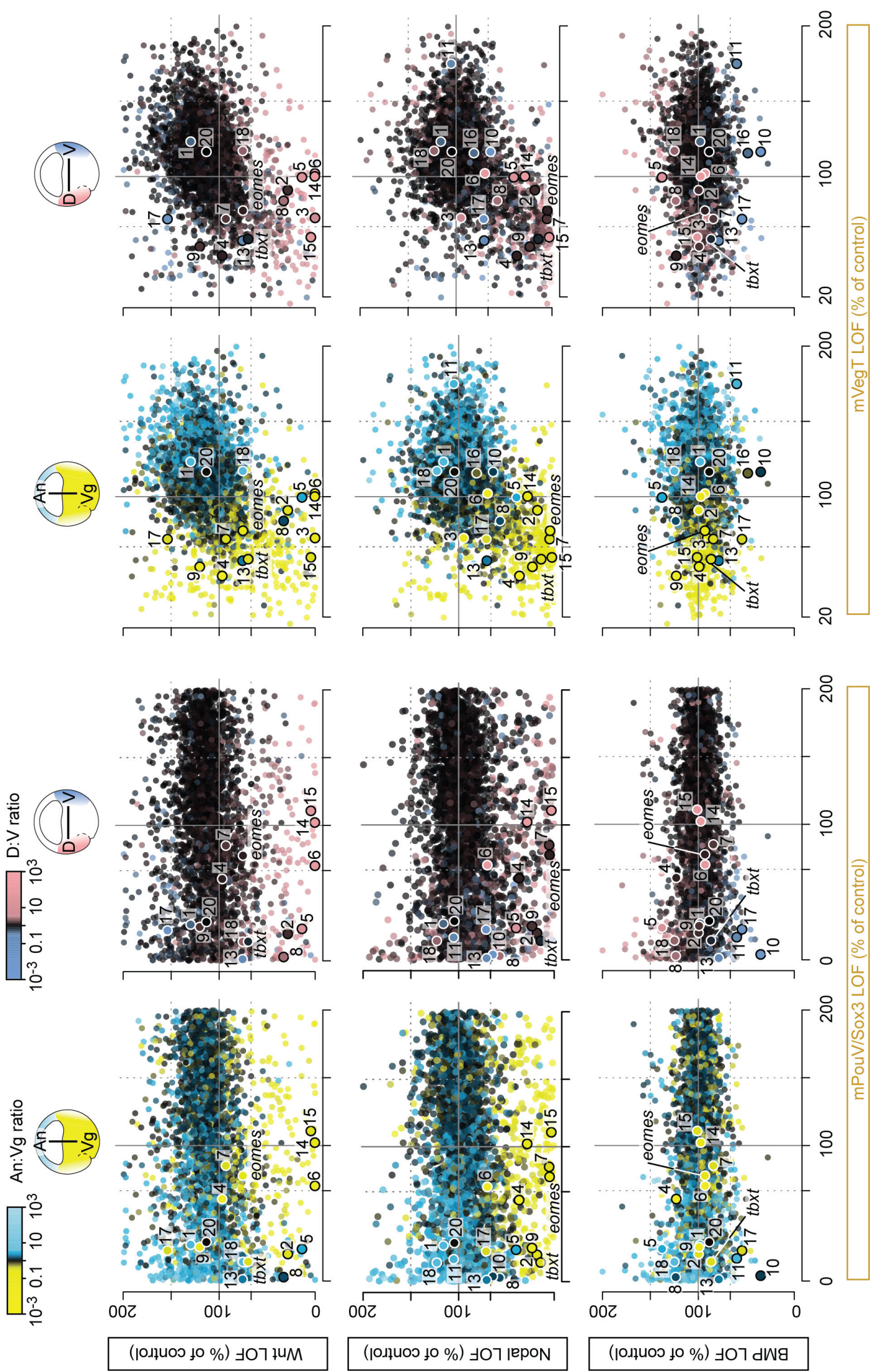

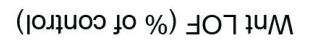

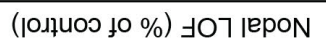

599 Supplementary Figure 9 Transcriptional comparison of zygotic genes between indicated LOFs of maternal TFs or signals.

600 Dots are colored according to the normal ratio of transcript levels (regional expression) across the animal-vegetal (An: $\mathrm{Vg}$ ) or

601 dorso-ventral (D:V) axis ${ }^{51}$. Numbered dots refer to genes listed in Fig. 6e. 
a

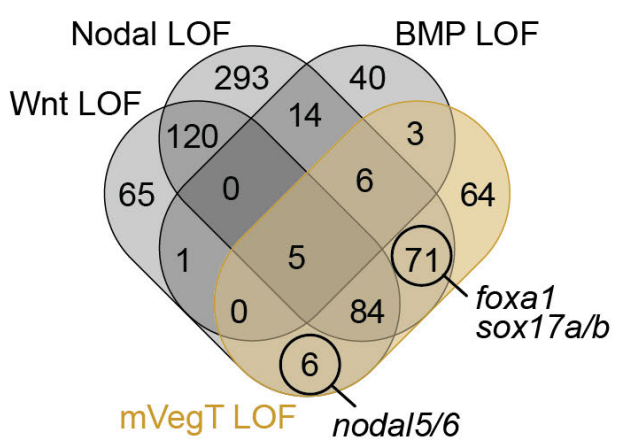

b

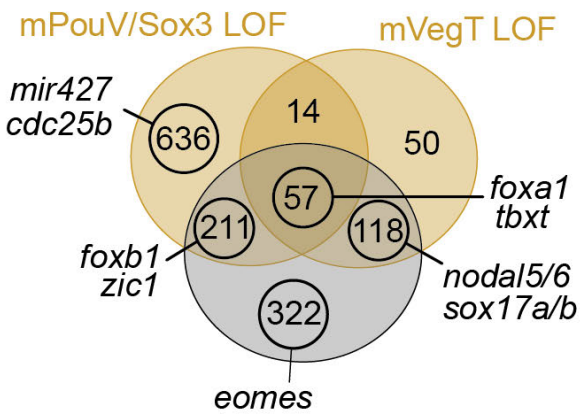

Wnt, Nodal or Bmp LOF
C
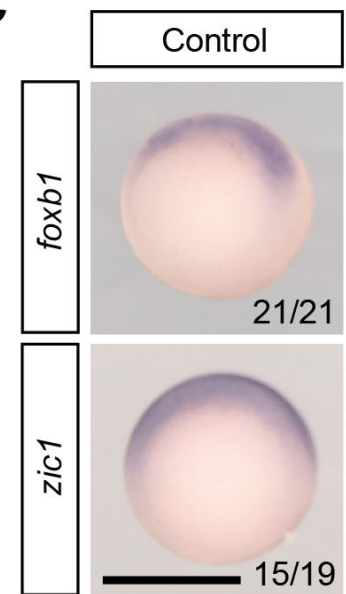

$15 / 19$
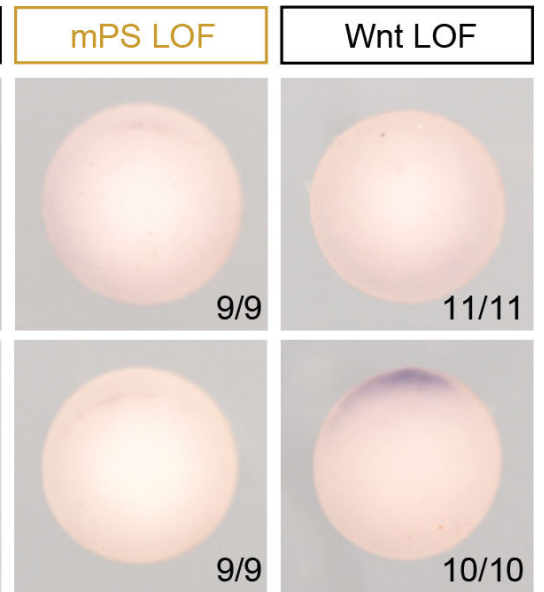

$11 / 11$

$10 / 10$ d
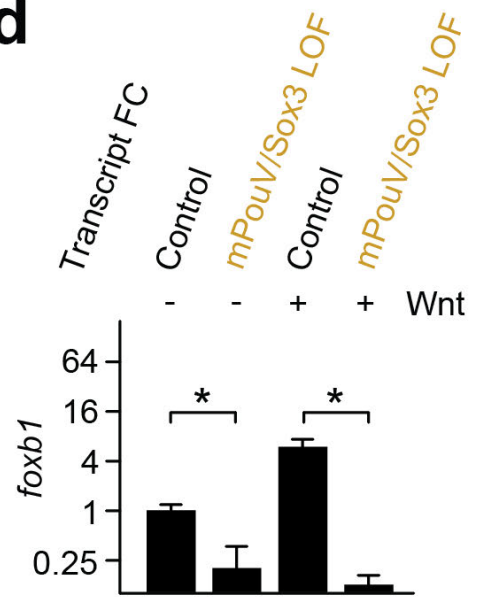

604 Supplementary Figure 10 Signal-induced regionalization of ZGA depends on maternal TFs. (a,b) Venn diagrams show the number of genes downregulated by indicated LOFs. (c) Early gastrula-staged WMISHs show that Wnt-induced transcription of foxbl and zicl on the dorsal side of the embryo depends on ubiquitously expressed maternal pluripotency factors $\mathrm{mPouV}$ and Sox3 (mPS). Embryos are imaged from the vegetal side and orientated so that dorsal side faces top. Numbers in the right bottom corner of each image refer to the count of embryos detected with the displayed WMISH staining among all embryos analyzed per condition and in situ probe. Scale bars, $0.5 \mathrm{~mm}$. (d) Bar graph shows the relative quantification of foxbl transcript levels (RT-qPCR) in control and mPouV/Sox3 LOF animal caps with or without canonical Wnt signaling. Error bars, mean $+\mathrm{SD}(\mathrm{n}=2)$. Two-tailed Student's t-test: *, $\mathrm{p}=0.05$. 
bioRxiv preprint doi: https://doi.org/10.1101/306803; this version posted June 3, 2019. The copyright holder for this preprint (which was not certified by peer review) is the author/funder, who has granted bioRxiv a license to display the preprint in perpetuity. It is made available under aCC-BY 4.0 International license.

612

a

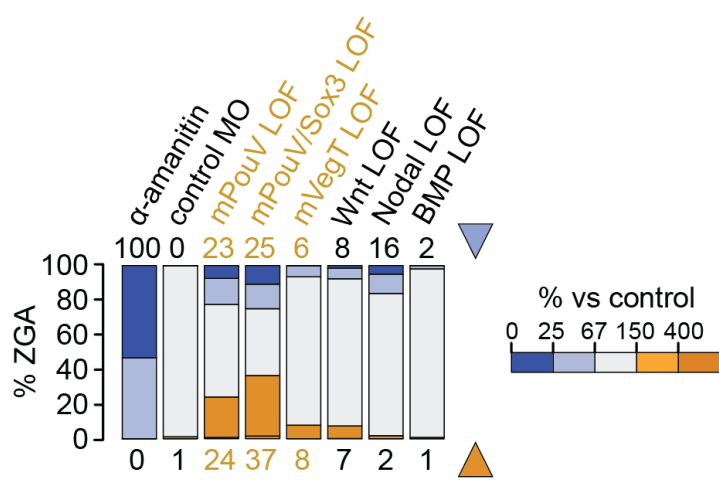

b

Regulation of Nodal signaling pathway (GO:1900107)

Spemann organizer formation (GO:0060061)

Animal organ development (GO:0048513)

Cell migration (GO:0016477)

Cell-substrate adhesion (GO:0031589)

Gastrulation (GO:0007369)

Neural tube closure (GO:0001843)

Formation of primary germ layer (GO:0001704)

Ectoderm development (GO:0007398)

Mesoderm development (GO:0007498)

Endoderm development (GO:0007492)

Pattern specification process (GO:0007389)

Dorsal/ventral pattern formation (GO:0009953)

Anterior/posterior pattern specification (GO:0009952)

Regionalization (GO:0003002)

Central nervous system development (GO:0007417)

Cardiovascular system development (GO:0072358)

Somite development (GO:0061053)

Chromosome organization (GO:0051276)

Gamete generation (GO:0007276)

DNA methylation (GO:0006306)

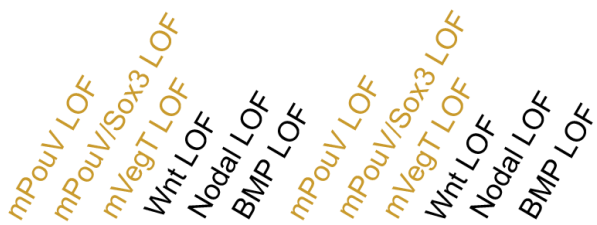

Significance ( $p$-value)

Downregulated genes Upregulated genes

\begin{tabular}{cc|c|c|c|}
$10^{-20}$ & $2-10$ & $11-30$ & $31-100$ & $101-500$ \\
$10^{-5}$ & &
\end{tabular}

614 Supplementary Figure 11 Selected maternal TFs and inductive signals regulate the regionalization of zygotic genome activation (ZGA) and the formation of primary body axes and germ layers. (a) Stacked bar graph shows the percentage of zygotic genes misregulated (\% ZGA) by indicated LOFs. (b) Bubble plot shows the enrichment of key GO term-related biological processes among the zygotic genes misregulated by indicated LOFs. Size of bubble reflects the statistical significance (hypergeometric p-value) of enrichment while the color indicates the number of affected genes. 
bioRxiv preprint doi: https://doi.org/10.1101/306803; this version posted June 3, 2019. The copyright holder for this preprint (which was not certified by peer review) is the author/funder, who has granted bioRxiv a license to display the preprint in perpetuity. It is made available under aCC-BY 4.0 International license.

\section{a}

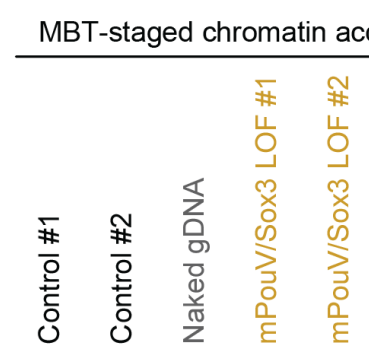

$\begin{array}{llllll}1 & 0.87 & 0.5 & 0.57 & 0.56 & \text { Control \#1 }\end{array}$

$1 \quad 0.56 \quad 0.62 \quad 0.67 \quad$ Control \#2

$1 \quad 0.650 .68$ Naked gDNA

619

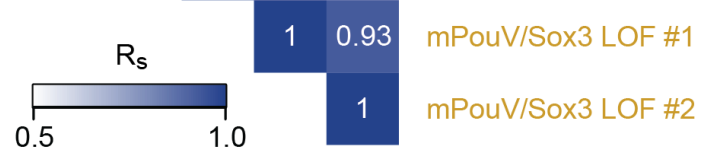

b

MBT-staged chromatin conformation capture (next-generation capture-C)

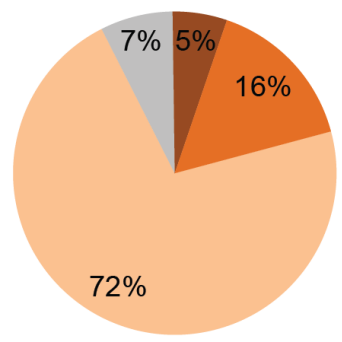

Capture+/reporter-

Capture ${ }^{-}$(contamination)

Capture ${ }^{+} /$reporter $^{+}$

Capture $^{+/ \text {reporter }^{+}}$ (redundant)

\section{$1 \mathrm{mPouV} /$ Sox3 LOF \#2}

Supplementary Figure 12 Performance of DNase-Seq and next-generation capture-C. (a) Heat map shows pairwise Spearman correlations $\left(\mathrm{R}_{\mathrm{s}}\right)$ among indicated biological replicates of MBT-staged DNase-Seq on extracted chromatin and naked genomic DNA (gDNA). (b) Pie chart summarizes the sequence composition (capture/reporter) of FLASH ${ }^{53}$ reads. Promoter contacts (capture) with distal genomic elements (reporter) were enriched by two rounds of hybridization with promoter-specific probes (see Fig. 9b and Supplementary Table 10). 
bioRxiv preprint doi: https://doi.org/10.1101/306803; this version posted June 3, 2019. The copyright holder for this preprint (which was not certified by peer review) is the author/funder, who has granted bioRxiv a license to display the preprint in perpetuity. It is made available under aCC-BY 4.0 International license.

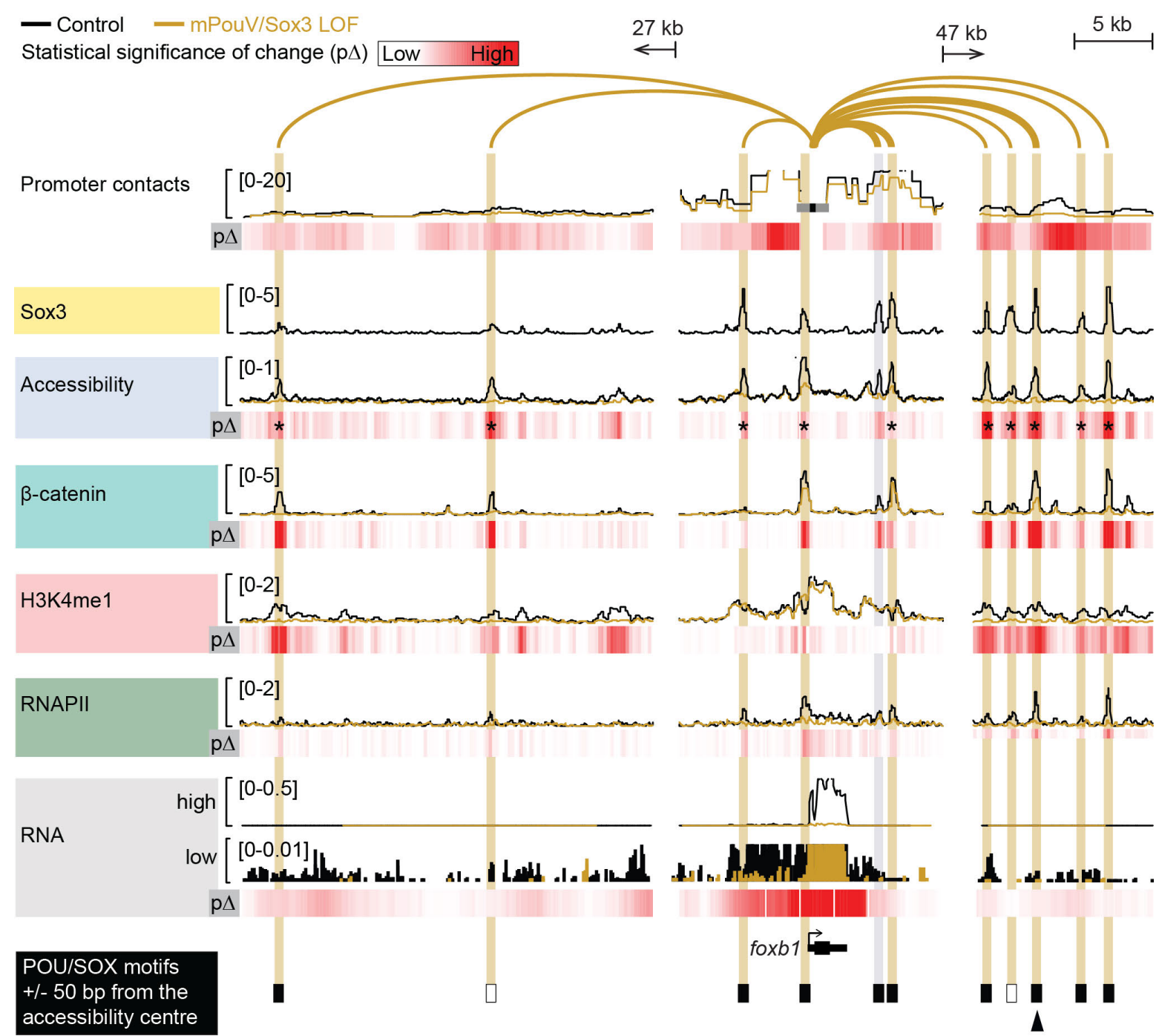
uperimposed line tracks show the level of promoter-tied chromatin conformations, chromatin accessibility and various chromatin components ( $\beta$-catenin, H3K4me1 and RNAPII) at the foxbl gene locus between control (uninjected) and $\mathrm{mPouV} / \mathrm{Sox} 3$ LOF embryos. The RNA track is split into a high (0-0.5) and low (0-0.01) expression window. Note that the low-expression window shows that locally transcribed non-coding super-enhancer RNA depend on mPouV/Sox3 as well as the gene foxb1. Heat maps $(\mathrm{p} \Delta)$ below each superimposed line plot show the statistical significance of changes caused by $\mathrm{mPouV} / \mathrm{Sox} 3 \mathrm{LOF}$. The footer highlights the occurrences of canonical POU/SOX motifs (black filled rectangles) at accessible pCRMs ( \pm 50 bp from the accessibility centre) and one strongly affected pCRM with an arrowhead. Asterisks on the p $\Delta$ heat map mark significant (FDR $\leq 10 \%$ ) reductions to pCRM accessibility. pCRMs are boxed in and their frequency of contacts with the foxbl promoter are illustrated with an arc of varying strength. Boxes of affected pCRM and arcs of promoter contacts are colored orange. 
bioRxiv preprint doi: https://doi.org/10.1101/306803; this version posted June 3, 2019. The copyright holder for this preprint (which was not certified by peer review) is the author/funder, who has granted bioRxiv a license to display the preprint in perpetuity. It is made available under aCC-BY 4.0 International license.

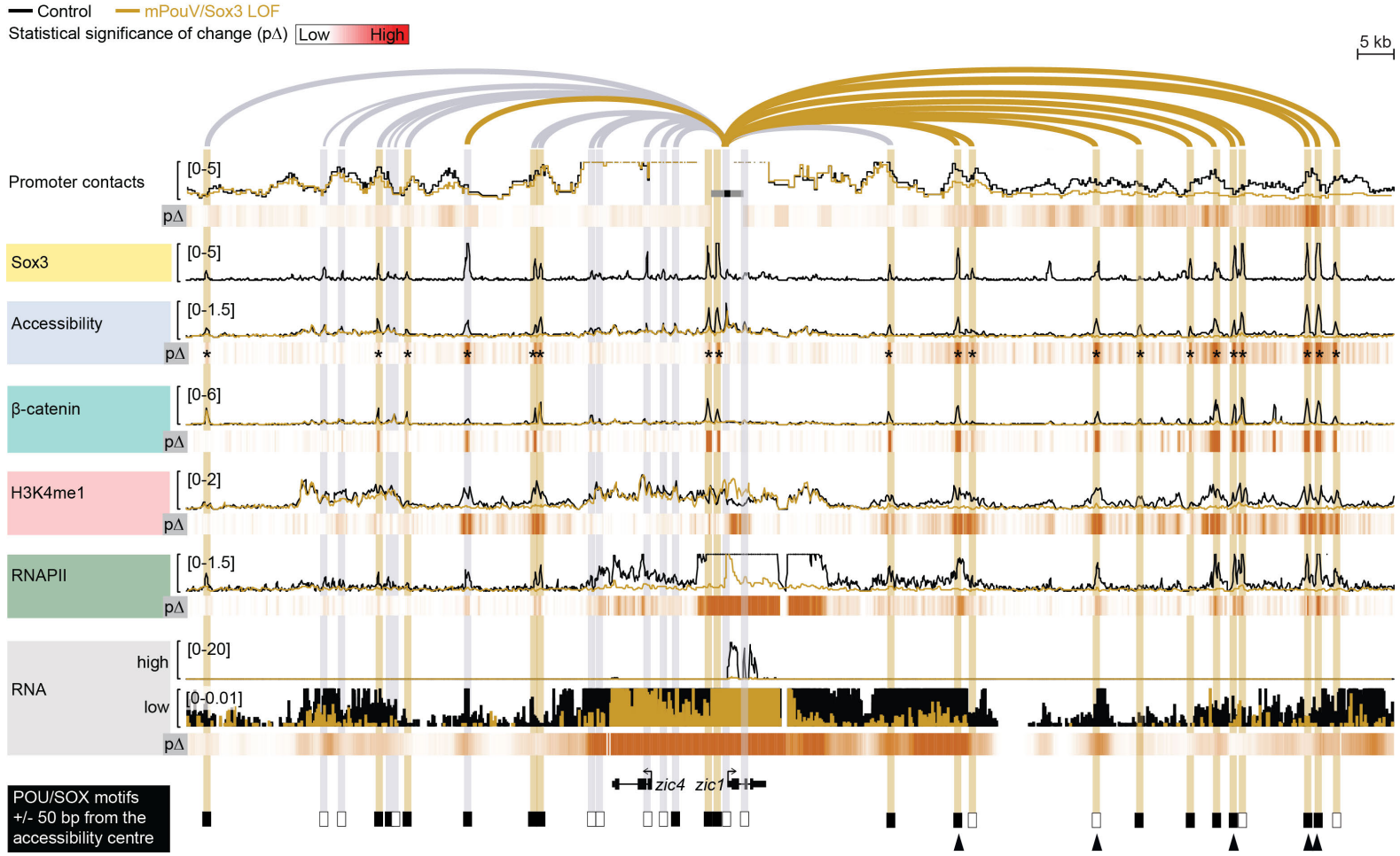

638 Supplementary Figure 14 Pioneering activity of maternal PouV/Sox3 initiates extensive chromatin remodeling.

639 Superimposed line tracks show the level of promoter-tied chromatin conformations, chromatin accessibility and various

640 chromatin components ( $\beta$-catenin, H3K4me1 and RNAPII) at the zicl gene locus between control (uninjected) and $\mathrm{mPouV} / \mathrm{Sox} 3 \mathrm{LOF}$ embryos. The RNA track is split into a high (0-20) and low (0-0.01) expression window. Note that the lowexpression window shows that locally transcribed non-coding super-enhancer RNA depend on mPouV/Sox 3 as well as the gene zicl. Heat maps $(\mathrm{p} \Delta)$ below each superimposed line plot show the statistical significance of changes caused by $\mathrm{mPouV} / \mathrm{Sox} 3 \mathrm{LOF}$. The footer highlights the occurrences of canonical POU/SOX motifs (black filled rectangles) at accessible pCRMs ( $\pm 50 \mathrm{bp}$ from the accessibility centre) and strongly affected pCRMs with arrowheads. Asterisks on the $\mathrm{p} \Delta$ heat map mark significant (FDR $\leq 10 \%$ ) reductions to $\mathrm{pCRM}$ accessibility. pCRMs are boxed in and their frequency of contacts with the $z i c 1$ promoter are illustrated with an arc of varying strength. Boxes of affected pCRMs and arcs of promoter contacts are colored orange. 
bioRxiv preprint doi: https://doi.org/10.1101/306803; this version posted June 3, 2019. The copyright holder for this preprint (which was

not certified by peer review) is the author/funder, who has granted bioRxiv a license to display the preprint in perpetuity. It is made available under aCC-BY 4.0 International license.
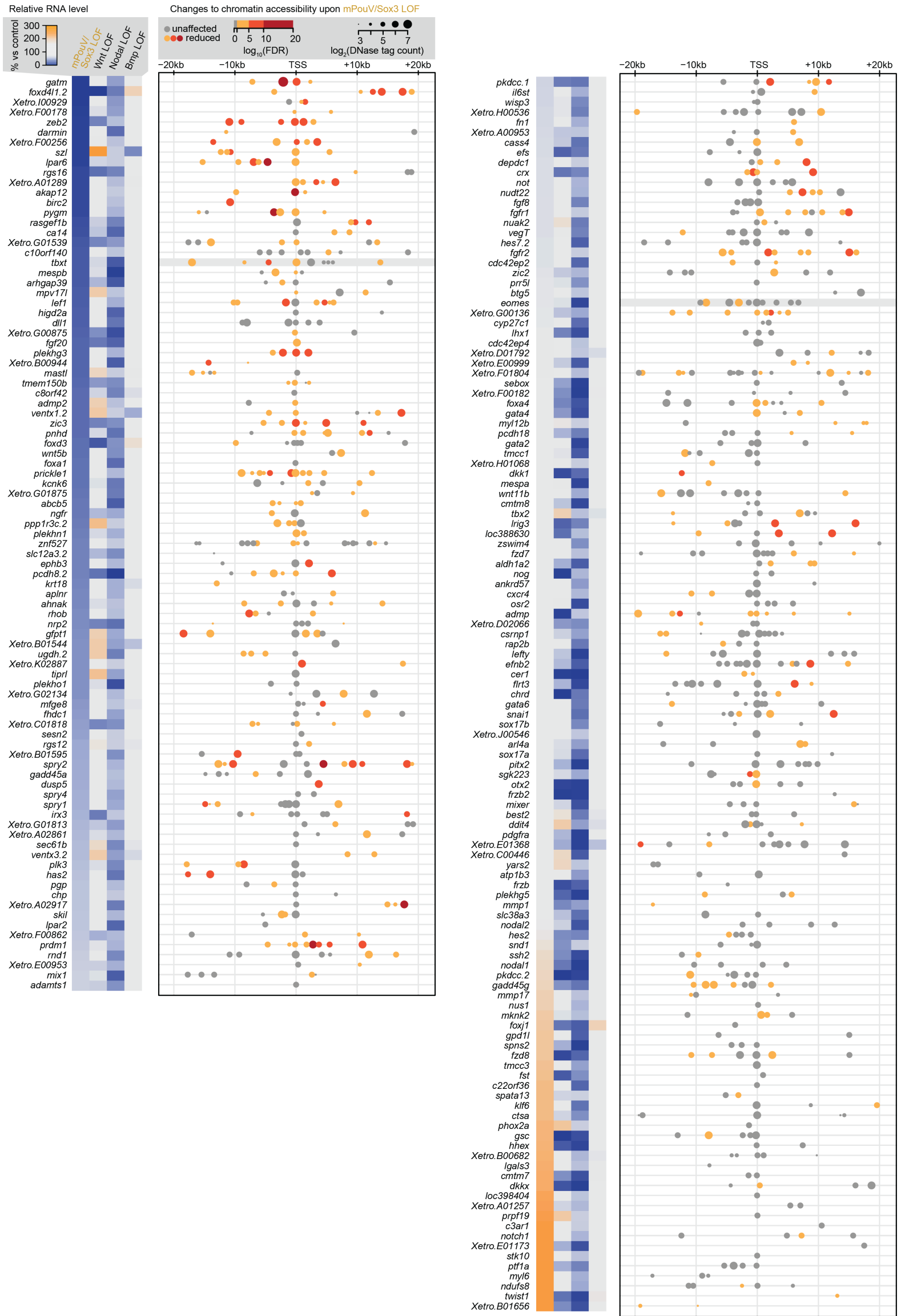

Supplementary Figure $15 \mathrm{mPouV} / \mathrm{Sox} 3$-induced chromatin accessibility is required for the expression of Nodal-responsive 
bioRxiv preprint doi: https://doi.org/10.1101/306803; this version posted June 3, 2019. The copyright holder for this preprint (which was not certified by peer review) is the author/funder, who has granted bioRxiv a license to display the preprint in perpetuity. It is made available under aCC-BY 4.0 International license.

652 active by the $\mathrm{MBT}^{52}$ and their transcript levels are significantly reduced ( $\geq$ two-fold; FDR $\leq 10 \%$ ) upon $\alpha$-amanitin injection (see Fig. 6e). The plot aligned to the heat map shows the localization and DNase sensitivity (bubble size) of accessible pCRMs (affected, dot colored in orange to red with FDR decreasing from 10\%; and unaffected, grey dot) relative to zygotic TSSs. Gene loci are sorted by mPouV/Sox 3 LOF-induced transcript fold changes as shown in the heat map. 
bioRxiv preprint doi: https://doi.org/10.1101/306803; this version posted June 3, 2019. The copyright holder for this preprint (which was

not certified by peer review) is the author/funder, who has granted bioRxiv a license to display the preprint in perpetuity. It is made available under aCC-BY 4.0 International license.

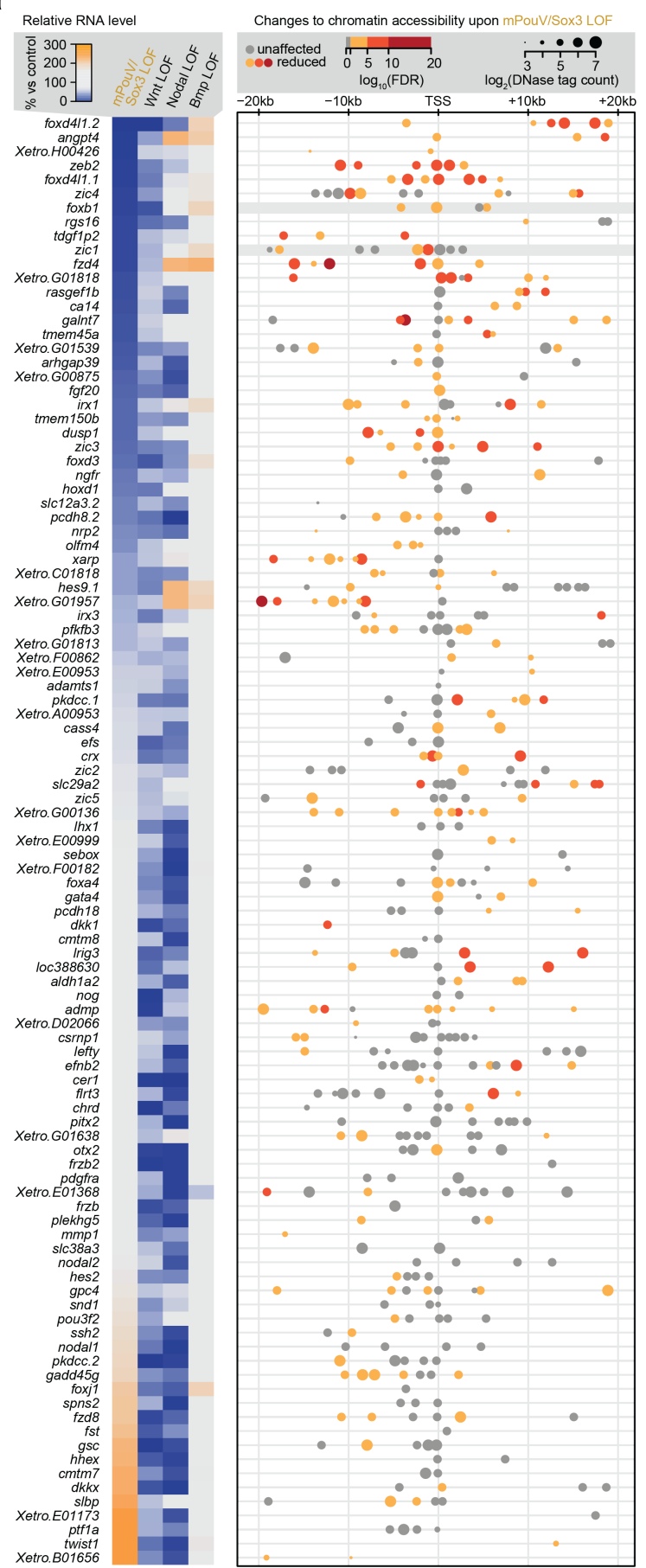

b

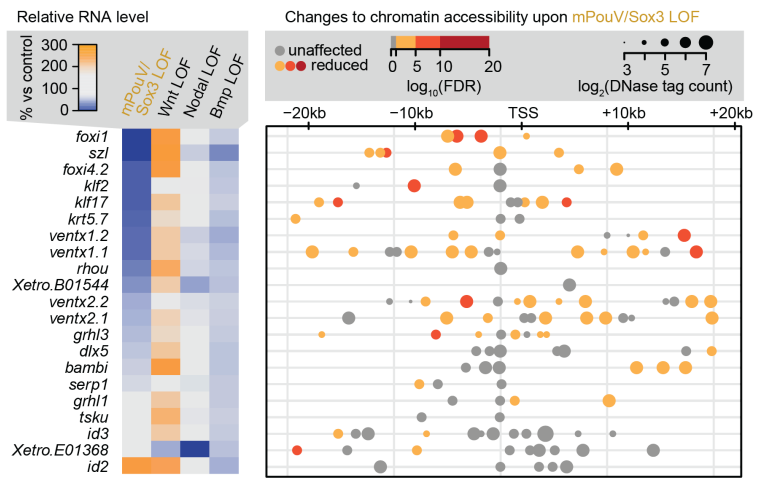

Supplementary Figure $16 \mathrm{mPouV} / \mathrm{Sox} 3$-induced chromatin accessibility is required for the expression of Wnt- and BMP responsive genes. $(\mathbf{a}, \mathbf{b})$ Heat maps show the transcript levels of Wnt- or Bmp-responsive genes under indicated LOFs. All genes listed here are active by the $\mathrm{MBT}^{52}$ and their transcript levels are significantly reduced ( $\geq$ two-fold; FDR $\leq 10 \%$ ) upon $\alpha$-amanitin injection (see Fig. 6e). The plot aligned to the heat map shows the localization and DNase sensitivity (bubble size) of accessible pCRMs (affected, dot colored in orange to red with FDR decreasing from 10\%; and unaffected, grey dot) relative to zygotic TSSs. Gene loci are sorted by mPouV/Sox3 LOF-induced transcript fold changes as shown in the heat map. 
bioRxiv preprint doi: https://doi.org/10.1101/306803; this version posted June 3, 2019. The copyright holder for this preprint (which was

not certified by peer review) is the author/funder, who has granted bioRxiv a license to display the preprint in perpetuity. It is made available under aCC-BY 4.0 International license.
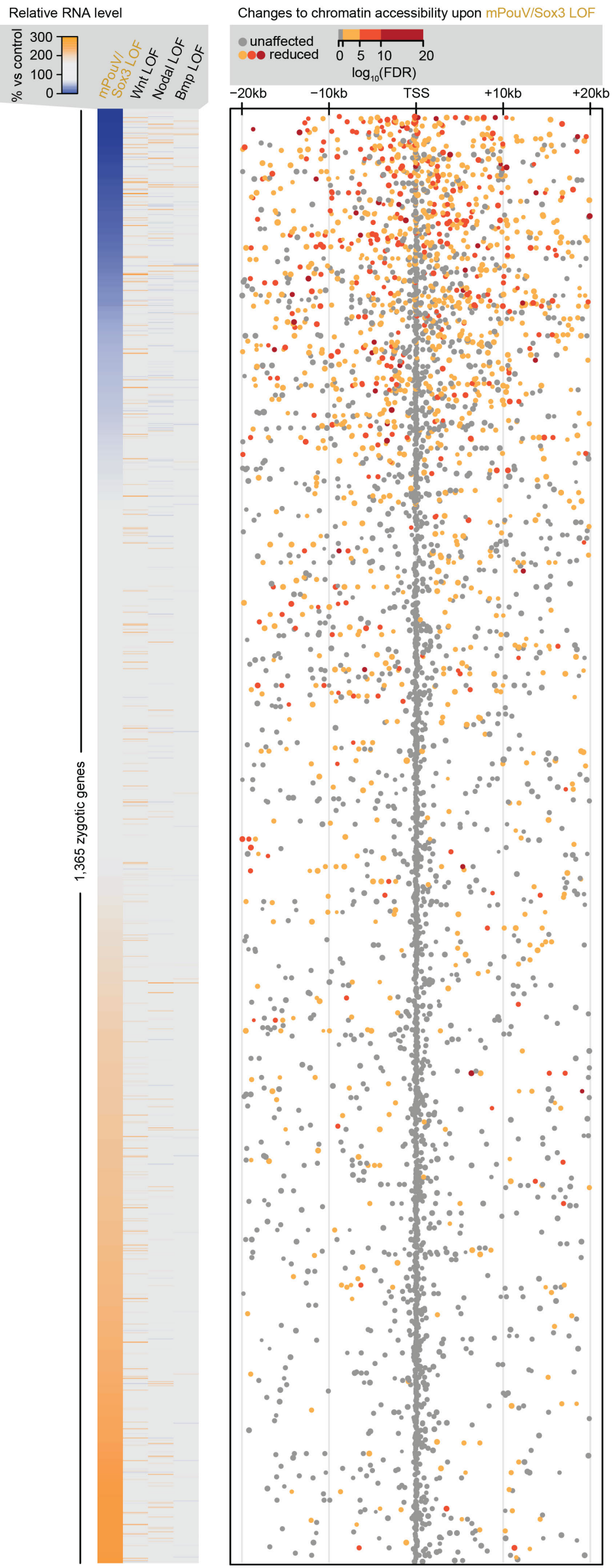
Supplementary Figure $17 \mathrm{mPouV} / \mathrm{Sox} 3$-induced chromatin accessibility is required for the expression of signal nonresponsive genes. The heat map shows the transcript levels of 1,365 signal non-responsive genes under indicated LOFs. The plot aligned to the heat map shows the localization of accessible pCRMs (affected, dot colored in orange to red with FDR decreasing from 10\%; and unaffected, grey dot) relative to the zygotic TSSs. All genes listed here are active by the MBT ${ }^{52}$ and their transcript levels are significantly reduced ( $\geq$ two-fold; FDR $\leq 10 \%$ ) upon $\alpha$-amanitin injection (see Fig. 6e). Gene loci are sorted by $\mathrm{mPouV} / \mathrm{Sox} 3 \mathrm{LOF}-$ induced transcript fold changes as shown in the heat map.

670

Supplementary Movie 1 Gastrulation defects caused by the loss-of-function of maternal pluripotency factors.

Supplementary Table 1 Summary of deep sequencing and read alignments.

Supplementary Table 2 Annotation of RNAPII ${ }^{+}$pCRMs (genomic coordinates, distance to nearest TSS, DNA occupancy levels from the 32-cell to the late gastrula stage, DNA motif counts)

676 Supplementary Table 3 Annotation of accessible pCRMs (genomic coordinates, distance to nearest TSS, normalized DNase cleavage count and DNA occupancy levels of RNAPII and H3K4me1)

678 Supplementary Table 4 Maternal protein concentrations versus ribosome densities (translation) across the mid-blastula 679 transition.

680 Supplementary Table 5 ChIP-Seq peak calling coordinates for TFs (Eomes, Foxh1, Sox3, Tbx6, Tbxt and VegT) and signal mediators (catenin, Smad1 and Smad2) at indicated developmental stages.

682 Supplementary Table 6 Genomic coordinates of super-enhancers assembled from individual TF and signal mediator ChIP-Seq profiles separated by developmental stages (up to MBT, early gastrula stage and late gastrula stage)

684 Supplementary Table 7 ChIP-Seq peak calling coordinates for Sox3 and MyoD-HA in early gastrula embryos with ectopic expression of MyoD-HA.

Supplementary Table 8 ChIP-Seq peak calling coordinates for Sox3 in dissected parts of the early tailbud embryo (head, trunk and bud).

688 Supplementary Table 9 Transcriptional effect of maternal transcription factors and inductive signals on zygotic genome 689 activation.

690 Supplementary Table 10 Genomic coordinates of selected capture-C viewpoints and DNA sequences used for capturing promoter-tied regulatory elements.

692 Supplementary Table 11 MBT-staged chromatin accessibility changes caused by mPouV/Sox3 LOF.

693 Supplementary Table 12 Results of the next-generation capture-C performed at the MBT on control and $\mathrm{mPouV} / \mathrm{Sox} 3 \mathrm{LOF}$ 694 embryos.

697 Xenopus manipulation. Standard procedures were used for ovulation, fertilization, and manipulation and incubation of embryos $^{55,56}$. Briefly, frogs were obtained from Nasco (Wisconsin, USA). Ovulation was induced by injecting serum gonadotropin (Intervet) and chorionic gonadotropin (Intervet) into the dorsal lymph sac of mature female frogs. Eggs were fertilized in vitro with sperm solution consisting of 90\% Leibovitz's L-15 medium (Gibco) and 10\% fetal calf serum (Gibco). After 10 min, fertilized eggs were de-jellied with 2.2\% (w/v) L-cysteine (Sigma) equilibrated to $\mathrm{pH}$ 8.0. X. tropicalis embryos were cultured in 5\% Marc's 
at $14^{\circ} \mathrm{C}-22^{\circ} \mathrm{C}$. Embryos were staged as previously described ${ }^{57}$. All Xenopus work fully complied with the UK Animals (Scientific Procedures) Act 1986 as implemented by the Francis Crick Institute.

Nucleic acid injections and treatments of embryos. Microinjections were performed using calibrated needles and embryos equilibrated in 4\% (w/v) Ficoll PM-400 (Sigma) in 5\% MMR or 10\% NAM. Microinjection needles were generated from borosilicate glass capillaries (Harvard Apparatus, GC120-15) using the micropipette puller Sutter p97. Maximally two nanolitres of morpholino (MO) and/or mRNA were injected into de-jellied embryos at the 1-cell, 2-cell or 4-cell stage using the microinjector Narishige IM-300. Injections for the mVegT loss-of-function (LOF) and rescue were targeted to the vegetal hemisphere. All other injections were targeted to the animal hemisphere. Embryos were transferred to fresh 5\% MMR or 10\% NAM (without Ficoll) once they reached about blastula stage.

The following mRNA amounts were used for ectopic protein expression: $80 \mathrm{pg}$ and $400 \mathrm{pg}$ MyoD-HA mRNA into X. tropicalis and $X$. laevis embryos, respectively; and $400 \mathrm{pg}$ Sox3-HA into $X$. laevis embryos.

The following MO amounts and treatments were used for LOF experiments: $5 \mathrm{ng}$ Pou5f3.2 MO, $5 \mathrm{ng}$ Pou5f3.3 MO and $5 \mathrm{ng}$ standard control MO (mPouV LOF); $5 \mathrm{ng}$ Pouff3.2 MO, $5 \mathrm{ng}$ Pouff3.3 MO and 5 ng Sox3 MO (mPouV/Sox3 LOF); 5 ng $\beta$ catenin MO (Wnt LOF); $10 \mathrm{ng} \mathrm{mVegT} \mathrm{MO} \mathrm{(mVegT} \mathrm{LOF);} \mathrm{standard} \mathrm{control} \mathrm{MO} \mathrm{(5-10} \mathrm{ng} \mathrm{according} \mathrm{to} \mathrm{the} \mathrm{dose} \mathrm{used} \mathrm{for} \mathrm{the} \beta$ catenin or mVegT LOF experiment); and $30 \mathrm{pg} \alpha$-amanitin (BioChemica, \#A14850001). To block Nodal (Nodal LOF) or BMP (BMP LOF) signaling, embryos were treated with $100 \mu \mathrm{M}$ SB431542 (Tocris, \#1614) or $10 \mu \mathrm{M}$ LDN193189 (Selleckchem, \#S2618) from the 8-cell stage onwards. Control embryos were treated accordingly with DMSO, in which these antagonists were dissolved. MOs were designed and produced by Genetools (Oregon, USA) to block translation. $\beta$-catenin MO (5'TTTCAACAGTTTCCAAAGAACCAGG-3', underlined base was changed from the original $\mathrm{MO}^{26}$ ), Pou5f3.2 MO (5'GCTGTTGGCTGTACATAGTGTC-3', underlined base was changed from the original $\mathrm{MO}^{25}$ ), Pou5f3.3 $\mathrm{MO}^{25}$ (5'GTACAGAACGGgtTGGTCCATGTTC-3'), Sox3 MO (5'-GTCTGTGTCCAACATGCTATACATC-3'), Tbx6 MO (5'TACATTGGGTGCAGGGACCCTCTCA-3') and mVegT MO $^{58}$ (5'-TGTGTTCCTGACAGCAGTTTCTCAT-3'; see Supplementary Fig. 2e).

For the rescue of mVegT LOF, 25-50 pg zVegT mRNA were injected at the 2- or 4-cell stage. For the rescue of mPouV/Sox3 LOF, 75 pg Pouff3.3 mRNA and 75 pg Sox 2 mRNA were injected at the 2- or 4-cell stage. For both rescue experiment, 150-300 pg $m$ Cherry mRNA was co-injected to trace mRNA expression in embryonic cells.

RNA constructs. All constructs are products of Gateway (Invitrogen) cloning. First, the coding sequences of interest were amplified from either plasmid using PfuTurbo DNA polymerase (Agilent, \#600250) or cDNA using Phusion HF DNA polymerase (NEB, \#M0530), and unidirectionally cloned into the pENTR/D-TOPO vector (Thermo Fisher Scientific, \#K2400). Next, these clones were LR-recombined with Gateway-compatible pCS2+ vectors containing no tag or a C-terminal 3xHA tag (see Fig. 4g). The following forward and reverse primers were used for PCR amplification: X. laevis myoD1a from MyoD2-24 pBluescript plasmid $^{59}$, 5'-CACCATGGAGCTGTTGCCCCCAC-3', 5'-TAAGACGTGATAGATGGTGCTG-3'; X. laevis sox3 from the IMAGE:443920 clone (European Xenopus Resource Centre), 5'-CACCATGTATAGCATGTTGGACAC-3', 5'TATGTGAGTGAGCGGTACAGTG-3'; X. laevis sox2 from stage 11 cDNA, 5'-CACCATGTACAGCATGATGGAGACCGA3', 5'-TCACATGTGCGACAGAGGCAGC-3'; $X$. laevis pou5f3.3 from stage 11 cDNA, 5'CACCATGGACCAGCCCATATTGTACA-3', 5'-TCAGCCGGTCAGGACCCCCA-3'; X. tropicalis zVegT from stage 11 cDNA, 5'-CACCATGCACTCTCTGCCGGATGTA-3', 5'-TTACCAACAGCTGTATGGAAAGA-3'. pCS2+8NmCherry ${ }^{60}$ (\#34936) was obtained from Addgene. 
enzymes (NEB) were used for linearizing the pCS2+ constructs: Asp781 (myoDla, sox3), PvuII (sox2), PspOMI (pou5f3.3, zVegT) and NotI (mCherry).

Generation of digoxigenin-labeled RNA probes. DNA template for generating the foxbl in situ hybridization probe was amplified from $X$. tropicalis stage 19-20 cDNA. KAPA HiFi HotStart polymerase (Kapa Biosystems, \#KK2602) and the following PCR cycling conditions were used: $98^{\circ} \mathrm{C}$ for $45 \mathrm{sec}$ followed by 40 cycles $\left(98^{\circ} \mathrm{C}\right.$ for $10 \mathrm{sec}, 63^{\circ} \mathrm{C}$ for $10 \mathrm{sec}, 72^{\circ} \mathrm{C}$ for $\left.15 \mathrm{sec}\right)$ and final elongation step of $20 \mathrm{sec}$ at $72^{\circ} \mathrm{C}$. Primers were 5'-GTCAGCGCCTATGGAGTACC-3' and 5'AACACTGGAGATGCCATGC-3'. Fresh PCR product was zero-blunt cloned into the pCRII-TOPO vector (Thermo Fisher Scientific, \#K2800). The identity and direction of insert was verified by restriction digest and Sanger sequencing. Plasmids were linearized by restriction digestion and purified using the QIAquick PCR purification kit (Qiagen, \#28104). All in situ hybridization probes were transcribed from $\sim 1 \mu \mathrm{g}$ linearized plasmid using 1x digoxigenin-11-UTP (Roche, \#11277065910), 40 U RiboLock RNase inhibitor (Thermo Fisher Scientific, \#EO0381), 1x transcription buffer (Roche) and T7 RNA polymerase (Roche, $\# 10881767001$ ) at $37^{\circ} \mathrm{C}$ for $2 \mathrm{~h}$. The probe was treated with $2 \mathrm{U}$ Turbo DNase (Thermo Fisher Scientific, \#AM2238) to remove the DNA template and purified by spin-column chromatography (Clontech). RNA was diluted to $10 \mathrm{ng} / \mu \mathrm{l}$ (10x stock) with hybridization buffer (50\% formamide, 5x SSC, 1x Denhardt's, $10 \mathrm{mM}$ EDTA, $1 \mathrm{mg} / \mathrm{ml}$ torula RNA, $100 \mu \mathrm{g} / \mathrm{ml}$ heparin, $0.1 \%$ Tween-20 and $0.1 \%$ CHAPS) and stored at $-20^{\circ} \mathrm{C}$. The following plasmids and restriction enzymes (NEB) were used for plasmid linearization: X. laevis eomes pCRII-TOPO ${ }^{19}$, BamHI; X. tropicalis foxb1 pCRII-TOPO, BamHI; X. tropicalis mir427 pCS108 (IMAGE:7545411) ${ }^{50}$, SalI; X. laevis ventx 2.1 pBluescript SK- ${ }^{61}$, EcoRI; X. laevis tbxt (Xbra) pSP73 ${ }^{62}$, BglII; and X. tropicalis zic1 pCMV-SPORT6 (IMAGE:7668846), SalI.

Animal cap assays. These assays were carried out for the ectopic expression of mRNA constructs in X. laevis and for the MOmediated LOF in $X$. tropicalis. All animal caps were dissected at the blastula stage (stage 8 to 9 ) and cultured in $75 \%$ NAM containing $0.1 \%$ bovine serum albumin (Sigma). Dissections were carried out with $13 \mu \mathrm{m}$ loop electrodes (Protech International, 13-Y1) connected to a MC-2010 micro cautery instrument (Protech International) operating at power level 2. As illustrated in Fig. 4g, control (uninjected) and MyoD-HA or Sox3-HA expressing animal caps were cultured at $20^{\circ} \mathrm{C}$ until sibling embryos reached early neurula stage (stage 13). For the experiment shown in Fig. 7d and Supplementary Fig. 10d, animal caps of control and $\mathrm{mPouV} / \mathrm{Sox} 3 \mathrm{LOF}$ embryos were cultured with or without $10 \mathrm{ng} / \mathrm{ml}$ recombinant human activin A (Nodal agonist; R\&D Systems, \#338-AC) or $50 \mu \mathrm{M}$ CHIR99021 (canonical Wnt agonist; Tocris, \#4423) at $22^{\circ} \mathrm{C}$ for $\sim 2 \mathrm{~h}$ until sibling embryos reached early gastrula stage (stage $10^{+}$).

Extraction of total RNA. 10-15 embryos (or 15-20 animal caps) were homogenized in 800 (400) $\mu 1$ TRIzol reagent (Thermo Fisher Scientific, \#15596018) by vortexing. The homogenate was either snap-frozen in liquid nitrogen and stored at $-80^{\circ} \mathrm{C}$ or processed immediately. For phase separation, the homogenate together with $0.2 \mathrm{x}$ volume of chloroform was transferred to prespun 1.5-ml Phase Lock Gel Heavy microcentrifuge tubes (VWR), shaken vigorously for $15 \mathrm{sec}$, left on the bench for 2 min and spun at $\sim 16,000 \mathrm{~g}\left(4^{\circ} \mathrm{C}\right)$ for $5 \mathrm{~min}$. The upper phase was mixed well with one volume of $95-100 \%$ ethanol and spun through the columns of Zymo RNA Clean and Concentrator 5 or 25 (Zymo Research) at 12,000 g for $30 \mathrm{sec}$. Next, the manufacturer's instructions were followed for the recovery of total RNA (>17 nt) with minor modifications. First, the flow-through of the first spin was re-applied to the column. Second, the RNA was treated in-column with 3 U Turbo DNase (Thermo Fisher Scientific, \#AM2238). Third, the RNA was eluted twice with $25 \mu 1$ molecular-grade water. The concentration was determined on the NanoDrop 1000 spectrophotometer. 
$\min , 55^{\circ} \mathrm{C}$ for $45 \mathrm{~min}, 85^{\circ} \mathrm{C}$ for $15 \mathrm{~min}$ and $4^{\circ} \mathrm{C}$ for indefinite. The reaction was diluted with $90-190 \mu 1$ molecular-grade water before using 1-2.5 $\mu 1$ for each qPCR reaction.

qPCR primers. The PCR primers were designed to hybridize at $\sim 60^{\circ} \mathrm{C}\left(\mathrm{T}_{\mathrm{m}}\right)$ and to generate $75-125 \mathrm{bp}$ amplicons. Where possible, primers were checked for their specificity in silico using Jim Kent's PCR tool (http://genomes.crick.ac.uk/cgi-bin/hgPcr). All primer pairs were tested to produce one amplicon of the correct size through diagnostic PCR using RedTaq DNA polymerase (Sigma, \#D4309). Exceptions were previously designed primer pairs for X. laevis actc1, odc1 and otx2, all of which generate amplicons of 200-250 bp.

799

Primers (forward, reverse) for X. laevis (L) or X. tropicalis (T) cDNA: actc1 (L, 5'-TCCTGTACGCTTCTGGTCGTA-3', 5'TCTAGGAGAATCCGCAGGAG-3'); foxb1 (T, 5'-CCGGATGCAGAGTGACAAC-3', 5'-GAGGCTTCTGGTCGCTGTAG3'); gata4 (T, 5'-TCCTCTGATCAAGCCTCAAAG-3', 5'-ACGGCGCCAGAGTGTAGTAG-3'); myll (L, 5'GCACCTGCATTTGATCTGTC-3', 5'-TTGCTGTCTCCTGTCCTGTC-3'); nodal5 (T, 5'-TTCAAGCCAACAAACCACGC3', 5'-CGCATCTTCACCGGTATGCA-3'); nodal6 (T, 5'-CGCATTTCGTTGTGAGGGGT-3', 5'TCGTACAGCTTGACCAAACTCT-3'); odc1 (L, 5 GCCATTGTGAAGACTCTCTCC-3', 5'TTCGGGTGATTCCTTGCCAC-3'); odcl (T, 5'-GGGCAAAAGAGCTTAATGTGG-3', 5'-CATCGTGCATCTGAGACAGC3'); otx2 (L, 5'-TTGAACCAGACCTGGACT-3', 5'-CGGGATGGATTTGTTGCA-3'); rpl8 (T, 5'CTGGCTCCAAGAAGGTCATC-3', 5'-CAGGATGGGTTTGTCAATACG-3'); sox2 (L, 5' - ACATCACATGGACGGTTGTC3', 5'-TCTCTCGGTTTCTGCTCTCG-3'); $\operatorname{sox} 17 b \quad$ (T, 5'- GTtTATGGTGTGGGCAAAGG-3', 5'810 TCAGCGATTTCCATGATTTG-3'); tbx6 (L, 5'-GGGGCACCACGACTCTTAC-3', 5'-CCCAGAATCCTCTGTGAAGG-3'); and $t b x t$ (T, 5'-CCTGTGGATGAGGTTCAAGG-3', 5'-CACGCTCACCTTTAGAACTGG-3').

812 Primers (forward, reverse) for $X$. tropicalis genomic DNA: $\beta$-actin -0.5kb (5'-TTGTACGCAACACAATGCAG-3', 5'AAATGTCAGGACCAAATGCAG-3'); $\quad d l c \quad-3.2 \mathrm{~kb} \quad$ (5'-CATCCTGCAATTAACTGTCTGC-3', 5'GTCAGGAGGGAGGTGTCAAC-3'); hoxdl $\quad+3.2 \mathrm{~kb}$ (5'-TGtTGtaGatgCTGATGCTTATCG-3', 5'GGGGAGGCCCTTTTATACAG-3');

myf5 $\quad-1.1 \mathrm{~kb}$
TCCCAAATCATCAGCACAAC-3'); AGGTAACCCCTGAGCTGTCA-3'); GGAACTCTCTGGGTCCCAAC-3');

pax3 $+2.7 \mathrm{~kb}$ AAACCCAAGGTGAGCAAGTG-3'); sial $-0.2 \mathrm{~kb}$ CAAAGGGCTTCTCTTTGTGC-3'); sox3 TSS CACGGGAGAAAATGAAGCTG-3'); TCTCCACCTAAGGGGAAACC-3'); wnt8a $-5.7 \mathrm{~kb}$ ACAAAAGCCCACAGGAGATG-3'); tbxt $-4.0 \mathrm{~kb}$ TGGGTTTAGAATGGCAAAGG-3'); and tbxt TSS
(5'-CCAGTCCATTTTCCATGTTG-3',

5 (5'-AAAGAGTTCCTGCACCTTGG-3', 5', (5'-ACATGAGTTTGGCCTTGGTC-3', 5' (5'-CAGATGCCAGAGGAGAGAGC-3', 5'(5'-ATGAGAATGTGAGCGACACG-3', 5' (5'-TGGCTTTTAAGTTCGTCTTGG-3', 5'(5'-GATGGGGCATtAATGGACAG-3', 5'(5'-GTTAGACTCCGCTCCAGACG-3', 5'(5'-GGAAACTGCCTGTGGAAATG-3', 5'(5'-CAATGGATGCCTCTCTGGAC-3', 5'(5'-CACACCTTGGGTTTTGTTCC-3', 5'-

830 Whole-mount in situ hybridization (WMISH). WMISH was conducted using digoxigenin-labeled RNA probes. It was based on 831 previously published protocols ${ }^{56,63}$. X. tropicalis embryos were fixed in MEMFA (1x MEM and 3.7\% formaldehyde) at room 832 temperature for $1 \mathrm{~h}$. The embryos were then washed once in 1x PBS and two to three times in ethanol. Fixed and dehydrated 833 embryos were kept at $-20^{\circ} \mathrm{C}$ for $\geq 24 \mathrm{~h}$ to ensure proper dehydration before starting hybridization. Dehydrated embryos were 834 washed once more in ethanol before rehydrating them in two steps to PBT (1x PBS and 0.1\% Tween-20). Embryos were treated 
MEMFA for $20 \mathrm{~min}$ and washed three times in PBT. Embryos were transferred into baskets, which were kept in an $8 \mathrm{x} 8$ microcentrifuge tube holder sitting inside a 10x10 slot plastic box filled with PBT. Baskets were built by replacing the round bottom of 2-ml microcentrifuge tubes with a Sefar Nitex mesh. This container system was used to readily process several batches of embryos at once. These baskets were maximally loaded with 40 to $50 \mathrm{X}$. tropicalis embryos. The microcentrifuge tube holder was used to transfer all baskets at once and to submerge embryos into subsequent buffers of the WMISH protocol. Next, the embryos were incubated in $500 \mu \mathrm{l}$ hybridization buffer (50\% formamide, 5x SSC, 1x Denhardt's, $10 \mathrm{mM}$ EDTA, $1 \mathrm{mg} / \mathrm{ml}$ torula RNA, $100 \mu \mathrm{g} / \mathrm{ml}$ heparin, $0.1 \%$ Tween-20 and $0.1 \%$ CHAPS) for $2 \mathrm{~h}$ in a hybridization oven set to $60^{\circ} \mathrm{C}$. After this prehybridization step, the embryos were transferred into $500 \mu \mathrm{l}$ digoxigenin-labeled probe $(1 \mathrm{ng} / \mu \mathrm{l})$ preheated to $60^{\circ} \mathrm{C}$ and further incubated overnight at $60^{\circ} \mathrm{C}$. The pre-hybridization buffer was kept at $60^{\circ} \mathrm{C}$. The next day embryos were transferred back into the pre-hybridization buffer and incubated at $60^{\circ} \mathrm{C}$ for $10 \mathrm{~min}$. Subsequently, they were washed three times in $2 \mathrm{xSC} / 0.1 \%$ Tween20 at $60^{\circ} \mathrm{C}$ for $10 \mathrm{~min}$, twice in $0.2 \mathrm{x} \mathrm{SSC} / 0.1 \%$ Tween- 20 at $60^{\circ} \mathrm{C}$ for $20 \mathrm{~min}$ and twice in $1 \mathrm{x}$ maleic acid buffer (MAB) at room temperature for $5 \mathrm{~min}$. Next, the embryos were treated with blocking solution ( $2 \%$ Boehringer Mannheim blocking reagent in $1 \mathrm{x}$ $\mathrm{MAB})$ at room temperature for $30 \mathrm{~min}$, and incubated in antibody solution (10\% lamb or goat serum, $2 \%$ Boehringer Mannheim blocking reagent, $1 \mathrm{x} \mathrm{MAB}$ and 1:2,000 Fab fragments from polyclonal anti-digoxigenin antibodies conjugated to alkaline phosphatase) at room temperature for $4 \mathrm{~h}$. The embryos were then washed four times in 1x MAB for 10 min before leaving them in $1 \mathrm{x}$ MAB overnight at $4^{\circ} \mathrm{C}$. On the final day of the WMISH protocol, the embryos were washed another three times in 1x MAB for $20 \mathrm{~min}$ and equilibrated to working conditions of alkaline phosphatase (AP) for a total of 10 min by submerging embryos twice into AP buffer (50 mM MgCl $2,100 \mathrm{mM} \mathrm{NaCl}, 100 \mathrm{mM}$ Tris- $\mathrm{HCl} \mathrm{pH} 9.5$ and 1\% Tween-20). At this stage, the embryos were transferred to 5-ml glass vials for monitoring the progression of the AP-catalyzed colorimetric reaction. Any residual AP buffer was discarded before adding $700 \mu \mathrm{l}$ staining solution (AP buffer, $340 \mu \mathrm{g} / \mathrm{ml}$ nitro-blue tetrazolium chloride, $175 \mu \mathrm{g} / \mathrm{ml} 5$-bromo4-chloro-3'-indolyphosphate). The colorimetric reaction was developed at room temperature in the dark. Once the staining was clear and intense enough, the color reaction was stopped by two washes in 1x MAB. To stabilize and preserve morphological features, the embryos were fixed with Bouin's fixative without picric acid ( $9 \%$ formaldehyde and 5\% glacial acetic acid) at room temperature for $30 \mathrm{~min}$. Next, the embryos were washed twice in $70 \%$ ethanol/PBT to remove the fixative and residual chromogens. After rehydration to PBT in two steps, the embryos were treated with weak Curis solution $\left(1 \% \mathrm{H}_{2} \mathrm{O}_{2}, 0.5 \mathrm{x} \mathrm{SSC}\right.$ and $5 \%$ formamide) at $4^{\circ} \mathrm{C}$ in the dark overnight. Finally, the embryos were washed twice in PBS before imaging them in PBS on a thick agarose dish by light microscopy.

Antibody generation. Affinity-purified rabbit polyclonal antibodies were generated by sdix (Delaware, USA) against different amino acid (aa) sequences of $X$. tropicalis Tbx6 avoiding its highly conserved DNA binding domain (aa 95-280) among T-box proteins: aa 1-94 (\#4596), aa 304-403 (\#5061) and aa 418-517 (\#5107). Antibody \#5107 did not work (data not shown). Antibodies \#4596 and \#5061 were suitable for immunoprecipitation and Western blotting to detect endogenous Tbx6 in both X. tropicalis and X. laevis embryos (Supplementary Fig. 2k-m). Antibody \#4596 complied with ENCODE guidelines ${ }^{64}$ and thus was suitable for ChIP (Supplementary Fig. 2n).

Immunoprecipitation (IP). For each IP, 40 de-jellied embryos were collected at the developmental stage of interest. They were homogenized in $400 \mu \mathrm{l}$ ice-cold IP buffer $(50 \mathrm{mM}$ Tris- $\mathrm{HCl} \mathrm{pH}$ 7.5, $150 \mathrm{mM} \mathrm{NaCl}, 5 \mathrm{mM} \mathrm{MgCl} 2,1 \mathrm{mM}$ EDTA and $0.25 \%$ Igepal CA-630) supplemented with an EDTA-free protease inhibitor cocktail (Roche, \#11873580001). The extract was kept on ice for 5 min. Subsequently, the extract was mixed with $400 \mu 1$ 1,1,2-trichloro-1,2,2-trifluoroethane (Sigma) by vigorously inverting the tube and cleared by centrifugation at $16,000 \mathrm{~g}\left(4^{\circ} \mathrm{C}\right)$ for $5 \mathrm{~min}$. An aliquot of $20 \mu 1$ supernatant was saved as input. The remaining supernatant was incubated with $0.5 \mu 1$ antibody (anti-Sox $3^{48}$, anti-Veg $\mathrm{T}^{15}$ or anti-Tbx6; all rabbit polyclonal antibodies) at $4^{\circ} \mathrm{C}$ for $1 \mathrm{~h}$. Subsequently, each embryonic extract was supplemented with $20 \mu 1$ washed magnetic beads conjugated to sheep anti-rabbit IgG (Dynabeads M280, Invitrogen) and kept on a tube rotator at $4^{\circ} \mathrm{C}$ for another $2 \mathrm{~h}$. Next, the beads were washed three times with IP buffer. The immunoprecipitate was eluted off the beads with $20 \mu 1$ 1x SDS loading buffer (50 mM Tris-HCl pH 6.8, 2\% SDS, $6 \%$ glycerol and 5\% $\beta$-mercaptoethanol). Western blotting was carried out with TrueBlot (Rockland Immunochemicals) and 
normal horseradish peroxidase (HRP)-conjugated secondary antibodies for IP and input samples, respectively.

Western blotting. Protein samples were denatured in $1 \mathrm{x}$ (final concentration) SDS loading buffer at $70^{\circ} \mathrm{C}$ for 5 min. Denatured samples were run alongside a standard protein ladder into pre-cast gradient gels (Any kD Mini-PROTEAN TGX, BioRad) at constant $200 \mathrm{~V}$ for $1 \mathrm{~h}$. Size-separated proteins were immediately transferred onto hydrophobic Immobilon-P PVDF transfer membranes (Millipore) at constant $100 \mathrm{~V}$ for $30 \mathrm{~min}$ using standard protein electrophoresis equipment. The membranes were blocked with 5\% milk powder in PBS/0.1\% Tween-20 (MPBTw) at room temperature for 30 min. Next, the membranes were incubated at room temperature for $1 \mathrm{~h}$ with the primary antibodies diluted in MPBTw: 1:5,000 of mouse monoclonal anti- $\alpha$-tubulin (Sigma, \#T5168), 1:2,000 rabbit polyclonal anti-Sox $3^{48}, 1: 2,000$ rabbit polyclonal anti-VegT ${ }^{15}$ or 1:2,000 rabbit polyclonal antiTbx6 (\#5061). The membranes were washed three times with PBST for $10 \mathrm{~min}$ before applying 1:1,000 TrueBlot HRP-conjugated anti-rabbit IgG (Rockland Immunochemicals, \#18-8816-31) or 1:2,000 normal goat HRP-conjugated anti-mouse IgG (Thermo Fisher Scientific, \#31430) in MPBTw at room temperature. After $1 \mathrm{~h}$, the membranes were washed three times in PBTw for 10 min and once in PBS for 5 min. Finally, the membranes were treated with UptiLight US HRP WB reagent (interchim, \#58372) for $1 \mathrm{~min}$ in the dark. HRP-catalyzed chemiluminescence was detected on the ChemiDoc XRS+ system (BioRad).

Whole-mount immunohistochemistry (WMIHC). Embryos were fixed with MEMFA (0.1 M MOPS, 2 mM EGTA, 1 mM $\mathrm{MgSO}_{4}$ and 3.7\% formaldehyde) in capped 5-ml glass vials at room temperature for $2 \mathrm{~h}$, dehydrated and stored in absolute ethanol at $-20^{\circ} \mathrm{C}$ for $\geq 24 \mathrm{~h}$. For sagittal sections, fixation was stopped after $30 \mathrm{~min}$. Embryos were placed on a piece of flattened Blu-Tack (Bostik) in a large Petri dish filled with PBS to bisect them with a scalpel. Following a three-step rehydration $(50 \%, 75 \%$ and $100 \%$ PBS ) the embryos were bleached with weak Curis solution $\left(1 \% \mathrm{H}_{2} \mathrm{O}_{2}, 0.5 \mathrm{x}\right.$ SSC and $5 \%$ formamide $)$ on a light box at room temperature for $2 \mathrm{~h}$ or in the dark at $4^{\circ} \mathrm{C}$ overnight. Bleached embryos were washed three times with PBS/0.3\% Triton X-100 (PBT) and pre-incubated in blocking solution (PBS, 20\% goat or donkey serum, 2\% BSA and $0.1 \%$ Triton X-100) at $4{ }^{\circ} \mathrm{C}$ for $6 \mathrm{~h}$. The embryos were then transferred to 2-ml round-bottom microcentrifuge tubes before discarding all remaining blocking solution. The embryos were incubated at $4^{\circ} \mathrm{C}$ for 1-3 days in $50 \mu \mathrm{l}$ blocking solution containing the primary antibody at the following dilutions: 1:1,000 rabbit polyclonal anti-Sox $3^{48}, 1: 500$ rabbit polyclonal anti phospho-Smad1/5/8 (Cell Signaling, \#9511) or 1:500 goat polyclonal Smad2/3 (R\&D Systems, \#AF3797). Afterwards, the embryos were transferred back to capped 5-ml glass vials, washed three times with RIPA buffer (50 mM HEPES pH 7.5, $0.5 \mathrm{M} \mathrm{LiCl}, 1 \mathrm{mM}$ EDTA, 1\% Igepal CA-630 and 0.7\% sodium deoxycholate) for $1 \mathrm{~h}$ and once with PBT for $5 \mathrm{~min}$. Next, the embryos were incubated with the secondary HRP-conjugated antibody diluted in blocking solution at $4^{\circ} \mathrm{C}$ overnight: 1:400 goat anti-rabbit IgG-HRP (Thermo Fisher Scientific, \#G-21234) or 1:200 donkey anti-goat IgG-HRP (Santa Cruz Biotechnology, \#sc-2020). The washes of the previous day were repeated before pre-incubating the embryos in $400 \mu 1$ inactive 3,3'-diaminobenzidine tetrahydrochloride with cobalt (Sigma, \#D0426). After 1-2 min, the inactive solution was replaced with the $\mathrm{H}_{2} \mathrm{O}_{2}$-activated DAB solution. The HRP reaction was stopped after 40 sec by washing the embryos several times with PBT. In Figs. 2c and 5a,d, embryos were dehydrated in three steps to absolute methanol and cleared with Murray's clear (2:1 benzyl benzoate/benzyl alcohol) on a glass depression slide.

Deep sequencing and quality filter. All deep sequencing libraries were quality controlled: The DNA yield and fragment size distribution were determined by fluorometry and chip-based capillary or polyacrylamide gel electrophoresis, respectively. Libraries were sequenced on the Illumina platforms GAIIx or HiSeq by the Advanced Sequencing Facility of the Francis Crick Institute to produce single or paired-end reads of at least 40 bases. Next-generation capture-C libraries were sequenced on MiSeq with a read length of 150 bases. Sequencing samples and read alignment results are summarized in Supplementary Table 1. The metrics of paired-end alignments such as insert size mean and standard deviation were determined by Picard (CollectInsertSizeMetrics) from the Broad Institute (USA). 
proteins to nearby genomic DNA. Duration of fixation was determined empirically and depended mainly on the developmental stage and antibody epitopes ${ }^{65}$. Fixation was terminated by rinsing embryos three times with ice-cold $1 \%$ MMR. Where required, post-fixation embryos were dissected to select specific anatomical regions in ice-cold 1\% MMR. Fixed embryos were homogenized in CEWB1 (10 mM Tris-HCl pH 8.0, 150 mM NaCl, 1 mM EDTA, 1\% Igepal CA-630, 0.25\% sodium deoxycholate and $0.1 \%$ SDS) supplemented with $0.5 \mathrm{mM} \mathrm{DTT}$, protease inhibitors and, if using phospho-specific antibodies, phosphatase blockers $(0.5 \mathrm{mM}$ orthovanadate and $2.5 \mathrm{mM} \mathrm{NaF})$. To solubilize yolk platelets and separate them from the nuclei, the homogenate was left on ice for $5 \mathrm{~min}$ and then centrifuged at $1,000 \mathrm{~g}\left(4^{\circ} \mathrm{C}\right)$ for $5 \mathrm{~min}$. Homogenization and centrifugation was repeated once before resuspending the nuclei containing pellet in 1-3 $\mathrm{ml}$ CEWB1. Nuclear chromatin was solubilized and fragmented by isothermal focused or microtip-mediated sonication. The solution of fragmented chromatin was cleared by centrifuging at 16,000 $\mathrm{g}\left(4^{\circ} \mathrm{C}\right)$ for $5 \mathrm{~min}$. Where required, $\sim 1 \%$ of the cleared chromatin extract was set aside for the input sample (negative control). ChIP-grade antibodies were used to recognize specific chromatin features and to enrich these by coupling the antibody-chromatin complex to protein G magnetic beads (Thermo Fisher Scientific, \#10003D) and extensive washing. These steps were carried out at $4^{\circ} \mathrm{C}$. The beads were washed twice in CEWB1, twice in WB2 (10 mM Tris-HCl pH 8.0, $500 \mathrm{mM} \mathrm{NaCl}, 1 \mathrm{mM}$ EDTA, $1 \%$ Igepal CA-630, 0.25\% sodium deoxycholate and 0.1\% SDS), twice in WB3 (10 mM Tris-HCl pH 8.0, $250 \mathrm{mM} \mathrm{LiCl,} 1 \mathrm{mM} \mathrm{EDTA}$, 1\% Igepal CA-630 and 1\% sodium deoxycholate) and once in TEN (10 mM Tris-HCl pH 8.0, $150 \mathrm{mM} \mathrm{NaCl}$ and 1 mM EDTA). ChIP was eluted off the beads twice with $100 \mu \mathrm{l}$ SDS elution buffer $(50 \mathrm{mM}$ Tris-HCl pH 8.0, $1 \mathrm{mM} \mathrm{EDTA}$ and $1 \%$ SDS $)$ at $65^{\circ} \mathrm{C}$. ChIP eluates were pooled before reversing DNA-protein cross-links. Input (filled up to $200 \mu 1$ with SDS elution buffer) and ChIP samples were supplemented with $10 \mu 15 \mathrm{M} \mathrm{NaCl}$ and incubated at $65^{\circ} \mathrm{C}$ for $6-16 \mathrm{~h}$. Samples were treated with proteinase $\mathrm{K}$ (Thermo Fisher Scientific, \#AM2548) and RNase A (Thermo Fisher Scientific, \#12091021) to remove any proteins and RNA from the co-immunoprecipitated DNA fragments. The DNA was purified with phenol:chloroform:isoamyl alcohol (25:24:1, $\mathrm{pH}$ 7.9) using 1.5-ml Phase Lock Gel Heavy microcentrifuge tubes (VWR) for phase separation and precipitated with 1/70 volume of $5 \mathrm{M} \mathrm{NaCl}, 2$ volumes of absolute ethanol and $15 \mu \mathrm{g}$ GlycoBlue (Thermo Fisher Scientific, \#AM9516). After centrifugation, the DNA pellet was air-dried and dissolved in $11 \mu$ l elution buffer $(10 \mathrm{mM}$ Tris- $\mathrm{HCl} \mathrm{pH} \mathrm{8.5).} \mathrm{The} \mathrm{DNA} \mathrm{concentration} \mathrm{was} \mathrm{determined}$ on a fluorometer using high-sensitivity reagents for double-stranded DNA $(10 \mathrm{pg} / \mu 1$ to $100 \mathrm{ng} / \mu \mathrm{l})$.

The following antibodies were used for ChIP: rabbit polyclonal anti- $\beta$-catenin (Santa Cruz Biotechnology, \#H-102), rabbit polyclonal anti-H3K4me1 (Abcam, \#ab8895), rabbit polyclonal anti-HA (Abcam, \#ab9110), mouse monoclonal anti-RNAPII (Covance, \#MMS-126R), rabbit polyclonal anti-phospho-Smad1/5/8 (Cell Signaling, \#9511), goat polyclonal anti-Smad2/3 (R\&D Systems, \#AF3797), rabbit polyclonal anti-Sox $3^{48}$, rabbit polyclonal anti-Tbx6 (\#4596) and rabbit polyclonal anti-VegT ${ }^{15}$.

Quantitative PCR (qPCR). DNA was amplified in technical duplicates with SYBR Green I master mix (Roche, \#04707516001) on a Light Cycler 480 II (Roche) cycling 55 -times between $94^{\circ} \mathrm{C}, 60^{\circ} \mathrm{C}$ and $72^{\circ} \mathrm{C}$ with each temperature step running for 10 sec and switching at $+4.8^{\circ} \mathrm{C} / \mathrm{sec}$ and $-2.5^{\circ} \mathrm{C} / \mathrm{sec}$. At the end $\mathrm{qPCR}$ reactions were heated from $65^{\circ} \mathrm{C}$ to $97^{\circ} \mathrm{C}$ with a gradual increase of $0.11^{\circ} \mathrm{C} / \mathrm{sec}$ (melting curve) to ensure only fluorescence was collected from one specific amplicon. ChIP-qPCR results were based on absolute quantification using an eight-point standard curve of three-fold dilutions of $\sim 1 \%$ input DNA (Supplementary Fig. 2d,g,i,j,n). RT-qPCR results were normalized to the housekeeping gene $o d c l$ (Figs. $4 \mathbf{h}$ and $\mathbf{7 d}$ and Supplementary Figs. 10d) or rpl8 (Supplementary Fig. 8b), and scaled relative to control embryos using the $2^{-\Delta \Delta C t}$ method $^{66}$. The threshold cycle $\left(C_{t}\right)$ was derived from the maximum acceleration of SYBR fluorescence (second derivative maximum method).

ChIP-Seq library preparation. 0.5 to $10 \mathrm{ng}$ ChIP DNA or $5 \mathrm{ng}$ input DNA were used to prepare single (only Tbx6 ChIP-Seq) or indexed paired-end libraries as previously described ${ }^{65,67-69}$. Institute, UK) to trim off low-quality bases (default Phred score of 20, i.e. error probability was 0.01 ) and adapter contamination from the 3' end. Processed reads were aligned to the $X$. tropicalis genome assembly v7.1 (and v9.1 for some ChIP-Seq data) 
format ${ }^{71}$ with redundant reads being removed (makeTagDirectory -single -tbp 1 -unique -mapq 10 -fragLength 175 -totalReads all). Only uniquely aligned reads (i.e. MAPQ $\geq 10$ ) were processed. We pooled all input alignments from various developmental stages. This created a comprehensive mappability profile that covered $\sim 400$ million unique base pair positions. All chromatin profiles were position-adjusted and normalized to the effective total of 1 million aligned reads including multimappers (counts per million aligned reads, CPM). Agreeing biological replicates according to ENCODE guidelines ${ }^{64}$ were subsequently merged. For stage $10^{+}$(early gastrula stage) $\beta$-catenin and Smad2 ChIP-Seq, external datasets ${ }^{25,72,73}$ were used as biological replicates. HOMER's peak caller was used to identify transcription factor binding sites by virtue of ChIP-enriched read alignments (hereafter called peaks): findpeaks -style factor -minDist 175 -fragLength 175 -inputFragLength 175 -fdr 0.001 -gsize $1.435 \mathrm{e} 9$-F 3 -L 1 -C 0.97. This means that both ChIP and input alignments were extended 3' to $175 \mathrm{bp}$ for the detection of significant (0.1\% FDR) peaks being separated by $\geq 175 \mathrm{bp}$. The effective size of the $X$. tropicalis genome assembly v7.1 was set to $1.435 \mathrm{billion} b \mathrm{~b}$, an estimate obtained from the mappability profile. These peaks showed equal or higher tag density than the surrounding $10 \mathrm{~kb}, \geq 3-$ fold more tags than the input and $\geq 0.97$ unique tag positions relative to the expected number of tags. To detect focal RNAPII recruitment to pCRMs and avoid calling peaks within broad regions of RNAPII reflecting transcript elongation, the threshold of focal ratio and local enrichment within $10 \mathrm{~kb}$ was elevated to 0.6 and 3 (-L 3), respectively. To further eliminate any false positive peaks, we removed any peaks with $<0.5$ (TFs including signal mediators) or $<1$ (RNAPII) CPM and those falling into blacklisted regions showing equivocal mappability due to genome assembly errors, gaps or simple/tandem repeats. Regions of equivocal mappability were identified by a two-fold lower (poor) or three-fold higher (excessive) read coverage than the average detected in 400-bp windows sliding at 200-bp intervals through normalized ChIP input and DNase-digested naked genomic DNA. All identified regions $\leq 800$ bp apart were subsequently merged. Gap coordinates were obtained from the Francis Crick mirror site of the UCSC genome browser (http://genomes.crick.ac.uk). Simple repeats were masked with RepeatMasker v4.0.6 $6^{74}$ using the crossmatch search engine v1.090518 and the following settings: RepeatMasker -species "xenopus silurana tropicalis" -s -xsmall. Tandem repeats were masked with Jim Kent's trfBig wrapper script of the Tandem Repeat Finder v4.09 ${ }^{75}$ using the following settings: weight for match, 2; weight for mismatch, 7; delta, 7; matching probability, 80; indel probability, 10; minimal alignment score, 50; maximum period size, 2,000; and longest tandem repeat array (-1), 2 [million bp]. The multi-genome sequence conservation track (phastCons) for $X$. tropicalis genome assembly v9.1 was obtained from Xenbase ${ }^{76}$. The following eleven vertebrate species were used to evaluate sequence similarity: X. tropicalis, X. laevis, Nanorana parkeri (High Himalaya Frog), Fugu rubripes (Japanese Pufferfish), Chrysemys picta (Painted Turtle), Gallus gallus (Chicken), Anolis carolinensis (Green Anole lizard), Monodelphis domestica (Gray Short-tailed Opossum), Canis lupus familiaris (dog), mouse and human. pCRMs with a phastCons average $\geq 0.4$ were considered 'conserved'.

Poly(A) RNA-Seq. Libraries were made from $\sim 1 \mu \mathrm{g}$ total RNA by following the low-sample protocol of the TruSeq RNA sample preparation guide version 2 with a few modifications. First, $1 \mu \mathrm{l}$ cDNA purified after second strand synthesis was quantified on a fluorometer using high-sensitivity reagents for double-stranded DNA (10 pg/ $/ 1$ to $100 \mathrm{ng} / \mu \mathrm{l})$. By this stage, the yield was $\sim 10 \mathrm{ng}$. Second, the numbers of PCR cycles were adjusted to the detected yield of cDNA to avoid products of over-amplification such as chimera fragments: 7 ( 20 ng), $8(\sim 10 \mathrm{ng}), 9(\sim 5 \mathrm{ng})$ and $12(\sim 1 \mathrm{ng})$.

1006

RNA-Seq read alignment. Paired-end reads were aligned to the $X$. tropicalis genome assembly v7.1 using STAR v2.5.3a ${ }^{77}$ with default settings (Supplementary Table 1) and a revised version of gene models v $7.2^{50}$ to improve mapping accuracy across splice junctions. The alignments were sorted by read name using the sort function of samtools v1.3.1 ${ }^{78}$. Exon and intron counts (-t 'exon;intron') were extracted from unstranded (-s 0) alignment files using VERSE v0.1.5 $5^{79}$ in featureCounts (default) mode (-z 0). Intron coordinates were adjusted to exclude any overlap with exon annotation. For visualization, genomic BAM files of biological replicates were merged using samtools v1.3.1 and converted to the bigWig format. These genome tracks were normalized to the 
1015 Differential gene expression analysis. Differential expression analysis was performed with both raw exon and intron counts 1016 excluding those belonging to ribosomal and mitochondrial RNA using the Bioconductor/R package DESeq2 v1.16.1 ${ }^{81}$. In an effort 1017 to find genes with consistent fold changes over time, p-values were generated according to a likelihood ratio test reflecting the 1018 probability of rejecting the reduced ( developmental stage) over the full $(\sim$ developmental stage + condition) model. Resulting p1019 values were adjusted to obtain false discovery rates (FDR) according to the Benjamini-Hochburg procedure with thresholds on Cook's distances and independent filtering being switched off. Equally, regional expression datasets ${ }^{51}$ without time series were subjected to likelihood ratio tests with reduced $(\sim 1)$ and full ( condition) models for statistical analysis. Fold changes of intronic and exonic transcript levels were calculated for each developmental stage and condition using the mean of DESeq2-normalized read counts from biological replicates. Both intronic and exonic datasets were filtered for $\geq 10$ DESeq2-normalized read counts detected at least at one developmental stage in all uninjected or DMSO-treated samples. Gene-specific fold changes were removed at developmental stages that yielded $<10$ normalized read counts in corresponding control samples. Next, the means of intronic and exonic fold changes were calculated across developmental stages. The whole dataset was confined to 3,687 genes for which at least $50 \%$ reductions (FDR $\leq 10 \%$ ) in exonic (default) or intronic counts could be detected in $\alpha$-amanitin-injected embryos. Regional expression was based on exonic read counts by default unless the intronic fold changes were significantly (FDR $\leq 10 \%)$ larger than the exonic fold changes (Supplementary Table 9).

Analyzing ribosome footprinting and mass-spectrometry data. The ribosome footprinting reads were trimmed 5' by 8 bases and 3' by as many bases overlapping with the adapter sequence 5'-TCGTATGCCGTCTTCTGCTTG-3' from the 5' end. All trimmed reads between 27 to 32 bases in length were aligned first to ribosomal RNA as listed in the SILVA rRNA database ${ }^{82}$ using Bowtie v1.0.1 ${ }^{83}$ with the following parameters: --seedlen 25 (seed length) --seedmms 1 (number of mismatches allowed in the seed) --un (unaligned reads were reported). All non-aligned (rRNA-depleted) reads were mapped to the gene model 6.0 of $X$. laevis using Tophat v2.0.10 84 --no-novel-juncs (spliced reads must match splice junctions of gene model 6.0) --no-novel-indels (indel detetion disabled) --segment-length 15 (minimal length of read fragment to be aligned) -GTF v6.0.gff3 --prefilter-multihits (reads aligned first to whole genome to exclude reads aligning $>10$ times) --max-multihits 10. Alignment files were converted to the HOMER's tag density format ${ }^{71}$ before retrieving reads for each CDS per kilobase and one million mapped reads (rpkm) using HOMER's perl script analyzeRNA.pl. This read count table was merged with a published list of estimated protein concentrations $(\mathrm{nM})$ in the $X$. laevis egg using mass-spectrometry ${ }^{12}$ (Supplementary Table 4). Chromatin-associated proteins regulating RNAPII-mediated transcription were filtered based on human (version 2.0, 09/2014) and Xenbase-released gene ontology (GO) associations. The genes associated with any of the following GO terms were verified with UniProt (UniProt Consortium, 2015) whether chromatin binding is supported by functional evidence: chromatin (GO:0000785), DNA binding (GO:0003677), DNAtemplated regulation of transcription (GO:0006355), sequence-specific DNA binding and TF activity (GO:0003700) and DNA replication (GO:0006260). We separated specific TFs from all other chromatin binding proteins to form three categories: (1) sequence-specific DNA binding factors, (2) other RNAPII-regulating factors and (3) remaining genes, whereby genes associated with DNA repair (GO:0006281) and RNAPIII (GO:0006383) were moved to the third category. blastomeres made it impossible to employ ATAC-Seq ${ }^{11}$ on whole Xenopus embryos before the onset of gastrulation. Therefore, DNase-Seq was adapted to early Xenopus embryos with a novel approach to select small DNase-digested DNA fragments. Ultracentrifugation- or gel electrophoresis-mediated size selection ${ }^{85,86}$ was replaced by two rounds of solid phase immobilization (SPI) to remove long inaccessible DNA from short accessible DNA. Wide-bore pipette tips were used for the resuspensions and the transfers of samples from the second homogenization step until after SPI to minimize fragmentation of high-molecular DNA. Approximately 250 de-jellied mid-blastula embryos were collected in 2-ml round-bottom microcentrifuge tubes and homogenized in $2 \mathrm{ml}$ ice-cold LB-DNase buffer $(15 \mathrm{mM}$ Tris- $\mathrm{HCl} \mathrm{pH}$ 8.0, $15 \mathrm{mM} \mathrm{NaCl}, 60 \mathrm{mM} \mathrm{KCl}, 1 \mathrm{mM}$ EDTA, $0.5 \mathrm{mM}$ EGTA and 0.5 $\mathrm{mM}$ spermidine) supplemented with $0.05 \%$ Igepal CA-630. This lysate was left on ice for 3 min before centrifuging at 1,000 $\mathrm{g}$ 
1060 LB-DNase buffer (without Igepal CA-630) before centrifuging again at 1,000 $\mathrm{g}\left(4^{\circ} \mathrm{C}\right)$ for 2 min. After discarding the supernatant, 1061 the pellet was resuspended at room temperature in $600 \mu 1$ LB-DNase buffer (without Igepal CA-630) with $6 \mathrm{mM} \mathrm{CaCl}_{2}$. The sample was distributed equally to two $1.5-\mathrm{ml}$ microcentrifuge tubes, one for probing chromatin accessibility with DNase and the other one for creating a reference profile of DNase-digested naked genomic DNA. Approximately 0.1 U recombinant DNase I (Roche, \#04716728001) was added to one aliquot. Both samples were incubated at $37^{\circ} \mathrm{C}$ for 8 min before adding $300 \mu 1 \mathrm{STOP}$ buffer $(0.1 \%$ SDS, $100 \mathrm{mM} \mathrm{NaCl}, 100 \mathrm{mM}$ EDTA and $50 \mathrm{mM}$ Tris-HCl pH 8.0) including $80 \mu \mathrm{g}$ RNase A (Thermo Fisher Scientific, \#12091021), $333 \mathrm{nM}$ spermine and $1 \mu \mathrm{M}$ spermidine. The tubes were inverted gently to mix samples before incubating them at $55^{\circ} \mathrm{C}$ for $15 \mathrm{~min}$. Next, $200 \mu \mathrm{g}$ proteinase $\mathrm{K}$ (Thermo Fisher Scientific, \#AM2548) were added and the tubes were inverted gently to mix the samples again. After $2 \mathrm{~h}$ at $55^{\circ} \mathrm{C}$, the digests were transferred to pre-spun 1.5-ml Phase Lock Gel Heavy microcentrifuge tubes (VWR). $600 \mu 1$ phenol:chloroform:isoamylalcohol (25:24:1, pH 7.9) were added to the digests. The tubes were shaken gently and then centrifuged at $1,500 \mathrm{~g}$ (room temperature) for $4 \mathrm{~min}$. The top phase was transferred to a fresh 2-ml microcentrifuge tube and mixed with $60 \mu 13 \mathrm{M}$ sodium acetate $(\mathrm{pH} \mathrm{5.2)}$ and $1.2 \mathrm{ml}$ absolute ethanol to precipitate the genomic DNA. The precipitation was stored at $-20^{\circ} \mathrm{C}$ overnight and centrifuged at $16,000 \mathrm{~g}\left(4^{\circ} \mathrm{C}\right)$ for $30 \mathrm{~min}$. The supernatant was discarded and the DNA pellet washed by adding $500 \mu \mathrm{l}$ ice-cold $80 \%$ ethanol and centrifuging at $16,000 \mathrm{~g}\left(4^{\circ} \mathrm{C}\right)$ for 3 min. The ethanol was discarded and the DNA pellet was air-dried at room temperature for $10 \mathrm{~min}$. After that, the DNA pellet was left on ice to dissolve in $27 \mu$ elution buffer $(10 \mathrm{mM}$ Tris- $\mathrm{HCl} \mathrm{pH} 8.5)$ for $20 \mathrm{~min}$. To remove any residual RNA, $10 \mu \mathrm{g}$ RNase A were added to the DNA. $1 \mu 1$ was used to determine the DNA fragment size distribution by gel electrophoresis. On a $0.6 \%$ TAE agarose gel, a smear of very high molecular DNA was visible as expected from previous DNase experiments ${ }^{85,87}$. Genomic DNA to the amount of 20 untreated mid-blastula embryos was digested with $0.3 \mathrm{U}$ DNase I at $37^{\circ} \mathrm{C}$ for $5 \mathrm{~min}$ following the same steps as described above, which generated a low-molecular smear of DNA fragments. Next, $70 \mu 1$ AMPure XP beads (Beckman Coulter, \#A63880) per DNase sample were equilibrated to room temperature for $10 \mathrm{~min} .22 .5 \mu 1$ AMPure XP beads ( $0.9 \mathrm{x}$ of the volume) were added to the DNA sample without pipetting up and down achieving a final polyethylene glycol concentration of $\sim 9.5 \%$. After 3 min, by which time high-molecular DNA causes beads to coalesce, the tubes were clipped into a magnetic stand for microcentrifuge tubes. After $3 \mathrm{~min}$ or until the beads were separated from the supernatant, the latter was transferred to a 96 -well microplate (350- $\mu 1$ round wells with V-shaped bases). $47.5 \mu 1$ elution buffer and $43 \mu 1$ AMPure XP beads were added sequentially and mixed gently by slowly pipetting up and down. After $3 \mathrm{~min}$, the plate was transferred to a magnetic stand for 96-well plates. Once the beads have completely separated from the suspension, the supernatant was transferred to a pre-spun 1.5-ml Phase Lock Gel Heavy tube. 162 $\mu 1$ elution buffer and $300 \mu \mathrm{l}$ phenol:chloroform:isoamylalcohol (25:24:1, pH 7.9) were added. The tubes were shaken gently and then centrifuged at $1,500 \mathrm{~g}$ (room temperature) for $4 \mathrm{~min}$. The top phase was transferred to a $1.5-\mathrm{ml}$ low-retention microcentrifuge tube and mixed with $30 \mu 13 \mathrm{M}$ sodium acetate (pH 5.2) and $900 \mu 1$ absolute ethanol to precipitate the DNA as outlined above. The DNA pellet was dissolved in $12 \mu 1$ elution buffer. The concentrations of the DNA samples were determined on a fluorometer using

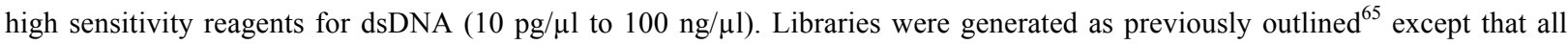
cleaning steps were executed with 0.2x more AMPure XP beads.

Post-sequencing analysis of DNase-Seq. Single and paired-end reads of maximal 50 bases were processed using trim_galore v0.4.2 from the Babraham Institute (UK) to trim off low-quality bases (default Phred score of 20, i.e. error probability was 0.01 ) and adapter contamination from the 3' end. Processed reads were aligned to the $X$. tropicalis genome v7.1 and v9.1 using Bowtie2 v2.2.9 $9^{70}$ with default settings apart from - X (fragment length), which was reduced to $250 \mathrm{bp}$ for paired-end reads. Alignments were sorted by genomic coordinates and only those with a quality score of $\geq 10$ were retained using samtools v1.3.18. Duplicates were removed using Picard (MarkDuplicates) from the Broad Institute (USA). Paired-end alignments were dissociated using hex flags (-f 0x40 or 0x80) of samtools view. Single alignments were converted to HOMER's tag density format ${ }^{71}$ (makeTagDirectory single -unique -fragLength 100 -totalReads all). DNase hypersensitive sites were identified using the following or otherwise default parameters of HOMER's peak calling: findpeaks -style factor -minDist 100 -fragLength 100 -inputFragLength 100 -fdr 0.001 gsize 1.435e9 -F 3 -L 1 -C 0.97. This means that alignments of DNase-digested chromatin fragments (or naked genomic DNA fragments considered here as 'input') were extended 3' to $100 \mathrm{bp}$ from the DNase cleavage site to detect significant (0.1\% FDR) 
DNase-enriched read alignments (hereafter called peaks) being separated by $\geq 100 \mathrm{bp}$. The effective size of the $X$. tropicalis genome assembly v7.1 was set to 1.435 billion bp, an estimate obtained from the mappability of ChIP input reads. These peaks showed equal or higher tag density than the surrounding $10 \mathrm{~kb}$, at least three-fold more tags than the input and $\geq 0.97$ unique tag positions relative to the expected number of tags. Peaks falling into blacklisted regions (see Post-sequencing analysis of ChIP-Seq) were removed. The profiles of DNase-probed chromatin accessibility were position-adjusted and normalized to the effective total of 1 million aligned reads including multimappers. Genomic coordinates of accessible pCRMs are listed in Supplementary Tables 3 and $\mathbf{1 0}$ (mPouV/Sox3 LOF experiment).

1112

1113 Next-generation capture-C. About 500 mid-blastula embryos per condition were collected in 9-ml capped glass vials and briefly washed once with 1\% MMR. The embryos were then fixed with 1\% formaldehyde (Sigma, \#F8775) in $9 \mathrm{ml} 1 \%$ MMR at room temperature for $40 \mathrm{~min}$. The fixation reaction was terminated by briefly rinsing the embryos three times with ice-cold $1 \%$ MMR. The embryos were aliquoted into 2-ml round-bottom microcentrifuge tubes in batches of $\sim 250$ embryos (filling the volume of $\sim 250 \mu 1$ water). The supernatant was removed before equilibrating embryos in $250 \mu 1$ ice-cold HEG buffer (50 mM HEPES pH 7.5, 1 mM EDTA and 20\% glycerol). Once the embryos settled to the bottom of the tube as much liquid as possible was discarded. The aliquots were snap-frozen in liquid nitrogen and stored at $-80^{\circ} \mathrm{C}$.

For each chromatin conformation capture (3C) experiment, $10 \mathrm{ml}$ chromatin extraction buffer CEB-3C (10 mM Tris- $\mathrm{HCl} \mathrm{pH}$ 8.0, $150 \mathrm{mM} \mathrm{NaCl}, 1 \mathrm{mM}$ EDTA, 1\% Igepal CA-630, 0.25\% sodium deoxycholate and $0.2 \%$ SDS) were supplemented with a

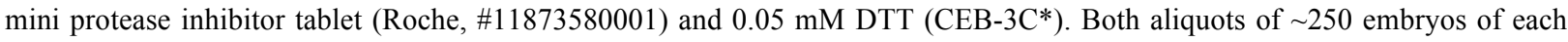
condition were homogenized in $2 \mathrm{ml}$ ice-cold CEB-3C* The tubes were kept on ice for $5 \mathrm{~min}$ before centrifuging at $1,000 \mathrm{~g}\left(4^{\circ} \mathrm{C}\right)$ for $2 \mathrm{~min}$. The supernatants were discarded and the pellet resuspended in $0.5 \mathrm{ml}$ ice-cold CEB-3C*. The resuspensions of each condition were pooled and then equally divided to two 50-ml conical tubes and each filled up with CEB-3C (without protease inhibitors and DTT) to $50 \mathrm{ml}$. The embryonic extracts were incubated at $37^{\circ} \mathrm{C}$ for $1 \mathrm{~h}$ in a hybridization oven with the tubes rotating inside a hybridization bottle. The tubes were then centrifuged at 1,000 $\mathrm{g}$ (room temperature) for $5 \mathrm{~min}$. The supernatants were discarded and the pellets resuspended in $50 \mathrm{ml}$ double-distilled water. The tubes were centrifuged again at 1,000 g (room temperature) for $5 \mathrm{~min}$. The supernatants were discarded and each pellet was resuspended in $300 \mu 1$ double-distilled water. The resuspensions containing cross-linked nuclei of each condition were pooled in a 1.5-ml microcentrifuge tube. The samples were then digested with $400 \mathrm{U} D p n \mathrm{II}$ (NEB, \#R0543) in a total volume of $800 \mu 1$ containing 1x DpnII reaction buffer. The digest was incubated overnight in a thermomixer set to $37^{\circ} \mathrm{C}$ and $1,400 \mathrm{rpm}$. Of note, any residual yolk platelets in the solution did not interfere with the restriction digest. The next day, the digest was supplemented with $200 \mathrm{U} D p n \mathrm{II}$ and incubated at $37^{\circ} \mathrm{C}$ and $1,400 \mathrm{rpm}$ for another 6-8 h. After that, DpnII was heat-inactivated at $65^{\circ} \mathrm{C}$ for $20 \mathrm{~min}$.

To verify the degree of chromatin digestion, an aliquot of $50 \mu 1$ from each condition was transferred into separate 1.5 -ml microcentrifuge tubes and processed as follows: First, any remaining RNA was degraded by incubating the aliquot with $20 \mu \mathrm{g}$ RNase A (Thermo Fisher Scientific, \#12091021) at $37^{\circ} \mathrm{C}$ for $30 \mathrm{~min}$. Second, proteins were digested by incubating the aliquot mixed with $50 \mu \mathrm{l}$ SDS elution buffer (50 mM Tris $\mathrm{pH} 8.0,1 \mathrm{mM}$ EDTA and 1\% SDS) and $20 \mu \mathrm{g}$ proteinase K (Thermo Fisher Scientific, \#AM2548) at $65^{\circ} \mathrm{C}$ in a hybridization oven overnight. Third, the DNA was purified and ethanol precipitated: $300 \mu 1 \mathrm{TE}$ pH 8.0 were added to the digest before transferring it to pre-spun 1.5-ml Phase Lock Gel Heavy microcentrifuge tubes (VWR). The diluted digests were mixed vigorously with $400 \mu \mathrm{l}$ (1 volume) phenol:chloroform:isoamylalcohol (25:24:1, pH 7.9) before centrifuging at 16,000 $\mathrm{g}$ (room temperature) for $1 \mathrm{~min}$. The top (aqueous) phases were transferred into 1.5-ml microcentrifuge tubes and mixed with $40 \mu 1$ sodium acetate ( $\mathrm{pH} 5.2$ ) and $800 \mu 1$ absolute ethanol and $15 \mu \mathrm{g}$ GlycoBlue (Thermo Fisher Scientific, \#AM9516). The DNA precipitations were kept at $-80^{\circ} \mathrm{C}$ for $30 \mathrm{~min}$ before centrifuging at $16,000 \mathrm{~g}\left(4^{\circ} \mathrm{C}\right)$ for $30 \mathrm{~min}$. The supernatants were discarded without disturbing the DNA pellets which were subsequently washed once with $400 \mu 1$ ice-cold $80 \%$ ethanol. The tubes were centrifuged at $16,000 \mathrm{~g}\left(4^{\circ} \mathrm{C}\right)$ for $30 \mathrm{~min}$ before discarding the supernatants. The DNA pellets were airdried for $10 \mathrm{~min}$ and resuspended in $10 \mu \mathrm{l}$ elution buffer $(10 \mathrm{mM}$ Tris- $\mathrm{HCl} \mathrm{pH} 8.5)$. The DNA concentrations were determined 
chromatin with DpnII caused extensive fragmentation of the genomic DNA such that no high-molecular DNA fragment bands $(>10 \mathrm{~kb})$ were visible.

1151 The chromatin digests were processed for proximity ligation by adding 240 U T4 DNA ligase (Thermo Fisher Scientific, \#EL0013) in a total volume of $1.2 \mathrm{ml}$ containing 1x T4 DNA ligase buffer including $5 \mathrm{mM}$ ATP. The ligation reactions were incubated at $16^{\circ} \mathrm{C}$ and $1,400 \mathrm{rpm}$ for $\geq 16 \mathrm{~h}$ before centrifuging at $16,000 \mathrm{~g}\left(4^{\circ} \mathrm{C}\right)$ for $1 \mathrm{~min}$. The supernatants were discarded. 200 $\mu 1$ elution buffer and $40 \mu \mathrm{g}$ RNase A were added to the pellets to degrade any residual RNA at $37^{\circ} \mathrm{C}$ and $1,400 \mathrm{rpm}$ for $30 \mathrm{~min}$. Proteins were digested by adding $200 \mu \mathrm{l}$ SDS elution buffer and $160 \mu \mathrm{g}$ proteinase $\mathrm{K}$ and incubating at $65^{\circ} \mathrm{C}$ in a hybridization oven overnight. The digest was transferred to pre-spun 1.5-ml Phase Lock Gel Heavy tubes and processed as described in the previous paragraph to purify and precipitate ligated genomic DNA. The air-dried DNA pellet was dissolved in $20 \mu 1$ elution buffer and quality controlled as above. Proximity ligation of DpnII-digested chromatin fragments massively increased the size of DNA fragments to the range of high molecular weight $(>10 \mathrm{~kb})$ representing the $3 \mathrm{C}$ library. The concentration was measured on a fluorometer with broad range concentration $(5-500 \mathrm{ng} / \mu \mathrm{l})$ reagents.

About $10-15 \mu \mathrm{g}$ of the $3 \mathrm{C}$ libraries were diluted with elution buffer to the total volume of $130 \mu \mathrm{l}$ and transferred to a designated glass vial (microTUBE) for isothermal focused sonication (Covaris). The following settings of the focused ultrasonicator Covaris S220 were used to shear the libraries to an average DNA fragment length of 200 bp: duty cycle, $10 \%$; intensity, 5; cycles per burst, 200; duration, $60 \mathrm{sec}$ in frequency sweeping mode. Sonication was run with 6 cycles in batch mode. The degree of DNA fragmentation was verified by gel electrophoresis and the DNA concentration was measured on a fluorometer with broad range concentration reagents.

Approximately 2-4 $\mu \mathrm{g}$ of the DNA fragments were converted into next-generation paired-end libraries using the enzymes of the

1168 KAPA Hyper Prep Kit (Kapa Biosystems, \#KR0961) and TruSeq adaptors (Illumina). The end-repair and A-tailing reactions were set up according to the manufacturer's instructions and incubated at $20^{\circ} \mathrm{C}$ for $1 \mathrm{~h}$ followed by $30 \mathrm{~min}$ at $65^{\circ} \mathrm{C}$. End-repaired and A-tailed DNA fragments were ligated to $150 \mathrm{pmol}$ TruSeq adaptors of index 6 and 12 , respectively, at $20^{\circ} \mathrm{C}$ for $1 \mathrm{~h}$. The DNA was purified using 0.8x volume of AMPure XP beads (Beckman Coulter, \#A63880) and amplified in a total volume of $100 \mu 1$ using 50 pmol of each TruSeq PCR primer, KAPA HiFi HotStart ReadyMix (Kapa Biosystems, \#KK2602) and the following PCR conditions: $98^{\circ} \mathrm{C}$ for $45 \mathrm{sec}$ followed by 3 cycles $\left(98^{\circ} \mathrm{C}\right.$ for $15 \mathrm{sec}, 98^{\circ} \mathrm{C}$ for $30 \mathrm{sec}$ and $72^{\circ} \mathrm{C}$ for $\left.30 \mathrm{sec}\right)$ and a final elongation step of $1 \mathrm{~min}$ at $72^{\circ} \mathrm{C}$. The lid temperature was set to $105^{\circ} \mathrm{C}$. The PCR reactions were cleaned up with $100 \mu 1$ AMPure XP beads and eluted in $14 \mu$ l elution buffer. The integrity of the library DNA was verified on a chip-based capillary electrophoresis system, an $8 \%$ TBE polyacrylamide gel or a $2 \%$ TAE agarose gel. DNA concentration was measured on a fluorometer with broad range concentration reagents. The DNA yield was $2-4 \mu \mathrm{g}$.

To capture the genomic regions of interest (viewpoints), 5'-biotinylated oligonucleotides of 120 bases (hereafter called probes)

(Supplementary Table 10) were designed for each viewpoint as follows: The viewpoints were DpnII fragments of 300-3,300 bp which were overlapping gene promoters or located $<1 \mathrm{~kb}$ from them. Each probe matched the terminal sequence of the same DNA fragment strand including the DpnII restriction site GATC. The probe sequence was examined using BLAT to check whether it is unique and did not partially match any other genomic regions. Furthermore, CENSOR v4.2.29 $9^{88}$ and RepeatMasker v4.0.6 $6^{74}$ programs were run to discard any probes that contained repeats. Because of these design restrictions, six of the selected 30 gene promoters were captured with only one probe. All the probes were purchased as desalted oligomers (4 nmol) from IDT (ultramer technology) and reconstituted in molecular-grade water to $10 \mu \mathrm{M}$. The probes were mixed in equimolar quantities and diluted to $2.9 \mathrm{nM}$ such that $4.5 \mu 1$ contained 13 fmol of oligomers.

The capture was performed with the SeqCap Hybridization and Wash Kit (Roche, \#05634253001) and SeqCap HE-Oligo Kit A (Roche, \#06777287001) as follows. Exactly $1 \mu \mathrm{g}$ of each TruSeq library with index 6 and 12 was mixed with $1 \mathrm{nmol}$ TruSeq universal blocking oligonucleotide and $0.5 \mathrm{nmol}$ blocking oligonucleotides specific to TruSeq index 6 and 12 in a 1.5 -ml lowretention microcentrifuge tube. Of note, Cot-1 DNA commonly used to mask repetitive DNA proved to be unnecessary here in reducing non-specific hybridization. The mixture of library and blocking oligonucleotides was dried in a vacuum centrifuge before adding $7.5 \mu 12 x$ hybridization buffer (vial 5) and $3 \mu 1$ hybridization component A (vial 6). This mixture was vortexed for 10 sec, centrifuged at $16,000 \mathrm{~g}$ for $10 \mathrm{sec}$ and incubated at $95^{\circ} \mathrm{C}$ for $10 \mathrm{~min}$. In the meantime, $4.5 \mu \mathrm{l}(13 \mathrm{fmol})$ of the equimolar probe 
mixture were transferred to a PCR tube and incubated at $47^{\circ} \mathrm{C}$ in a PCR machine with the volume and the lid temperature set to $15 \mu 1$ and $57^{\circ} \mathrm{C}$, respectively. Upon denaturation, the libraries and blocking oligonucleotides were added to the probes without removing either tubes from the heating block or the PCR machine. The hybridization mixture was incubated at $47^{\circ} \mathrm{C}$ for $64-72 \mathrm{~h}$. The wash buffers were prepared according to the manufacturer's instructions to make $1 \mathrm{x}$ working solutions for one single capture experiment. The stringent wash buffer and wash buffer I were pre-warmed to $47^{\circ} \mathrm{C}$ and $50 \mu 1 \mathrm{M} 270$ streptavidin-conjugated magnetic beads (Thermo Fisher Scientific, \#65305) were transferred into a 1.5-ml low-retention microcentrifuge tube to let them equilibrate to room temperature for $10 \mathrm{~min}$. The beads were washed twice with $200 \mu \mathrm{l}$ bead wash buffer. Immediately after the final wash, the hybridization mixture was directly added to the beads and vortexed for $10 \mathrm{sec}$. The sample was incubated for 45 min in a thermomixer set to $47^{\circ} \mathrm{C}$ and $1,100 \mathrm{rpm}$. The beads were washed by adding $100 \mu 1$ pre-warmed wash buffer I and vortexing for $10 \mathrm{sec}$. The tube was placed into a magnetic rack. Once the liquid was clear, the supernatant was discarded, and $200 \mu 1$ prewarmed stringent wash buffer were added to the beads. In an effort to avoid any temperature drop, it is important to work quickly according to the manufacturer's instructions. The beads in stringent wash buffer were incubated in a thermomixer set to $47^{\circ} \mathrm{C}$ and 1,100 rpm for $5 \mathrm{~min}$. The wash with pre-warmed stringent wash buffer was repeated once. Next, $200 \mu 1$ pre-warmed wash buffer I was added to the beads and vortexed at 1,400 rpm (room temperature) for $2 \mathrm{~min}$. After removing the respective wash buffer, beads were vortexed at 1,400 rpm in $200 \mu \mathrm{l}$ wash buffer II for $1 \mathrm{~min}$ and $200 \mu 1$ wash buffer III for $30 \mathrm{sec}$. After the final the final wash, as much liquid as possible was removed and the beads were resuspended in $40 \mu 1$ molecular-grade water.

The captured DNA was directly amplified from the beads using KAPA HiFi HotStart master mix and 25 pmol of each TruSeq PCR primer in two separate 50- $\mu$ l PCR reactions. The PCR conditions were the same as outlined above except that 14 cycles were used for amplification. The PCR reactions were pooled and the DNA was purified using $100 \mu$ AMPure XP beads. The DNA was eluted with $11 \mu 1$ elution buffer. The eluted DNA was subjected to another round of probe-mediated capture with the hybridization timespan reduced to $24 \mathrm{~h}$. Furthermore, after washing the beads, the captured fragments were amplified using only 10 cycles of PCR.

Post-sequencing analysis of next-generation capture-C data. The analysis was carried out in accordance with the original description of the method ${ }^{89}$ with some modifications. Paired-end reads were processed using trim_galore v0.4.2 from the Babraham Institute (UK) to trim off low-quality bases (default Phred score of 20, i.e. error probability was 0.01) and adapter contamination from the 3 ' end. Only complete mate pairs were processed further to reconstruct single reads from overlapping paired-end sequences using FLASH v1.2.1 $1^{53}$ with interleaved output settings for non-extended reads (flash --interleaved-output --max-overlap 150). FLASH reads were split in silico at DpnII restriction sites using a designated perl script (https://github.com/telenius/captureC/releases) before aligning them to the X. tropicalis genome assembly 7.1 using Bowtie2 v2.2.970 . The alignment was run with default settings and one thread only to keep the order of the reads. The view function of samtools v1.3.18 ${ }^{78}$ was used to retain alignments with a quality score of $\geq 10$. The alignments were analyzed further using a suite of perl scripts (https://github.com/Hughes-Genome-Group/captureC/releases) modified to process both chromosome and scaffold coordinates. Viewpoint coordinates included a 1-kb proximity exclusion range. Restriction fragments were classified as capture, proximity-exclusion or reporter. PCR duplicates were removed. The interaction map was based on the number of unique pairedend reads per restriction fragment. Windows of $2 \mathrm{~kb}$ incrementing by $200 \mathrm{bp}$ were used to consolidate interactions, which were normalized to 10,000 interactions per viewpoint. Promoter contacts are listed in Supplementary Table 12.

Analysis of genomic profiles, DNA motifs and super-enhancers. Unless otherwise stated, Spearman correlation $\left(\mathrm{R}_{\mathrm{s}}\right)$, relative tag density (meta-plots), principal component analysis (PCAs) and enrichment of DNA motifs were determined using a limited number of detected peaks per chromatin profile: top 2,000 peaks (Figs. 3c-g and 4c and Supplementary Figs. 5, 6 and 7d), top 5,000 peaks (Supplementary Fig. 1b) and top 10,000 peaks (Figs. 4b,e and 5c and Supplementary Fig. 7a-c). $R_{s}$ and PCA were calculated using DiffBind v2.4. $8^{90}$ in R (Figs. 1d, 3d,e and 4b and Supplementary Figs. 5a, 7a,b and 12a). Z-scores of motif enrichments at RNAPII peaks were calculated using ISMARA v1.2.2 ${ }^{91}$ (https://ismara.unibas.ch/fcgi/mara) in expert mode (Fig. 1d). Heat maps of relative tag densities were hierarchically clustered (Ward's method) using the R package seriation ${ }^{92}$ except for 
those in Figs. 4c and 8d, where genomic coordinates were sorted upon significant changes in DNA occupancy or chromatin accessibility (see Figure legends). In Supplementary Fig. 6, cluster leaves were additionally ordered with the Gruvaeus-Wainer's algorithm. The matrices of normalized tag densities, p-values and motif instances were generated with HOMER as follows: annotatePeaks.pl -size 2000 (or 200 for Supplementary Fig. 6) -hist 25 (bin size) -ghist. In heat maps, all genomic profiles were scaled to the means of the highest bin values of the top 100 (or 1,000 in Figs. 4c and 8d) peaks. In addition, to visualize low RNAPII binding and RNA expression, their top means were divided by 5 or 500 , respectively (Fig. 8d). Super-enhancers were assembled by stitching together peaks that are $\leq 25 \mathrm{~kb}$ apart: findPeaks -style super -fragLength 175 -inputFragLength 175 -fdr 0.001 -gsize 1.435e9 -F 3 -L 1 -localSize 10000 -C 0.97 -superSlope 1 -minDist 25000. Subsequently, we combined these superenhancers according to the developmental stage of profiling (see Supplementary Table 6 for genomic coordinates). Superenhancers $\leq 5 \mathrm{~kb}$ from TSS apart were associated with zygotic genes defined by RNAPII-mediated transcription elongation ${ }^{52}$.

Analysis of enriched gene ontology (GO) terms. Over-represented GO terms were found by applying hypergeometric tests of the Bioconductor/R package GOstats $\mathrm{v} 2.42 .0^{93}$ on gene lists. The process was also supported by the Bioconductor/R packages GSEABase v1.38.1 $1^{94}$ and GO.db v3.4.1 ${ }^{95}$. The gene universe was associated with GO terms by means of BLAST2GO ${ }^{96}$ as previously outlined ${ }^{67,97}$.

Differential analysis of chromatin features. The significance of differential chromatin accessibility between control and $\mathrm{mPouV} / \mathrm{Sox} 3$ LOF embryos was assessed at pCRMs using the Bioconductor/R package DiffBind v2.4.8 ${ }^{90}$ and the DESeq $2^{81}$ algorithm. The size of all pCRMs was fixed to $500 \mathrm{bp}$. Only the very 5' end of each aligned DNase-Seq read, which represents the DNase cleavage site, was considered for this analysis. Read counts from mapping DNase-digested naked genomic DNA were subtracted from chromatin accessibility readings at each pCRMs. Any changes to DNase-mediated cleavages at pCRMs with FDR $\leq 10 \%$ were determined as statistically significant (Fig. 8b). For visualization, the orientations of pCRMs were aligned to the direction of transcription of the closest zygotic gene. Matrices of normalized and raw tag counts were generated at pCRMs for various chromatin features and RNA (Fig. 8b). Their rows were sorted according to the statistical significance of differential chromatin accessibility. Raw tag counts in sliding (+200 bp) windows of 400 bp (accessibility, H3K4me1, RNAPII, Smad2 and $\beta$-catenin) or $2 \mathrm{~kb}$ (RNA and promoter contacts) across the genome were processed to estimate p-values of chromatin changes $(\mathrm{p} \Delta)$ using DESeq2 v1.16.1 ${ }^{81}$. Read count dispersions were locally fitted. The shrinkage of $\log _{2}$ fold changes, threshold on Cook's distances and independent filtering were all switched off. For the comparison of local interactions with promoters at affected and unaffected pCRMs, the size of the pCRMs was widened to $1.2 \mathrm{~kb}$ and filtered for $\geq 5$ reporter fragments (Fig. 9c). Normalized line and bar graph tracks of control and mPouV/Sox3 LOF embryos were superimposed in the IGV genome browser v2.3.92 ${ }^{98}$ (Figs. 9f and 10a,b and Supplementary Figs. 13 and 14).

Software for computational analysis and graphical illustration. Post-alignment analysis and graphical illustration were performed using samtools v1.3.1 ${ }^{78}$, bedtools v2.25.099, HOMER v4.8.3 ${ }^{71}$ and tools in R v3.4.1 / Bioconductor v3.5, Perl v5.18.2 or Python v2.7.12.

Quantification and statistical analysis. No statistical method was used for determining sample size. We followed the literature to select the appropriate sample size. The experiments were not randomized. Due to the nature of experiments, the authors were not blinded to group allocation during data collection and analysis. Only viable embryos and embryonic tissues were included in the analysis. Due to space constraints, data from the trials of establishing and optimizing protocols (ChIP-Seq, DNase-Seq and next-generation capture-C) were excluded. Frequencies of shown morphological phenotypes and WMISH patterns are included in every image. Significances of normally or non-normally distributed data points were calculated using a paired Student's t-test (onetailed or two-tailed) or paired Wilcoxon test (two-tailed), respectively. The effect size $\mathrm{r}$ was estimated from the standard normal deviate of the Wilcoxon $p$-value (p) as previously described ${ }^{100}, r_{\text {effect }}=Z / \operatorname{sqrt}(N)$, where $Z=q$ norm(1-p/2) is the standardized $Z$-score and $\mathrm{N}$ is the number of observations. Significance of motif enrichments or GO terms are based on hypergeometric tests. For RNA- 
Seq, biological triplicates were used to account for transcriptional variability between clutches. Each LOF experiment has its own control embryos collected in parallel from the same mothers: exp. \#1 ( $\alpha$-amanitin), uninjected embryos; exp. \#2 (BMP or Nodal LOF), DMSO-treated embryos; exp. \#3 (Wnt LOF), uninjected embryos; exp. \#4 (mPou5f3/Sox3 LOF), uninjected embryos; exp. \#5 (mVegT LOF), uninjected embryos. The gene expressions of control MO-injected embryos of exp. \#2 and \#5 were normalized to their corresponding uninjected embryos. The mean of these normalizations and conservative FDR estimations (i.e., higher FDR of the two likelihood ratio tests) were used for the comparison with LOF conditions. RNA-Seq libraries from each experiment were generated simultaneously to mitigate any batch effects. The significance of transcriptome-wide differential expression was adjusted for multiple comparisons using the Benjamini-Hochburg procedure.

1292

External datasets. The following external datasets were used for this study: $5 \mathrm{mC}$ at stage 9, and ChIP for $\mathrm{H} 3 \mathrm{~K} 4 \mathrm{me} 3$ and H3K27me3 at stage 9 (GSE67974), $\beta$-catenin ChIP at stage $10^{+}$(GSE72657), Smad2 ChIP at stage $10^{+}$(GSE30146 and GSE53654), Foxh1 ChIP at stage 8 and $10^{+}$(GSE85273 and GSE53654), total RNA recorded at 30-min intervals from unfertilized eggs to $23.5 \mathrm{hpf}$ (GSE65785), ribosome footprinting from stage 3, 9 and 12 (GSE52809), and regional expression along the animal-vegetal and dorso-ventral axis at stage $10^{+}$(GSE81458).

Data and code availability. Programming code and some intermediate data underlying Figs. 1d-f, 2b, 3c-g, 4b,c,e, 5c, 6e,f, 7a,b,e, 8b-g and 9c, Supplementary Figs. 1a,b,d, 5a,b, 7a-d, 9, 10a,b, 11a,b, 12a and 15-17 and Supplementary Tables 9, 11 and 12 are available on GitHub at https://github.com/gegentsch/SignalCompetence.

1302

\section{REFERENCES}

1304 1. Waddington, C. H. Organisers and Genes. Cambridge University Press (1940).

1305 2. Grainger, R. M. Embryonic lens induction: shedding light on vertebrate tissue determination. Trends Genet. 8, 349-355 (1992).

1309

\section{Tada, M., O'Reilly, M. \& Smith, J. Analysis of competence and of Brachyury autoinduction by use of hormone-} inducible Xbra. Development 124, 2225-2234 (1997).

4. Fujiwara, M., Uchida, T., Osumi-Yamashita, N. \& Eto, K. Uchida rat (rSey): a new mutant rat with craniofacial abnormalities resembling those of the mouse Sey mutant. Differentiation 57, 31-38 (1994).

5. Pagliuca, F. W. et al. Generation of functional human pancreatic $\beta$ cells in vitro. Cell 159, 428-439 (2014).

6. Torres, J. et al. A switch in transcription and cell fate governs the onset of an epigenetically-deregulated tumor in Drosophila. Elife 7, e32697 (2018).

7. Briggs, J. A. et al. The dynamics of gene expression in vertebrate embryogenesis at single-cell resolution. Science eaar5780 (2018).

8. Gerstein, M. B. et al. Architecture of the human regulatory network derived from ENCODE data. Nature 489, 91-100 (2012).

9. Schohl, A. \& Fagotto, F. Beta-catenin, MAPK and Smad signaling during early Xenopus development. Development 129, 37-52 (2002).

10. Buecker, C. et al. A murine ESC-like state facilitates transgenesis and homologous recombination in human pluripotent stem cells. Cell Stem Cell 6, 535-546 (2010).

11. Buenrostro, J. D., Giresi, P. G., Zaba, L. C., Chang, H. Y. \& Greenleaf, W. J. Transposition of native chromatin for fast and sensitive epigenomic profiling of open chromatin, DNA-binding proteins and nucleosome position. Nat. Methods 10, 1213-1218 (2013).

12. Wühr, M. et al. Deep proteomics of the Xenopus laevis egg using an mRNA-derived reference database. Curr. Biol. 24, 1467-1475 (2014).

13. Subtelny, A. O., Eichhorn, S. W., Chen, G. R., Sive, H. \& Bartel, D. P. Poly(A)-tail profiling reveals an embryonic switch in translational control. Nature 508, 66-71 (2014).

14. De Domenico, E., Owens, N. D. L., Grant, I. M., Gomes-Faria, R. \& Gilchrist, M. J. Molecular asymmetry in the 8-cell stage Xenopus tropicalis embryo described by single blastomere transcript sequencing. Dev. Biol. 408, 252-268 (2015).

15. Stennard, F., Zorn, A. M., Ryan, K., Garrett, N. \& Gurdon, J. B. Differential expression of VegT and Antipodean protein isoforms in Xenopus. Mech. Dev. 86, 87-98 (1999).

16. Larabell, C. A. et al. Establishment of the dorso-ventral axis in Xenopus embryos is presaged by early asymmetries in beta-catenin that are modulated by the Wnt signaling pathway. J. Cell. Biol. 136, 1123-1136 (1997).

17. Faure, S., Lee, M. A., Keller, T., Dijke, ten, P. \& Whitman, M. Endogenous patterns of TGFbeta superfamily signaling during early Xenopus development. Development 127, 2917-2931 (2000).

18. Charney, R. M. et al. Foxh1 Occupies cis-Regulatory Modules Prior to Dynamic Transcription Factor Interactions Controlling the Mesendoderm Gene Program. Dev. Cell 40, 595-607.e4 (2017). 
19. Gentsch, G. E. et al. In vivo T-box transcription factor profiling reveals joint regulation of embryonic neuromesodermal bipotency. Cell Rep. 4, 1185-1196 (2013).

20. Gentsch, G. E., Monteiro, R. S. \& Smith, J. C. Cooperation Between T-Box Factors Regulates the Continuous Segregation of Germ Layers During Vertebrate Embryogenesis. Curr. Top. Dev. Biol. 122, 117-159 (2017).

21. Whyte, W. A. et al. Master transcription factors and mediator establish super-enhancers at key cell identity genes. Cell 153, 307-319 (2013).

22. Müller, C. W. \& Herrmann, B. G. Crystallographic structure of the T domain-DNA complex of the Brachyury transcription factor. Nature 389, 884-888 (1997).

23. Chen, X., Rubock, M. J. \& Whitman, M. A transcriptional partner for MAD proteins in TGF-beta signalling. Nature 383, 691-696 (1996).

24. Chen, X. et al. Integration of external signaling pathways with the core transcriptional network in embryonic stem cells. Cell 133, 1106-1117 (2008).

25. Chiu, W. T. et al. Genome-wide view of TGF /Foxh1 regulation of the early mesendoderm program. Development 141, 1-114 (2014).

26. Heasman, J., Kofron, M. \& Wylie, C. $\beta$ Catenin Signaling Activity Dissected in the Early Xenopus Embryo: A Novel Antisense Approach. Dev. Biol. 222, 124-134 (2000).

27. Gentsch, G. E. et al. Innate Immune Response and Off-Target Mis-splicing Are Common Morpholino-Induced Side Effects in Xenopus. Dev. Cell 44, 597-610.e10 (2018).

28. Cuny, G. D. et al. Structure-activity relationship study of bone morphogenetic protein (BMP) signaling inhibitors. Bioorg. Med. Chem. Lett. 18, 4388-4392 (2008).

29. Young, J. J. et al. Noggin is required for first pharyngeal arch differentiation in the frog Xenopus tropicalis. Dev. Biol. 426, 245-254 (2017).

30. Ho, D. M., Chan, J., Bayliss, P. \& Whitman, M. Inhibitor-resistant type I receptors reveal specific requirements for TGF-beta signaling in vivo. Dev. Biol. 295, 730-742 (2006).

31. Inman, G. J. et al. SB-431542 is a potent and specific inhibitor of transforming growth factor-beta superfamily type I activin receptor-like kinase (ALK) receptors ALK4, ALK5, and ALK7. Mol. Pharmacol. 62, 65-74 (2002). Skirkanich, J., Luxardi, G., Yang, J., Kodjabachian, L. \& Klein, P. S. An essential role for transcription before the MBT in Xenopus laevis. Dev. Biol. 357, 478-491 (2011).

33. Reversade, B., Kuroda, H., Lee, H., Mays, A. \& De Robertis, E. M. Depletion of Bmp2, Bmp4, Bmp7 and Spemann organizer signals induces massive brain formation in Xenopus embryos. Development 132, 3381-3392 (2005).

34. Jacobs, J. et al. The transcription factor Grainy head primes epithelial enhancers for spatiotemporal activation by displacing nucleosomes. Nat. Genet. 50, 1011-1020 (2018).

35. Mullen, A. C. et al. Master Transcription Factors Determine Cell-Type-Specific Responses to TGF- $\beta$ Signaling. Cell 147, 565-576 (2011).

36. Trompouki, E. et al. Lineage regulators direct BMP and Wnt pathways to cell-specific programs during differentiation and regeneration. Cell 147, 577-589 (2011).

37. Soufi, A. et al. Pioneer transcription factors target partial DNA motifs on nucleosomes to initiate reprogramming. Cell 161, 555-568 (2015).

38. Oosterveen, T. et al. Mechanistic differences in the transcriptional interpretation of local and long-range Shh morphogen signaling. Dev. Cell 23, 1006-1019 (2012).

39. Peshkin, L. et al. On the Relationship of Protein and mRNA Dynamics in Vertebrate Embryonic Development. Dev. Cell 35, 383-394 (2015).

40. Peshkin, L. et al. The protein repertoire in early vertebrate embryogenesis. bioRxiv 1865, 571174 (2019).

41. Gurdon, J. B. Cell Fate Determination by Transcription Factors. Curr. Top. Dev. Biol. 116, 445-454 (2016).

42. Neph, S. et al. An expansive human regulatory lexicon encoded in transcription factor footprints. Nature 489, 8390 (2012).

43. Tapia, N. et al. Dissecting the role of distinct OCT4-SOX2 heterodimer configurations in pluripotency. Sci. Rep. 5, 13533 (2015).

44. Lee, M. T. et al. Nanog, Pou5f1 and SoxB1 activate zygotic gene expression during the maternal-to-zygotic transition. Nature 503, 360-364 (2013).

45. Leichsenring, M., Maes, J., Mössner, R., Driever, W. \& Onichtchouk, D. Pou5f1 transcription factor controls zygotic gene activation in vertebrates. Science 341, 1005-1009 (2013).

46. Scerbo, P. et al. Ventx Factors Function as Nanog-Like Guardians of Developmental Potential in Xenopus. PLoS ONE 7, e36855 (2012).

47. Iwafuchi-Doi, M. \& Zaret, K. S. Cell fate control by pioneer transcription factors. Development 143, 1833-1837 (2016).

48. Zhang, C., Basta, T., Jensen, E. D. \& Klymkowsky, M. W. The beta-catenin/VegT-regulated early zygotic gene Xnr5 is a direct target of SOX3 regulation. Development 130, 5609-5624 (2003).

49. Beck, C. W. \& Slack, J. M. Analysis of the developing Xenopus tail bud reveals separate phases of gene expression during determination and outgrowth. Mech. Dev. 72, 41-52 (1998).

50. Owens, N. D. L. et al. Measuring Absolute RNA Copy Numbers at High Temporal Resolution Reveals Transcriptome Kinetics in Development. Cell Rep. 14, 632-647 (2016).

51. Blitz, I. L. et al. A catalog of Xenopus tropicalis transcription factors and their regional expression in the early gastrula stage embryo. Dev. Biol. 426, 409-417 (2017).

52. Gentsch, G. E., Owens, N. D. L. \& Smith, J. C. The Spatio-Temporal Control of Zygotic Genome Activation. bioRxiv 488056 (2018). 
53. Magoč, T. \& Salzberg, S. L. FLASH: fast length adjustment of short reads to improve genome assemblies. Bioinformatics 27, 2957-2963 (2011).

54. Hontelez, S. et al. Embryonic transcription is controlled by maternally defined chromatin state. Nat. Commun. 6, 10148 (2015).

55. Khokha, M. et al. Techniques and probes for the study of Xenopus tropicalis development. Dev. Dyn. 225, 499510 (2002).

56. Sive, H., Grainger, R. \& Harland, R. Early development of Xenopus laevis: A laboratory manual. Cold Spring Harbor Laboratory Press (2000).

57. Nieuwkoop, P. \& Faber, J. Normal table of Xenopus laevis (Daudin): a systematical and chronological survey of the development from the fertilized egg till the end of metamorphosis. Garland (1994).

58. Rana, A., Collart, C., Gilchrist, M. \& Smith, J. Defining synphenotype groups in Xenopus tropicalis by use of antisense morpholino oligonucleotides. PLoS Genet. 2, e193 (2006).

59. Hopwood, N., Pluck, A. \& Gurdon, J. MyoD expression in the forming somites is an early response to mesoderm induction in Xenopus embryos. Embo J. 8, 3409-3417 (1989).

60. Gökirmak, T. et al. Localization and substrate selectivity of sea urchin multidrug (MDR) efflux transporters. $J$. Biol. Chem. 287, 43876-43883 (2012).

61. Ladher, R., Mohun, T. J., Smith, J. C. \& Snape, A. M. Xom: a Xenopus homeobox gene that mediates the early effects of BMP-4. Development 122, 2385-2394 (1996).

62. Smith, J., Price, B., Green, J., Weigel, D. \& Herrmann, B. Expression of a Xenopus homolog of Brachyury (T) is an immediate-early response to mesoderm induction. Cell 67, 79-87 (1991).

63. Monsoro-Burq, A. H. A Rapid Protocol for Whole-Mount In Situ Hybridization on Xenopus Embryos. Cold Spring Harb. Protoc. 2007, pdb.prot4809 (2007).

64. Landt, S. G. et al. ChIP-seq guidelines and practices of the ENCODE and modENCODE consortia. Genome Res. 22, 1813-1831 (2012).

65. Gentsch, G. E. \& Smith, J. C. Efficient Preparation of High-Complexity ChIP-Seq Profiles from Early Xenopus Embryos. Methods Mol. Biol. 1507, 23-42 (2017).

66. Livak, K. J. \& Schmittgen, T. D. Analysis of Relative Gene Expression Data Using Real-Time Quantitative PCR and the 2CT Method. Methods 25, 402-408 (2001).

67. Gentsch, G. E., Patrushev, I. \& Smith, J. C. Genome-wide snapshot of chromatin regulators and states in Xenopus embryos by ChIP-Seq. J. Vis. Exp. e52535 (2015).

68. Gentsch, G. E. \& Smith, J. C. Investigating physical chromatin associations across the Xenopus genome by chromatin immunoprecipitation. Cold Spring Harb. Protoc. 2014, pdb.prot080614 (2014).

69. Gentsch, G. E. \& Smith, J. C. Mapping Chromatin Features of Xenopus Embryos. Cold Spring Harb. Protoc. 2019, pdb.prot100263 (2019). Langmead, B. \& Salzberg, S. L. Fast gapped-read alignment with Bowtie 2. Nat. Methods 9, 357-359 (2012). Heinz, S. et al. Simple combinations of lineage-determining transcription factors prime cis-regulatory elements required for macrophage and B cell identities. Mol. Cell 38, 576-589 (2010).

Nakamura, Y., de Paiva Alves, E., Veenstra, G. J. C. \& Hoppler, S. Tissue- and stage-specific Wnt target gene expression is controlled subsequent to $\beta$-catenin recruitment to cis-regulatory modules. Development 143, 19141925 (2016).

Yoon, S.-J., Wills, A. E., Chuong, E., Gupta, R. \& Baker, J. C. HEB and E2A function as SMAD/FOXH1 cofactors. Genes Dev. 25, 1654-1661 (2011).

Smit, A., Hubley, R. \& Green, P. RepeatMasker Open-4.0. 2013-2015.

Benson, G. Tandem repeats finder: a program to analyze DNA sequences. Nucleic Acids Res. 27, 573-580 (1999).

Karpinka, J. B. et al. Xenbase, the Xenopus model organism database; new virtualized system, data types and genomes. Nucleic Acids Res. 43, D756-63 (2015).

Dobin, A. et al. STAR: ultrafast universal RNA-seq aligner. Bioinformatics 29, 15-21 (2013).

Li, H. et al. The Sequence Alignment/Map format and SAMtools. Bioinformatics 25, 2078-2079 (2009). Zhu, Q., Fisher, S. A., Shallcross, J. \& Kim, J. VERSE: a versatile and efficient RNA-Seq read counting tool. bioRxiv 053306 (2016). DESeq2. Genome Biol. 15, 550 (2014).

82. Quast, C. et al. The SILVA ribosomal RNA gene database project: improved data processing and web-based tools. Nucleic Acids Res. 41, D590-6 (2013).

83. Langmead, B., Trapnell, C., Pop, M. \& Salzberg, S. L. Ultrafast and memory-efficient alignment of short DNA sequences to the human genome. Genome Biol. 10, R25 (2009).

84. Kim, D. et al. TopHat2: accurate alignment of transcriptomes in the presence of insertions, deletions and gene fusions. Genome Biol. 14, R36 (2013).

85. Song, L. \& Crawford, G. E. DNase-seq: a high-resolution technique for mapping active gene regulatory elements across the genome from mammalian cells. Cold Spring Harb. Protoc. 2010, pdb.prot5384 (2010).

86. He, H. H. et al. Refined DNase-seq protocol and data analysis reveals intrinsic bias in transcription factor footprint identification. Nat. Methods 11, 73-78 (2014).

87. Vierstra, J., Wang, H., John, S., Sandstrom, R. \& Stamatoyannopoulos, J. A. Coupling transcription factor occupancy to nucleosome architecture with DNase-FLASH. Nat. Methods 11, 66-72 (2014). 
bioRxiv preprint doi: https://doi.org/10.1101/306803; this version posted June 3, 2019. The copyright holder for this preprint (which was not certified by peer review) is the author/funder, who has granted bioRxiv a license to display the preprint in perpetuity. It is made available under aCC-BY 4.0 International license.

88. Kohany, O., Gentles, A. J., Hankus, L. \& Jurka, J. Annotation, submission and screening of repetitive elements in Repbase: RepbaseSubmitter and Censor. BMC Bioinformatics 7, 474 (2006).

89. Davies, J. O. J. et al. Multiplexed analysis of chromosome conformation at vastly improved sensitivity. Nat. Methods 13, 74-80 (2016).

90. $\quad$ Stark, R. \& Brown, G. DiffBind: Differential binding analysis of ChIP-Seq peak data. 1-33 (2018).

91. Balwierz, P. J. et al. ISMARA: automated modeling of genomic signals as a democracy of regulatory motifs. Genome Res. 24, 869-884 (2014).

92. Hahsler, M., Hornik, K. \& Buchta, C. Getting Things in Order: An Introduction to the R Package seriation. $J$. Stat. Softw. 25 (2008).

93. Falcon, S. \& Gentleman, R. Using GOstats to test gene lists for GO term association. Bioinformatics 23, 257-258 (2007).

94. Morgan, M., Falcon, S. \& Gentleman, R. GSEABase: Gene set enrichment data structures and methods. (2017).

95. Carlson, M., Falcon, S., Pages, H. \& Li, N. Bioconductor - GO.db. (2007).

96. Conesa, A. et al. Blast2GO: a universal tool for annotation, visualization and analysis in functional genomics research. Bioinformatics 21, 3674-3676 (2005).

97. Collart, C. et al. High-resolution analysis of gene activity during the Xenopus mid-blastula transition. Development 141, 1927-1939 (2014).

98. Robinson, J. T. et al. Integrative genomics viewer. Nat. Biotechnol. 29, 24-26 (2011).

99. Quinlan, A. R. \& Hall, I. M. BEDTools: a flexible suite of utilities for comparing genomic features. Bioinformatics 26, 841-842 (2010).

100. Rosenthal, R. Meta-Analytic Procedures for Social Research. SAGE Publishing (1991). 